\title{
Tauberian-Cardy formula with spin
}

\section{Sridip Pal ${ }^{a, b}$ and Zhengdi Sun ${ }^{a}$}

${ }^{a}$ Department of Physics, University of California San Diego, 9500 Gilman Drive, La Jolla, CA 92093, U.S.A.

${ }^{b}$ School of Natural Sciences, Institute for Advanced Study, One Einstein Drive, Princeton, NJ 08540, U.S.A.

E-mail: sridip@ias.edu, z5sun@ucsd.edu

ABSTRACT: We prove a 2 dimensional Tauberian theorem in context of 2 dimensional conformal field theory. The asymptotic density of states with conformal weight $(h, \bar{h}) \rightarrow$ $(\infty, \infty)$ for any arbitrary spin is derived using the theorem. We further rigorously show that the error term is controlled by the twist parameter and insensitive to spin. The sensitivity of the leading piece towards spin is discussed. We identify a universal piece in microcanonical entropy when the averaging window is large. An asymptotic spectral gap on $(h, \bar{h})$ plane, hence the asymptotic twist gap is derived. We prove an universal inequality stating that in a compact unitary 2D CFT without any conserved current $A g \leq \frac{\pi(c-1) r^{2}}{24}$ is satisfied, where $g$ is the twist gap over vacuum and $A$ is the minimal "areal gap", generalizing the minimal gap in dimension to $\left(h^{\prime}, \bar{h}^{\prime}\right)$ plane and $r=\frac{4 \sqrt{3}}{\pi} \simeq 2.21$. We investigate density of states in the regime where spin is parametrically larger than twist with both going to infinity. Moreover, the large central charge regime is studied. We also probe finite twist, large spin behavior of density of states.

Keywords: Conformal and W Symmetry, Conformal Field Theory

ArXiv EPRINT: 1910.07727 


\section{Contents}

1 Summary \& discussion $\quad 1$

1.1 Integrated density of states 3

1.1.1 Main theorems on integrated density of states 3

1.1.2 Corollaries of the theorems [eq. (1.1) and eq. (1.2)] on integrated density of states 4

$1.2 \quad c>1$ CFTs-results specific for primaries $\quad 7$

1.3 Large spin, finite twist sector 8

$\begin{array}{lll}1.4 & \text { Asymptotic spectral gap } & 10\end{array}$

$\begin{array}{lll}\text { 1.4.1 Probing spectral gap via circle of order one area } & 10\end{array}$

$\begin{array}{lll}\text { 1.4.2 Probing spectral gap via strips } & 14\end{array}$

$\begin{array}{ll}1.5 & \text { Analysis at large central charge } \\ \end{array}$

2 Set up 16

$\begin{array}{lll}2.1 & \text { A semi technical glimpse of the subtleties } & 16\end{array}$

$3 \quad O(1)$ rectangular window $\quad 19$

$\begin{array}{ll}3.1 \text { Generic analysis: with/without twist gap } & 19\end{array}$

3.2 Analysis of $O(1)$ window assuming a twist gap 24

4 Lemma: density of states on strip of order one width 28

5 The integrated density of states $\quad 32$

5.1 The main 2D Tauberian theorem 32

$\begin{array}{lll}5.2 & \text { Sensitivity of asymptotics towards spin } J & 38\end{array}$

6 Verification: 2D Ising model $\quad 39$

$\begin{array}{lll}7 & \text { Finite twist-large spin } & 41\end{array}$

8 Holographic CFTs $\quad 45$

$9 \begin{array}{ll}9 & \text { Open problems }\end{array}$

\section{Summary \& discussion}

The Cardy formula [1] for the asymptotic density of states has recently been rigorously derived with an estimate for the error term in $[2,3]$. A natural question is to ask whether one can generalize the formalism so as to make it sensitive to the spin or equivalently to the 
conformal weights $h, \bar{h}$ separately. This necessitates working out a 2 dimensional Tauberian theorem, which we achieve here. The motivations for investigating Cardy formula on $\left(h^{\prime}, \bar{h}^{\prime}\right)$ plane are several. First of all, the notion of infinity on a $2 \mathrm{~d}$ plane is richer than $\Delta \rightarrow \infty$ limit. We will see that the finer details of the Cardy formula actually depend on how infinity is approached unless one makes extra assumption about the spectrum. Furthermore, there have been interesting developments in the direction of lightcone bootstrap in recent times [4-8], our analysis puts some of these results on rigorous footing. Another amazing feature is the ability to investigate the "areal" notion of spectral gap. If we probe the $\left(h^{\prime}, \bar{h}^{\prime}\right)$ plane with circular areas of radius $R$, centered at $(h, \bar{h})$, then we find the optimal value of $R$ which guarantees that the area contains at least one state. Again unless we put in extra assumption, the value of $R$ depends on how infinity is approached and thus showing a richer asymptotic behavior. If we one assumes existence of twist gap, it turns out that the twist gap is complementary to asymptotic spectral gap in some sense, which we will make precise in due course.

The naive Cardy like analysis provides us with an expression for the asymptotic density of states where $h$ and $\bar{h}$ are of the same order. One can re-express this as a function of dimension $\Delta$ and spin $J$ with $\Delta \simeq J$. Now a natural question is to ask whether the result is valid when $\Delta$ and $J$ is not of the same order. For example, in the large charge expansion literature [9-18], the regime where $J \simeq \Delta^{1 / n}$ with $n>1$ is being probed. It turns out that only a part of the answer coming from the naive Cardy like analysis is meaningful while the rest of it is comparable to the error term. We emphasize that the analysis is only possible because now we have a rigorous estimate of the error term due to the Tauberian theorem that we prove in this paper.

With our rigorous treatment, it is possible to address issues regarding whether we can trust the naive Cardy formula when $h$ and $\bar{h}$ are not of the same order. It turns out that the answer to this question is intimately connected with the existence of twist gap. We show that we can trust the naive Cardy formula for all the operators when $\max (h, \bar{h})=\min (h, \bar{h})^{\Upsilon}$ with $1 \leq \Upsilon<2$. It is also shown that with the assumption of twist gap, the validity of Cardy formula for primaries for $c>1$ CFTs does not require any restriction on $\Upsilon$.

The another motivation for taking up a rigorous study of Cardy formula is to be able to probe the large central charge $(c)$ sector, to be specific, to derive the density of states when $h / c, \bar{h} / c$ are finite but $c$ is very large. This part is in the spirit of result derived in [19]. A nice feature that reveals itself through the rigorous treatment is a curious connection between validity of Cardy regime and the twist gap above the vacuum. These features are important in the context of holography.

The plan of the paper is to quote the main results here in the beginning and discuss its consequences in terms of CFT data, such that the current section can be thought of as mostly self contained. The next section 2 gives some intuitive understanding of the technical stuff that follows. From section 3 onwards, we plunge into technical proofs with a healthy relaxing intermission in section 6 , where we numerically verify our results. For readers going for a really quick ride, we have highlighted the main equations and results in what follows. 


\subsection{Integrated density of states}

We prove a 2 dimensional Tauberian theorem in context of 2 dimensional conformal field theory. The asymptotic density of states with conformal weight $(h, \bar{h}) \rightarrow(\infty, \infty)$ is derived using the theorem. We find that the error term is controlled by the twist parameter. We note that as $(h, \bar{h}) \rightarrow(\infty, \infty)$, the twist also goes to $\infty$. We remark that the regime of validity depends on whether we put in the assumption of having a twist gap.

Definition 1.1. By finite twist gap, we mean there exists a number $\tau_{*}>0$ such that there is no operator with twist $\tau \in\left(0, \tau_{*}\right)$ and there are finite number of zero twist operators ${ }^{1}$ with dimension less than $c / 12$.

We make two remarks: a) the fact that there are finite number of zero twist operators with dimension less than $c / 12$ is always true since there are finite number of operators with dimension less than $c / 12$ for finite central charge, b) Not having any operator with twist $\tau \in\left(0, \tau_{*}\right)$ disallows having 0 as twist accumulation point.

\subsubsection{Main theorems on integrated density of states}

No assumption on twist gap. We show that for finite central charge $c$, the number of states with conformal weights less than or equal to some specified large conformal weight $h, \bar{h}$ is given by:

$$
\begin{aligned}
& F(h, \bar{h}) \equiv \int_{0}^{h} \mathrm{~d} h^{\prime} \int_{0}^{\bar{h}} \mathrm{~d} \bar{h}^{\prime} \rho\left(h^{\prime}, \bar{h}^{\prime}\right) \\
& \underset{\substack{h / \bar{h}=O(1) \\
h, \bar{h} \rightarrow \infty}}{=} \frac{1}{4 \pi^{2}}\left(\frac{36}{c^{2} h \bar{h}}\right)^{1 / 4} \exp \left[2 \pi\left(\sqrt{\frac{c h}{6}}+\sqrt{\frac{c \bar{h}}{6}}\right)\right]\left[1+O\left(\tau^{-1 / 4}\right)\right],
\end{aligned}
$$

where $\tau$ is the twist of the state with $h, \bar{h}$ and given by $\tau=2 \min \{h, \bar{h}\}$. Here we have assumed that $h / \bar{h}=O(1)$ number. $^{2}$ As a result one could have written the error term as $O\left(h^{-1 / 4}\right)$ or $O\left(\bar{h}^{-1 / 4}\right)$.

Assuming a twist gap. It turns out that if we assume a finite twist gap, we can trust eq. (1.1) even when $h$ and $\bar{h}$ are not of the same order but $h=\bar{h}^{v}$ with $1 / 2<v<2$. In such a scenario, the error term becomes $O\left(\tau^{\frac{\Upsilon}{4}-1 / 2}\right)$, where $\Upsilon=\max (v, 1 / v)$. The $\Upsilon$ characterizes how $h$ and $\bar{h}$ are of different order asymptotically in a symmetrized fashion, for example, if we approach the infinity along the curve $h=\bar{h}^{1.1}$ or $\bar{h}=h^{1.1}$, we have $\Upsilon=1.1$. Thus our error estimation is symmetric if we reflect the line of approach to infinity about $h=\bar{h}$ line.

\footnotetext{
${ }^{1}$ Usually, by finite twist gap, it is assumed that there is no zero twist primaries except the Identity. Here we are using it in a slightly different manner, so one needs to be careful about using bounds on twist gap, such as the one appearing in [20].

${ }^{2}$ If we say $f=O(1)$, we mean $|f|<M$ for a fixed positive number $M$.
} 
We have for $1 \leq \Upsilon<2$,

$$
\begin{aligned}
& F(h, \bar{h}) \equiv \int_{0}^{h} \mathrm{~d} h^{\prime} \int_{0}^{\bar{h}} \mathrm{~d} \bar{h}^{\prime} \rho\left(h^{\prime}, \bar{h}^{\prime}\right) \\
& \underset{\substack{h, \bar{h} \rightarrow \infty \\
\frac{1}{2}<\frac{\log h}{\log h<2}}}{=} \frac{1}{4 \pi^{2}}\left(\frac{36}{c^{2} h \bar{h}}\right)^{1 / 4} \exp \left[2 \pi\left(\sqrt{\frac{c h}{6}}+\sqrt{\frac{c \bar{h}}{6}}\right)\right]\left[1+O\left(\tau^{\frac{\Upsilon}{4}-1 / 2}\right)\right], \quad \Upsilon<2 .
\end{aligned}
$$

The eq. (1.1) and eq. (1.2) are two of the central results obtained in this paper. If $h$ and $\bar{h}$ are not of the same order, we basically probe the large spin sector of density of states, ${ }^{3}$ to be precise, the regime where spin is parametrically larger than the twist but both goes to infinity.

The basic structure of both the eq. (1.1) and eq. (1.2) is that they have leading exponential piece multiplied with a subleading polynomial suppression. The error term is then further suppressed by a polynomial piece. Now if $\Upsilon \geq 2$, one can see the error term in (1.2) is not really suppressed, hence is not in fact an error term. Thus we can not trust the polynomially suppressed terms. In this regime, we are able to show that

$$
F(h, \bar{h}) \underset{h, \bar{h} \rightarrow \infty}{=} \exp \left[2 \pi \sqrt{\frac{c h}{6}}+2 \pi \sqrt{\frac{c \bar{h}}{6}}\right] O\left(\tau^{-3 / 4}\right), \quad \Upsilon \geq 2 .
$$

We further remark that for CFTs where the partition function nicely factorizes into holomorphic and antiholomorphic pieces, the leading result directly follows from the analogous result for large $\Delta=h+\bar{h}$, proven in [2], nonetheless the error term in analogues of eq. (1.1) and eq. (1.2) goes like $O\left(h^{-1 / 2}\right)$, hence, in such a case, we have more control over the approximation.

\subsubsection{Corollaries of the theorems [eq. (1.1) and eq. (1.2)] on integrated density of states}

Below we will digress a bit and touch upon some of the interesting results that can be extracted from the above before coming back to summazing our main results in the next subsection 1.2.

Rich structure of asymptotic approach. The integrated density of states show distinct leading behavior depending on how the asymptotic infinity is approached. In [2], it

\footnotetext{
${ }^{3} \mathrm{~A}$ cautionary remark is that here in this paper unless otherwise mentioned, the twist is NOT kept finite while taking this limit. This can be contrasted to the scenario in the usual large spin expansion [21], where one keeps the twist finite.
} 
has been shown that as $\Delta \rightarrow \infty$, we have

$$
\begin{aligned}
F^{\mathrm{MZ}}(\Delta) & \equiv \int_{0}^{\Delta} \mathrm{d} \Delta^{\prime} \rho\left(\Delta^{\prime}\right) \\
& =\frac{1}{\Delta \rightarrow \infty}\left(\frac{3}{c \Delta}\right)^{1 / 4} \exp \left[2 \pi \sqrt{\frac{c \Delta}{3}}\right]\left[1+O\left(\Delta^{-1 / 2}\right)\right]
\end{aligned}
$$

We remark that in the asymptotic limit, both $F^{\mathrm{MZ}}(\Delta \rightarrow \infty)$ and $F(h \rightarrow \infty, \bar{h} \rightarrow \infty)$ count the total number of operators. But these functions approach infinity in a different manner (see the figure 1). To be concrete, let us choose $h=\bar{h}=\Delta / 2$, thus we have

$$
F(\Delta / 2, \Delta / 2) \underset{\Delta \rightarrow \infty}{=} \frac{1}{2 \pi^{2}}\left(\frac{3}{c \Delta}\right)^{1 / 2} \exp \left[2 \pi \sqrt{\frac{c \Delta}{3}}\right]\left[1+O\left(\Delta^{-1 / 4}\right)\right] .
$$

So we can see that $\lim _{\Delta \rightarrow \infty} F(\Delta / 2, \Delta / 2)$ is power law suppressed compared to $\lim _{\Delta \rightarrow \infty} F^{\mathrm{MZ}}(\Delta)$ i.e.

$$
\lim _{\Delta \rightarrow \infty}\left(\frac{F(\Delta / 2, \Delta / 2)}{F^{\mathrm{MZ}}(\Delta)}\right)=O\left(\Delta^{-1 / 4}\right)
$$

We see that the square $\mathbb{S}$ ı of size $\Delta / 2$ with one vertex at origin and another one at $(\Delta / 2, \Delta / 2)$ is always contained within the rightangled triangular region $\mathbb{T}$, created by $h^{\prime}$ axis, $\bar{h}^{\prime}$ axis and $h^{\prime}+\bar{h}^{\prime}=\Delta$ line. This is consistent with the observation that leading behavior of $F(\Delta / 2, \Delta / 2)$ is suppressed compared to $F^{\mathrm{MZ}}(\Delta)$. In fact, one can similarly study the distribution of the operators in rectangular (or square) areas such that the rectangle is contained within $\mathbb{T}$, and one vertex is on the line $h^{\prime}+\bar{h}^{\prime}=\Delta$ (see the figure 2). This study reveals that the among such areas, the square $\mathbb{S}$ । contains the most number of operators while any other rectangular region contains fewer number of operators, in fact the number is exponentially suppressed compared to that of the square $\mathbb{S}$ II.

Spin sensitivity of the asymptotics. One can make a detailed analysis of spin sensitivity of the above result, which we expound on section 5.2.

Windowed entropy with respect to $\boldsymbol{h}$ and $\overline{\boldsymbol{h}}$. An immediate consequence of the eq. (1.1) is the expression for "windowed" entropy $S_{\delta, \bar{\delta}}$. The windowed entropy is defined as logarithm of number of states within a rectangular window of side length $2 \delta$ and $2 \bar{\delta}$, centered at $(h, \bar{h})$. This is analogous to entropy defined as in microcanonical ensemble by proper "binning", where the bin size is dictated by $\delta, \bar{\delta}$. As we take $h \rightarrow \infty, \bar{h} \rightarrow \infty$, we can keep the bin size $\delta, \bar{\delta}$ order one or let them scale like $h^{\alpha}$ and $\bar{h}^{\alpha}$ respectively. We find that

$$
\begin{aligned}
S_{\delta, \bar{\delta}} & \equiv \log \left(\int_{h-\delta}^{h+\delta} \mathrm{d} h^{\prime} \int_{\bar{h}-\bar{\delta}}^{\bar{h}+\bar{\delta}} \mathrm{d} \bar{h}^{\prime} \rho\left(h^{\prime}, \bar{h}^{\prime}\right)\right) \\
& =2 \pi\left(\sqrt{\frac{c h}{6}}+\sqrt{\frac{c \bar{h}}{6}}\right)+\frac{1}{4} \log \left[\frac{c^{2} \delta^{4} \bar{\delta}^{4}}{36 h^{3} \bar{h}^{3}}\right]+s(\delta, \bar{\delta}, h, \bar{h}),
\end{aligned}
$$




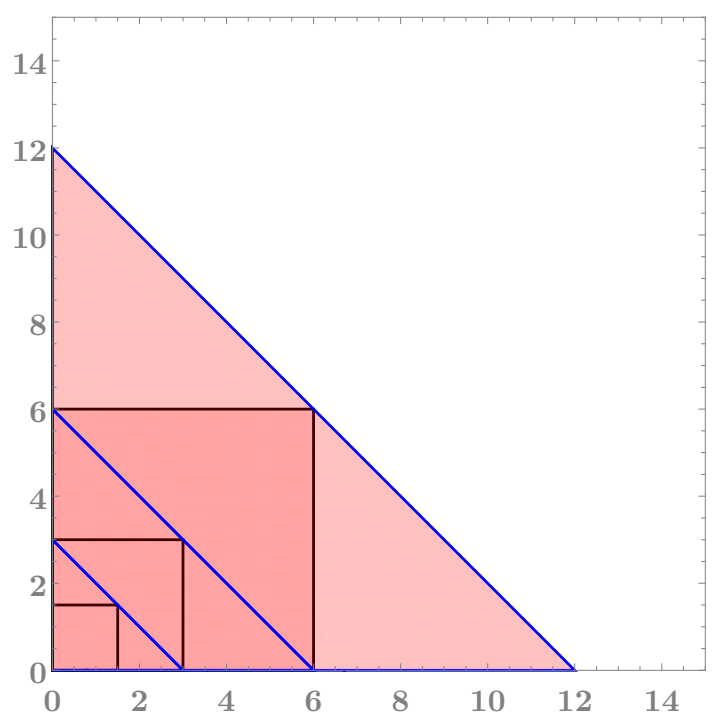

Figure 1. Approaching to infinity on $\left(h^{\prime}, \bar{h}^{\prime}\right)$ plane: the blue lines denote how the number of operators with $\left(h^{\prime}, \bar{h}^{\prime}\right)$ is counted such that $h^{\prime}+\bar{h}^{\prime} \leq \Delta$ and then we let $\Delta \rightarrow \infty$, this is given by the function $F^{\mathrm{MZ}}(\Delta)$, originally calculated in [2]. On the other hand, the black lines denote how the number of operator is counted upto some value of $h=\bar{h}=\Delta / 2$ i.e. $h^{\prime} \leq \Delta / 2, \bar{h}^{\prime} \leq \Delta / 2$ and then we let $\Delta \rightarrow \infty$. This approach to infinity is captured by the function $F(\Delta / 2, \Delta / 2)$, calculated in this paper. We see that the square of size $\Delta / 2$ with one vertex at origin and another one at $(\Delta / 2, \Delta / 2)$ are always contained within the rightangled triangular region, created by $h^{\prime}$ axis, $\bar{h}^{\prime}$ axis and $h^{\prime}+\bar{h}^{\prime}=\Delta$ line. This is consistent with the observation that leading behavior of $F(\Delta / 2, \Delta / 2)$ is suppressed compared to $F^{\mathrm{MZ}}(\Delta)$.

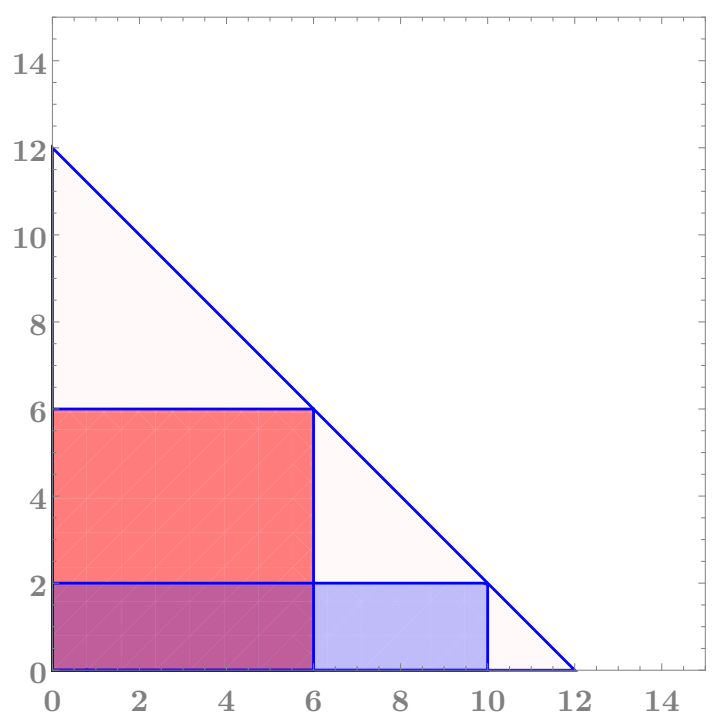

Figure 2. $\left(h^{\prime}, \bar{h}^{\prime}\right)$ plane: asymptotically, the rectangular region (blue shaded) contains exponentially less number of operators compared to square region (red shaded). They are contained within the rightangled triangle, created by $h^{\prime}$ axis, $\bar{h}^{\prime}$ axis and $h^{\prime}+\bar{h}^{\prime}=\Delta$ line. Here $\Delta=12$. 
where for $3 / 8<\alpha \leq 1 / 2$, we have, :

$$
\begin{aligned}
& \left\{\begin{array}{l}
\delta \simeq h^{\alpha} \\
\bar{\delta} \simeq \bar{h}^{\alpha}
\end{array} \quad s(\delta, \bar{\delta}, h, \bar{h})=\log \left(\frac{\sinh \left(\pi \sqrt{\frac{c}{6}} \frac{\delta}{\sqrt{h}}\right)}{\pi \sqrt{\frac{c}{6}} \frac{\delta}{\sqrt{h}}}\right)+\log \left(\frac{\sinh \left(\pi \sqrt{\frac{c}{6}} \frac{\bar{\delta}}{\sqrt{h}}\right)}{\pi \sqrt{\frac{c}{6}} \frac{\bar{\delta}}{\sqrt{h}}}\right)+O\left(\tau^{3 / 4-2 \alpha}\right),\right. \\
& \delta, \bar{\delta} \simeq O(1) \quad s_{-}(\delta, \bar{\delta}) \leq s(\delta, \bar{\delta}, h, \bar{h}) \leq s_{+}(\delta, \bar{\delta})
\end{aligned}
$$

where the functions $s_{ \pm}(\delta, \bar{\delta})$ are determined in the section 3 , in particular, we have $s_{ \pm} \equiv$ $\exp \left(c_{ \pm}\right)$, and $c_{ \pm}$is given by (3.25). We remark that when the bin size is large, there is a universal correction to Cardy formula given by the sinhyperbolic functions. This is analogous to what is found in [2] from the analysis sensitive to dimension only.

Windowed entropy with respect to $\boldsymbol{\Delta}+\boldsymbol{J}$. One can define a microcanonical entropy with respect to $\Delta+J=2 \max \{h, \bar{h}\}$ (name this parameter $\kappa$ ) as

$$
S_{\delta}^{\kappa} \equiv \log [F(\kappa / 2+\delta, \kappa / 2+\delta)-F(\kappa / 2-\delta, \kappa / 2-\delta)],
$$

The asymptotic behavior of $S_{\delta}^{\kappa}$ is given by

$$
S_{\delta}^{\kappa}=4 \pi \sqrt{\frac{c \kappa}{12}}+\log \left(\frac{2 \delta}{\pi \kappa}\right)+s(\delta, \kappa),
$$

where for large enough bin size $\left(\delta \simeq \kappa^{\alpha}\right)$ we have

$$
\delta \simeq \kappa^{\alpha}: s(\delta, \tau)=\log \left(\frac{\sinh \left(2 \pi \sqrt{\frac{c}{3}} \frac{\delta}{\sqrt{\kappa}}\right)}{2 \pi \sqrt{\frac{c}{3}} \frac{\delta}{\sqrt{\kappa}}}\right)+O\left(\kappa^{1 / 4-\alpha}\right), 1 / 4<\alpha \leq 1 / 2 .
$$

\section{$1.2 c>1$ CFTs-results specific for primaries}

One can make the results in the previous subsection specific to Virasoro primaries only, in fact do better. This boils down essentially repeating the argument presented in section 3 , section 4 and section 5 with minor modification. The idea of extending the argument from section 3, section 4 and section 5 to this case is similar in spirit and practice to how [2] obtained the specific results for primary using methods suitable to study all the operators. The details can be found in section 3, specifically eq. (3.37) onwards. Without much ado, here is the result: for finite central charge $c$, we find the integrated density of states specific for primaries behave like (from now on, we will be using the superscript "Vir" to denote the result specific for primaries):

$$
\begin{aligned}
F^{\operatorname{Vir}}(h, \bar{h}) & \equiv \int_{0}^{h} \mathrm{~d} h^{\prime} \int_{0}^{\bar{h}} \mathrm{~d} \bar{h}^{\prime} \rho^{\operatorname{Vir}}\left(h^{\prime}, \bar{h}^{\prime}\right) \\
& =\frac{1}{h, \bar{h} \rightarrow \infty}\left(\frac{3}{\pi^{2}}\right) \exp \left[2 \pi\left(\sqrt{\frac{(c-1) h}{6}}+\sqrt{\frac{(c-1) \bar{h}}{6}}\right)\right]\left[1+O\left(\tau^{-1 / 4}\right)\right]
\end{aligned}
$$


The "windowed" entropy (we have considered bin of size $2 \delta$ by $2 \bar{\delta}$ just like what we did for the analysis of all the operators) for Virasoro primaries is given by

$$
\begin{aligned}
S_{\delta, \bar{\delta}}^{\mathrm{Vir}} & \equiv \log \left(\int_{h-\delta}^{h+\delta} \mathrm{d} h^{\prime} \int_{\bar{h}-\bar{\delta}}^{\bar{h}+\bar{\delta}} \mathrm{d} \bar{h}^{\prime} \rho^{\operatorname{Vir}}\left(h^{\prime}, \bar{h}^{\prime}\right)\right) \\
& =2 \pi\left(\sqrt{\frac{(c-1) h}{6}}+\sqrt{\frac{(c-1) \bar{h}}{6}}\right)-\frac{1}{2} \log \left[\frac{h \bar{h}}{4 \delta^{2} \bar{\delta}^{2}}\right]+s^{\operatorname{Vir}}(\delta, \bar{\delta}, h, \bar{h}),
\end{aligned}
$$

where for $1 / 8<\alpha \leq 1 / 2$, we have:

$$
\begin{aligned}
& \left\{\begin{array}{l}
\delta \simeq h^{\alpha} \\
\bar{\delta} \simeq \bar{h}^{\alpha}
\end{array} \quad: s^{\operatorname{Vir}}(\delta, \bar{\delta}, h, \bar{h})\right. \\
& \quad=\log \left(\frac{\sinh \left(\pi \sqrt{\frac{c-1}{6}} \frac{\delta}{\sqrt{h}}\right)}{\pi \sqrt{\frac{c-1}{6}} \frac{\delta}{\sqrt{h}}}\right)+\log \left(\frac{\sinh \left(\pi \sqrt{\frac{c-1}{6}} \frac{\bar{\delta}}{\sqrt{h}}\right)}{\pi \sqrt{\frac{c-1}{6}} \frac{\bar{\delta}}{\sqrt{h}}}\right)+O\left(\tau^{1 / 4-2 \alpha}\right), \\
& \delta, \bar{\delta} \simeq O(1): s_{-}(\delta, \bar{\delta}) \leq s^{\operatorname{Vir}}(\delta, \bar{\delta}, h, \bar{h}) \leq s_{+}(\delta, \bar{\delta})
\end{aligned}
$$

where the functions $s_{ \pm}(\delta, \bar{\delta})$ are the same functions that appear in the analysis for all the operators.

Large spin, large twist sector for primaries. If we assume a finite twist gap (as defined in 1.1), the result given in eq. (1.12) is true irrespective of whether $h$ and $\bar{h}$ are of the order one or not. Thus unlike the case for all the operators, here we can trust the polynomially suppressed correction for all values of $v$, where $h=\bar{h}^{v}$.

\subsection{Large spin, finite twist sector}

The large spin, finite twist sector is not entirely asymptotic regime since the quantity knows about low lying spectrum in one of the weights. It turns out we can only put an upper bound in this case. There is an $O(1)$ error in the estimation. While for the upper bound this does not cause any trouble, for the lower bound, it makes thing tricky. In particular, the lower bound on the density of states, appropriately integrated, contains an exponential piece as expected from extended Cardy formula $[4,5,7,8]$ but it comes with a multiplicative order one number, which can become negative unless proven otherwise.

Analysis for all the operators. In what follows, we will keep $h$ finite and let $\bar{h} \rightarrow \infty$, the windowed entropy $S_{\delta, \bar{\delta}}^{\mathrm{ft}}$ is found to be bounded above by

$$
S_{\delta, \bar{\delta}}^{\mathrm{ft}} \leq \mathbb{S}_{h, \delta, \bar{\delta}}^{\mathrm{ft}} \leq 2 \pi \sqrt{\frac{c \bar{h}}{6}}-\frac{1}{4} \log \left(\frac{\bar{h}^{3}}{16 \bar{\delta}^{4}}\right)+M
$$


where $M$ is an order one number. Here $S_{\delta, \bar{\delta}}^{\mathrm{ft}}$ and $\mathbb{S}_{h, \delta, \bar{\delta}}^{\mathrm{ft}}$ are defined as

$$
\exp \left[S_{\delta, \bar{\delta}}^{\mathrm{ft}}\right] \equiv \int_{h-\delta}^{h+\delta} \mathrm{d} h^{\prime} \int_{\bar{h}-\bar{\delta}}^{\bar{h}+\bar{\delta}} \mathrm{d} \bar{h}^{\prime} \rho\left(h^{\prime}, \bar{h}^{\prime}\right), \quad \exp \left[\mathbb{S}_{h, \delta, \bar{\delta}}^{\mathrm{ft}}\right] \equiv \int_{0}^{h+\delta} \mathrm{d} h^{\prime} \int_{\bar{h}-\bar{\delta}}^{\bar{h}+\bar{\delta}} \mathrm{d} \bar{h}^{\prime} \rho\left(h^{\prime}, \bar{h}^{\prime}\right) .
$$

The number $M$ is given (or estimated) by

$$
M=2 \pi\left(h+\delta-\frac{c}{24}\right)+\log \left[c_{+} \sum_{\tilde{h}} \chi_{\tilde{h}}\left(e^{-2 \pi}\right)\right]
$$

where $\chi_{\tilde{h}}$ is the character for the conserved current with weight $(\tilde{h}, 0), \tilde{h} \geq 0$ (including the Identity) and $c_{ \pm}$is an order one $h, \bar{h}$ independent number, defined in section 7. $M$ is a finite number as the absolute value of the sum over $\tilde{h}$ is bounded above by the partition function evaluated at $\beta=\bar{\beta}=2 \pi$, which is a finite number.

Analysis for primaries with/without conserved currents. The above result can also be made specific to primaries:

$$
S_{\delta, \bar{\delta}}^{\mathrm{Vir}, \mathrm{ft}} \leq \mathbb{S}_{h, \delta, \bar{\delta}}^{\mathrm{Vir}, \mathrm{ft}} \leq 2 \pi \sqrt{\frac{(c-1) \bar{h}}{6}}-\frac{1}{2} \log \left(\frac{\bar{h}}{4 \bar{\delta}^{2}}\right)+M^{\mathrm{Vir}}
$$

Here $S_{\delta, \bar{\delta}}^{\mathrm{Vir}, \mathrm{ft}}$ and $\mathbb{S}_{h, \delta, \bar{\delta}}^{\mathrm{Vir}, \mathrm{ft}}$ are defined as

$$
\begin{aligned}
\exp \left[\mathbb{S}_{h, \delta, \bar{\delta}}^{\mathrm{Vir}, \mathrm{ft}}\right] & \equiv \int_{0}^{h+\delta} \mathrm{d} h^{\prime} \int_{\bar{h}-\bar{\delta}}^{\bar{h}+\bar{\delta}} \mathrm{d} \bar{h}^{\prime} \rho^{\mathrm{Vir}}\left(h^{\prime}, \bar{h}^{\prime}\right), \\
\exp \left[S_{\delta, \bar{\delta}}^{\mathrm{Vir}, \mathrm{ft}}\right] & \equiv \int_{h-\delta}^{h+\delta} \mathrm{d} h^{\prime} \int_{\bar{h}-\bar{\delta}}^{\bar{h}+\bar{\delta}} \mathrm{d} \bar{h}^{\prime} \rho^{\mathrm{Vir}}\left(h^{\prime}, \bar{h}^{\prime}\right) .
\end{aligned}
$$

and $M^{\mathrm{Vir}}$ is an order one number, given by

$$
M^{\mathrm{Vir}}=2 \pi\left(h+\delta-\frac{c-1}{24}\right)+\log \left[c_{+} \sum_{\tilde{h}} e^{-2 \pi\left(\tilde{h}-\frac{c-1}{24}\right)}\right]
$$

where the zero twist primaries have weight $(\tilde{h}, 0), \tilde{h} \geq 0$ (including the Identity) and $c_{+}$is an order one $h, \bar{h}$ independent number, defined in section 7. $M^{\mathrm{Vir}}$ is a finite number since the sum inside the log is convergent. This happens because the absolute value of the sum is bounded by the partition function evaluated at $\beta=\bar{\beta}=2 \pi$, which is a finite number.

Analysis for primaries for CFT with no nontrivial conserved current. If we assume that there is no nontrivial conserved current i.e. the only zero twist primary is the Identity and there is a finite twist gap (the finite twist gap as defined in 1.1, combined with the absence of nontrivial conserved current implies the usual twist gap condition used in 
the literature, for example in [20]). we show that

$$
\begin{aligned}
S_{\delta, \bar{\delta}}^{\mathrm{Vir}, \mathrm{ft}} \leq \mathbb{S}_{h, \delta, \bar{\delta}}^{\mathrm{Vir}, \mathrm{ft}} \leq & 2 \pi \sqrt{\frac{(c-1) \bar{h}}{6}}-\frac{1}{2} \log \left(\frac{\bar{h}}{4 \bar{\delta}^{2}}\right)+\log \left(\sqrt{h+\delta-\frac{c-1}{24}}\right) \\
& +\frac{\pi^{2}}{6}(c-1)\left(h+\delta+\frac{c-1}{24}\right)+\log \left(1-e^{-4 \pi^{2}\left(h+\delta-\frac{c-1}{24}\right)}\right)+M^{\prime}
\end{aligned}
$$

where $M^{\prime}$ is an order one $h$ independent number. If we assume that $\left(h+\delta-\frac{c-1}{24}\right)$ is a very small number compared to $\frac{1}{c-1}$, this matches with the leading result appeared in the lightcone bootstrap program [4-8] i.e.

$$
S_{\delta, \bar{\delta}}^{\mathrm{Vir}, \mathrm{ft}} \leq \mathbb{S}_{h, \delta, \bar{\delta}}^{\mathrm{Vir}, \mathrm{ft}} \leq 2 \pi \sqrt{\frac{(c-1) \bar{h}}{6}}-\frac{1}{2} \log \left(\frac{\bar{h}}{4 \bar{\delta}^{2}}\right)+\frac{3}{2} \log \left(h+\delta-\frac{c-1}{24}\right)+\tilde{M}^{\prime}
$$

where $\tilde{M}^{\prime}=M+\log \left(4 \pi^{2}\right)$. We remark that the limit is very subtle here. There are several scales. The scale set by $\bar{h}$ is the largest one and we are seeking an asymptotic behavior in $\bar{h}$. Then there are two fixed parameters $h$ and $c$. We are probing the regime where $\left(h+\delta-\frac{c-1}{24}\right)$ is a very small number compared to $\frac{1}{c-1}$. The details of the calculation can be found at the end of section 7 .

\subsection{Asymptotic spectral gap}

The idea about deriving an upper bound on spectral gap comes from binning the states. If we make the bin size very small, we can not prove a positive lower bound on the number of states in that bin, because the bin might not have any state at all. As we increase the bin size, the chances are more that we find such positive lower bound. If we find a positive lower bound for a specific bin size centered at some large $h, \bar{h}$; that would immediately imply existence of an upper bound on the asymptotic spectral gap.

\subsubsection{Probing spectral gap via circle of order one area}

With/without twist gap. Here we do not put any assumption on twist gap.

Let us consider a square $\mathbb{S}$ of side $\frac{4 \sqrt{3} \gamma}{\pi}+\epsilon_{g}$ centered at $(h, \bar{h})$ on $\left(h^{\prime}, \bar{h}^{\prime}\right)$ plane. Here $\epsilon_{g}$ can be any arbitrarily small positive number. In the limit $h \rightarrow \infty, \bar{h} \rightarrow \infty$ we have

$$
\int_{\mathbb{S}} \mathrm{d} h^{\prime} \mathrm{d} \bar{h}^{\prime} \rho^{\operatorname{Vir}}\left(h^{\prime}, \bar{h}^{\prime}\right)>0
$$

where the asymptotic region is reached along a curve for which $\max (h, \bar{h}) \simeq \frac{\gamma^{4} \tau}{2}$. Thus the spectral gap along this curve is bounded above by a circle of radius $\frac{\gamma r}{\sqrt{2}}$ and the best possible value of $r$ that we find is $r=\frac{4 \sqrt{3}}{\pi}$, this being the circle circumscribing the square. 
An immediate corollary is that the asymptotic twist gap is upper bounded by $\frac{8 \sqrt{3} \gamma}{\pi} \simeq$ $4.42 \gamma$. For $c>1$, the argument can be made specific for primaries, hence the asymptotic gap. This in some sense complements the bound on primary twist gap over the vacuum $^{4}[8,20]$.

We suspect that either by suitable choice of function or by the better estimate of heavy sector of the partition function, $r$ and/or length of a side of the bounding square can be made to 1 . If this can be done, then the bound becomes optimal for $\gamma=1$, (assuming we always consider circular/square region) since tensoring chiral Monster CFT with antichiral Monster CFT saturates the bound. One can see the saturation by circumscribing a square of unit length by a circle of radius $\frac{1}{\sqrt{2}}$ on $\left(h^{\prime}, \bar{h}^{\prime}\right)$ plane [see the figure 3]. Nonetheless, the optimality along a curve for $\gamma \neq 1$ is not guaranteed. An immediate corollary of finding such a circle is that the asymptotic twist gap is upper bounded by $2 \sqrt{2}$ along $h=\bar{h}$ curve. The same bound holds for asymptotic primary twist gap. We remark that in terms of twist, the above gap might not be optimal, since if we tensor chiral Monster CFT with anti-chiral monster CFT, the asymptotic twist gap is 2 . If one can find a bounding square of side length given by 1 , that would reproduce the optimal twist gap 2 .

The above result and the conjectures can not be applied to a scenario, where infinity is approached along a curve where $h$ is of widely different order compared to $\bar{h}$, in particular, say, if we want to approach the infinity along the curve $h^{v}=\bar{h}$ with $v \neq 1$. To circumnavigate this issue, we assume existence of twist gap $g$. We remind the readers that by finite twist gap, we mean there exists a number $\tau_{*}>0$ such that there is no operator with twist $\tau \in\left(0, \tau_{*}\right)$ and there are finite number of zero twist operators with dimension less than $c / 12$, and $g \geq \tau_{*}$. Moreover, assuming existence of $g$ helps us to get rid of dependence on $\gamma$.

CFT with twist gap $\boldsymbol{g}$. Now we assume that the CFT has a twist gap as defined in 1.1.

For a CFT with twist gap $g$ (as defined in 1.1) and central charge $c>1$, one can have a bounding circle $\mathbb{C}$ specific to primaries having a radius $\frac{\sigma r}{\sqrt{2}}+\epsilon_{g}$ with $\epsilon_{g}>0$, where

$$
\sigma=\max \left(1, \sqrt{\frac{c-1}{12 g}}\right), \quad r=\frac{4 \sqrt{3}}{\pi} .
$$

irrespective of how infinity is approached, such that the bounding circle contains at least one operator.

Thus for such a scenario there exists $h_{*}$ and $\bar{h}_{*}$, two order one numbers such that

$$
\int_{\mathbb{C}} \mathrm{d} h^{\prime} \mathrm{d} \bar{h}^{\prime} \rho^{\operatorname{Vir}}\left(h^{\prime}, \bar{h}^{\prime}\right)>0, \forall h>h_{*}, \bar{h}>\bar{h}_{*} .
$$

Again this is obtained by circumscribing the appropriate bounding square [see the figure 4]. The superscript "Vir" on $\rho^{\mathrm{Vir}}$ denotes that it is density of primary operators as opposed to

\footnotetext{
${ }^{4}$ In [20], it is mentioned that the argument is due to Tom Hartman.
} 


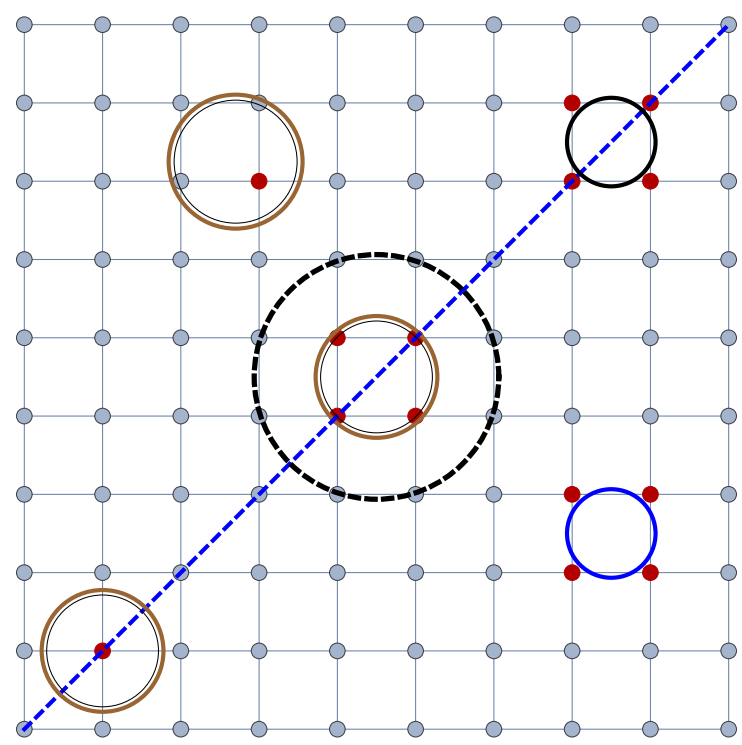

Figure 3. Operator spectrum of chiral Monster CFT tensored with its antichiral avatar on $\left(h^{\prime}, \bar{h}^{\prime}\right)$ plane: each vertex in the lattice represents the presence of operators. One can always circumscribe a square of unit length by a circle of radius $\frac{1}{\sqrt{2}}$. Thus any circle centered at $(h, \bar{h})$ and of radius $\frac{1}{\sqrt{2}}+\epsilon_{g}$ with $\epsilon_{g}>0$ would contain at least an operator. This is consistent with our result that asymptotically any circle of radius $\frac{\gamma r}{\sqrt{2}}+\epsilon_{g}$ with $\gamma \geq 1$ will contain at least one operator where $\gamma$ is defined as the fourth root of asymptotic ratio of $\max (h, \bar{h})$ and $\min (h, \bar{h})$. We show $r=\frac{4 \sqrt{3}}{\pi}>1$ and suspect that $r$ can be made to 1 . Here we have shown three such examples of containment. For each choice, two circles with different radius have been drawn to show that a circle of $\frac{\gamma}{\sqrt{2}}+\epsilon_{g}$ will contain at least one operator, as long as $\epsilon_{g}>0$. On the diagonal we have $\gamma=1$. The red dots denote the contained operators. The black circle shows that one can not ensure containment with circle of radius less than $\frac{1}{\sqrt{2}}$, showing optimality along the $h=\bar{h}$ curve if one can show $r=1$. We remark that as we have only shown that $r=\frac{4 \sqrt{3}}{\pi}>1$ (the dashed black circle), we have not yet reached the optimal bound. The blue circle suggests that the optimal bound should be $\frac{1}{\sqrt{2}}$ even for the case where $h \neq \bar{h}$. Assuming a twist gap makes this bound insensitive to the line of approach towards infinity, but sensitive to the gap. See figure 4 .

all the operators. In a compact unitary $2 \mathrm{D}$ CFT without any conserved current, one can use the upper bound of twist gap due to Hartman, appearing in [20], to deduce

$$
\sigma=\max \left(1, \sqrt{\frac{c-1}{12 g}}\right)=\sqrt{\frac{c-1}{12 g}} .
$$




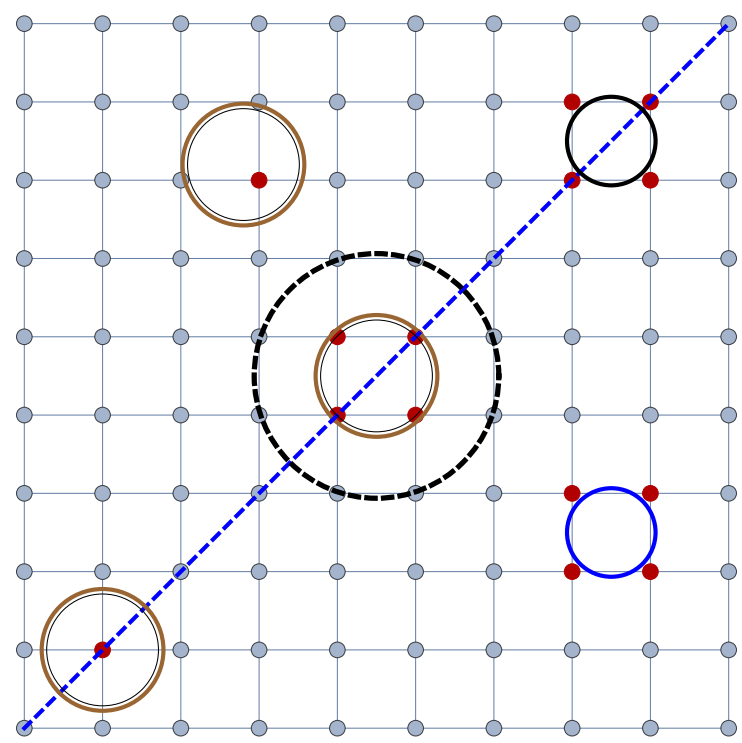

Figure 4. Assuming twist gap g: operator spectrum of chiral Monster CFT tensored with its antichiral avatar on $\left(h^{\prime}, \bar{h}^{\prime}\right)$ plane: each vertex in the lattice represents the presence of operators. One can always circumscribe a square of unit length by a circle of radius $\frac{1}{\sqrt{2}}$. Thus any circle centered at $(h, \bar{h})$ and of radius $\frac{\kappa}{\sqrt{2}}+\epsilon_{g}$ with $\epsilon_{g}>0, \kappa \geq 1$ would contain at least an operator. This is consistent with our result that asymptotically any square of side length $\frac{4 \sqrt{3}}{\pi}$, hence any circle of radius $\frac{\sigma r}{\sqrt{2}}+\epsilon_{g}$ will contain at least one operator, where $\sigma=\max \left(\sqrt{\frac{c-1}{12 g}}, 1\right)$ and $r=\frac{4 \sqrt{3}}{\pi}>1$. Again we suspect that $r$ can be made to 1 . In the Monster example $g=4$, hence $\sigma=1$.

Now we will explain the sense in which the minimal gap is complementary to twist gap:

Suppose we consider a 2D compact unitary CFT with twist gap $g$ such that it does not have any zero twist primaries (conserved currents) except the Identity: if asymptotically there exists a circle of minimal area ${ }^{a} A$ on $\left(h^{\prime}, \bar{h}^{\prime}\right)$ plane such that it does not contain any operator, we immediately deduce the following inequality

$$
A g \leq \frac{\pi(c-1) r^{2}}{24}
$$

${ }^{a}$ One can imagine that operators having conformal weight $(h, \bar{h})$ are denoted by the point $(h, \bar{h})$ on $\left(h^{\prime}, \bar{h}^{\prime}\right)$ plane. We name this set to be $\mathbb{S}$. Now consider the set $\mathbb{S}_{d} \equiv\{d(a, b): a, b \in \mathbb{S}\}$, where $d(a, b)$ is the Euclidean distance on the plane between the points $a$ and $b$. Existence of a circle with minimal area means Inf $\mathbb{S}_{d}>0$. Asymptotically minimal area means that we look at the plane for $h^{\prime}>h_{*} \& \bar{h}^{\prime}>\bar{h}_{*}$ and construct the set $\mathbb{S}_{d}$ and consider its infimum.

This can be thought of as an upper bound on twist gap if the minimal areal gap $A$ is known (note that minimal areal gap obtained from the full spectra has to be less than or equal to the asymptotic minimal gap). If one can make $r=1$ and show that $\frac{\pi}{2}<A=\frac{k \pi}{2}$ then it is possible to lower the upper bound on twist gap from $\frac{c-1}{12}$ to $\frac{c-1}{12 k}$ with $k>1$. This might be of importance for proving the proposed upper bound $\frac{c-1}{16}$ in [8], if it is true. To rephrase, if one can show that the minimal area $A=\frac{2 \pi}{3}$ (thus the diameter of the circle 


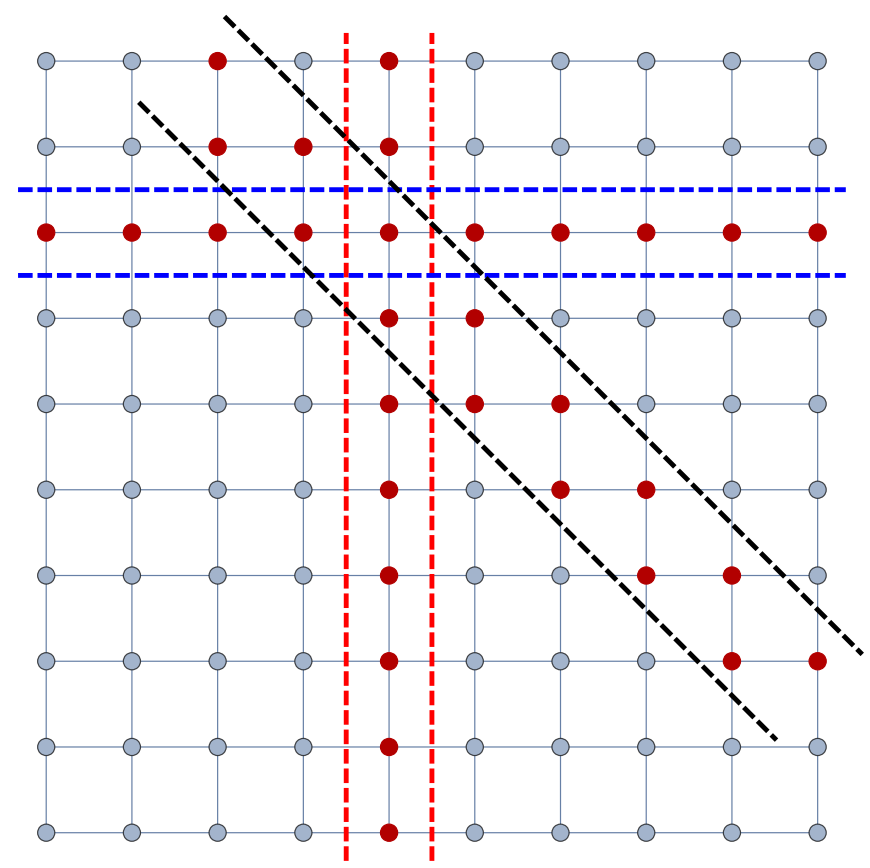

Figure 5. We consider three kind of strips: the red vertical one is $H_{2}(\bar{h})$, the blue horizontal one is $H_{1}(h)$ and the black one is $H_{3}(\Delta)$. In each cases, we see that there is a minimum width of the strip such that the strips contain at least one operator. If we tensor chiral Monster CFT with its anti-chiral avatar, we can not make the width of the horizontal and vertical strip less than 1 . Thus the bound we find (which is $\sqrt{2}$ ) might not be optimal. A technical remark is that if it is not optimal, that would hint that one could have estimated the heavy part of the partition function in a better way.

would be $2 \sqrt{\frac{2}{3}}$ ), it would imply the proposed bound. Similarly, any lower bound on twist gap translates to upper bound on minimal areal gap $A$.

The similar analysis can be done for all the operators assuming a twist gap. The only difference is that $\sigma$ would be given by $\sigma=\max \left(1, \sqrt{\frac{c}{12 g}}\right)$. We elucidate on these bounds in the section 3 .

\subsubsection{Probing spectral gap via strips}

Instead of squares, we can think of covering the $\left(h^{\prime}, \bar{h}^{\prime}\right)$ plane via strips of finite width and ask what is the minimum width of the strip that guarantees existence of at least one state in the strip. We can consider three kind of strips (see figure 5):

$$
\begin{aligned}
H_{1}(h) & \equiv\left\{\left(h^{\prime}, \bar{h}^{\prime}\right): h^{\prime} \in\left[h-\delta_{1}, h+\delta_{1}\right], \bar{h}^{\prime} \geq 0\right\}, \\
H_{2}(\bar{h}) & \equiv\left\{\left(h^{\prime}, \bar{h}^{\prime}\right): \bar{h}^{\prime} \in\left[\bar{h}-\delta_{2}, \bar{h}+\delta_{2}\right], h^{\prime} \geq 0\right\}, \\
H_{3}(\Delta) & \equiv\left\{\left(h^{\prime}, \bar{h}^{\prime}\right): h^{\prime}+\bar{h}^{\prime} \in\left[\Delta-\delta_{3}, \Delta+\delta_{3}\right], h^{\prime}, \bar{h}^{\prime} \geq 0\right\} .
\end{aligned}
$$

It is shown in [3] that if $\delta_{3}>\frac{1}{2}$, we have

$$
\int_{H_{3}(\Delta \rightarrow \infty)} \mathrm{d} h^{\prime} \mathrm{d} \bar{h}^{\prime} \rho\left(h^{\prime}, \bar{h}^{\prime}\right)>0 .
$$

Thus the asymptotic spectral gap is bounded above by 1 . 
In this work we show that

$$
\int_{H_{1}(h \rightarrow \infty)} \mathrm{d} h^{\prime} \mathrm{d} \bar{h}^{\prime} \rho^{\operatorname{Vir}}\left(h^{\prime}, \bar{h}^{\prime}\right)>0 \text { for } \delta_{1}>\frac{1}{\sqrt{2}} .
$$

The same result holds true for the $H_{2}$ strip with $h$ replaced by $\bar{h}$, where $H_{1}, H_{2}$ are defined in (1.26). This comes from putting a positive lower bound on the right hand side of the following inequality:

$$
\int_{H_{1}(h \rightarrow \infty)} \mathrm{d} h^{\prime} \mathrm{d} \bar{h}^{\prime} \rho\left(h^{\prime}, \bar{h}^{\prime}\right) \geq \int_{H_{1}(h \rightarrow \infty)} \mathrm{d} h^{\prime} \mathrm{d} \bar{h}^{\prime} \rho\left(h^{\prime}, \bar{h}^{\prime}\right) e^{-\bar{\beta} \bar{h}} .
$$

We achieve this as a corollary of the lemma proven in section 4. This shows that

Asymptotically on the $\left(h^{\prime}, \bar{h}^{\prime}\right)$ plane, if the width of the horizontal or vertical strip is bigger than $\sqrt{2}$, it contains at least one Virasoro primary. This might not be optimal since the gap we find by tensoring chiral Monster CFT with its anti-chiral avatar is 1 .

\subsection{Analysis at large central charge}

We consider the $c \rightarrow \infty$ limit and parametrize the conformal weights in following way:

$$
h=c\left(\frac{1}{24}+\epsilon\right), \bar{h}=c\left(\frac{1}{24}+\bar{\epsilon}\right) ; \quad \epsilon, \bar{\epsilon} \text { fixed }
$$

Let us define $\epsilon_{*}=\min (\epsilon, \bar{\epsilon})$ and $\epsilon^{*}=\max (\epsilon, \bar{\epsilon})$.

With/without twist gap. We show that ${ }^{5}$

For $\min \{(h / c-1 / 24),(\bar{h} / c-1 / 24)\}=\epsilon_{*}>\frac{1}{6}$, the microcanonical entropy for order one window $\delta, \bar{\delta} \simeq O(1)$ is given by

$$
S_{\delta, \bar{\delta}} \underset{c \rightarrow \infty}{\simeq} 2 \pi\left(\sqrt{\frac{c}{6}\left(h-\frac{c}{24}\right)}+\sqrt{\frac{c}{6}\left(\bar{h}-\frac{c}{24}\right)}\right)-\log (c)+O(1),
$$

while for $\delta, \bar{\delta} \simeq c^{\alpha}$ with $0<\alpha \leq 1$, we have

$$
S_{\delta} \underset{c \rightarrow \infty}{\simeq} 2 \pi\left(\sqrt{\frac{c}{6}\left(h+\delta-\frac{c}{24}\right)}+\sqrt{\frac{c}{6}\left(\bar{h}+\bar{\delta}-\frac{c}{24}\right)}\right)-\log (c)+O(1)
$$

\footnotetext{
${ }^{5}$ It might be possible to extend the region of validity beyond this, in particular, following [19], one might expect it to be valid for $\epsilon \bar{\epsilon}>\frac{1}{24^{2}}$ !
} 
Assuming finite twist gap. If we assume a finite twist gap $g$ (as defined in 1.1), then

The regime of validity of the Cardy result as in (1.33) can be extended to

$$
\epsilon_{*}>\frac{1}{6}\left[\max \left\{\frac{1}{2},\left(1-\frac{6 g}{c}\right)^{2}\right\}\right], \quad \epsilon_{*} \equiv \min \{(h / c-1 / 24),(\bar{h} / c-1 / 24)\} .
$$

Relevant recent work. There has been recent surge in analyzing the asymptotics of CFT data on a rigorous footing. The results have been obtained [22, 23] using techniques borrowed from a part of mathematics literature, which goes by the name of Tauberian theorems. The appendix C of [24] emphasizes the importance of Ingham theorem [25] in analyzing Cardy's result [1] for the asymptotic density of states in 2D CFT. Subsequently, the complex Tauberian theorems, as appeared originally in [26] is utilized in the work of [27]. A complete rigorous treatment of Cardy formula appeared in the work [2], where they figured out the density of states in $\Delta \rightarrow \infty$ limit with a rigorous optimal estimate of the error term. The improvement of the result along with a proof of the conjecture made in [2] has been put forward in [3]. An rigorous analysis of the asymptotics of three point coefficients [28] appeared recently [29] where the main challenge was to circumnavigate the negativity issue for the analysis of three point coefficients.

\section{Set up}

We consider a 2D CFT with spectrum of operators having conformal weights $\left(h^{\prime}, \bar{h}^{\prime}\right)$. We assign different real temperatures $\beta, \bar{\beta}$ to the left-moving and the right-moving sectors respectively. The partition function $Z(\beta, \bar{\beta})$ is given by:

$$
Z(\beta, \bar{\beta})=\sum_{h^{\prime}, \bar{h}^{\prime}} e^{-\beta\left(h^{\prime}-c / 24\right)-\bar{\beta}\left(\bar{h}^{\prime}-c / 24\right)} .
$$

The modular invariance of the partition function yields:

$$
Z(\beta, \bar{\beta})=Z\left(\beta^{\prime}, \bar{\beta}^{\prime}\right), \quad \beta^{\prime}=\frac{4 \pi^{2}}{\beta}, \quad \bar{\beta}^{\prime}=\frac{4 \pi^{2}}{\bar{\beta}} .
$$

We further define the following measure

$$
\mathrm{d} F\left(h^{\prime}, \bar{h}^{\prime}\right)=\sum d\left(h_{i}, \bar{h}_{i}\right) \delta\left(h^{\prime}-h_{i}\right) \delta\left(\bar{h}^{\prime}-\bar{h}_{i}\right)
$$

where $d\left(h_{i}, \bar{h}_{i}\right)$ is the degeneracy of the state with conformal weight $\left(h_{i}, \bar{h}_{i}\right)$. Our goal is to estimate the integral of the measure $\mathrm{d} F$ over different regions.

\subsection{A semi technical glimpse of the subtleties}

One of the key step in deriving the Cardy formula is the intuitive understanding that at high temperature, the partition function is dominated by the heavy states, thus doing an 
inverse Laplace transform of the high temperature behavior of the partition function should produce the asymptotic density of states. Schematically,

$$
Z(\beta \rightarrow 0)=\underbrace{\text { Leading Term }}_{\text {Produces asymptotic density of states }}+\text { Error },
$$

Inverse Laplace [Error] $\stackrel{? ? ?}{=}$ Error in asymptotic density of states .

The underlying assumption while doing the above is that the inverse Laplace transform of the error term is bounded as well, thus producing an error compared to the leading behavior of the asymptotic density of states. The Tauberian formalism justifies this step by carefully estimating the error terms. The way it works is following: one bounds the number of states within an order an window centered at some heavy $\Delta$, from above and below by some convolution $(\circledast)$ of partition function at high temperature $(\beta)$ and bandlimited function $\phi_{ \pm}$, schematically this looks like

$$
\left[Z(\beta+i t) \circledast \phi_{-}(t)\right] \leq \# \text { states in window } \leq\left[Z(\beta+i t) \circledast \phi_{+}(t)\right] .
$$

Intuitively, at this stage, we know that heavy states contribute to this partition function at high temperature. Now we implement modular transformation, the partition function at high temperature becomes partition function at low temperature. Schematically we have

$$
\left[Z\left(\frac{4 \pi^{2}}{\beta+i t}\right) \circledast \phi_{-}(t)\right] \leq \# \text { states in window } \leq\left[Z\left(\frac{4 \pi^{2}}{\beta+i t}\right) \circledast \phi_{+}(t)\right] .
$$

At low temperature, low lying states contribute the most i.e. $Z\left(\frac{4 \pi^{2}}{\beta} \rightarrow \infty\right)$ is dominated by low lying states. So, following [19], we separate the low lying states from heavy states; the low lying states constitute the "light" part, while the "heavy" part is complement of that. The "light" part contains finite number of operators at finite central charge. So we do the inverse Laplace transform of this "Light" part to get the leading answer $\rho_{*}$. Thus $\rho_{*}$ reproduces the $Z\left(\frac{4 \pi^{2}}{\beta} \rightarrow \infty\right)$ behavior upon doing Laplace transformation. We are still left with the "heavy" part contribution of $Z\left(\frac{4 \pi^{2}}{\beta+i t}\right)$. This part can be shown to produce a subleading correction to the leading piece of asymptotic density of states, thus justifying (2.5) using the bound proven in [19]. This requires relating the "heavy" part at temperature $\beta$ to the "light" part at temperature $\beta^{\prime}$. The upshot of this discussion is that we have a full control of the error term and its inverse Laplace transformation. In practice, we only consider the Identity operator among all the operators in the "light" region. So one might worry about the error coming from that but since there are finite number of operators in the "Light" region, one can do inverse Laplace transformation term by term and show that each of them is exponentially suppressed and hence the finite sum of them. We emphasize the "finiteness" of finite sum is really very important for this and this is precisely why we need to treat heavy part separately. ${ }^{6}$ In fact, we remind the readers that

\footnotetext{
${ }^{6}$ One could have imagined doing inverse Laplace transformation term by term including the operators from "heavy" sector, even if each of them is suppressed, there's no guarantee that the infinite sum is suppressed.
} 
in the large central charge limit, we have infinite number of operators even in the "light" sector, hence we need an extra assumption of sparseness, as done in [19].

The immediate generalization of this technique used in the [2] has obstacles because of the cross terms present in the analysis, for example, say contribution from the states where $h^{\prime}$ is large but $\bar{h}^{\prime}$ is not that large. The most obvious way to generalize the argument is to use the generalized HKS [19] cut: dividing the $\left(h^{\prime}, \bar{h}^{\prime}\right)$ plane into two regions, where the "light" region (call it $\mathbb{L}$ ) contains all the operators with one of $h^{\prime}$ or $\bar{h}^{\prime}$ being less than $c / 24$ while the heavy region is the complement of them. It is possible to make a similar statement about this "heavy" region, relating it to the "light" part using HKS like argument. Nonetheless, one then stumbles upon the issue of defining $\rho_{*}$, which is supposed to reproduce the leading contribution to the partition function at high temperature $\left(\beta \rightarrow 0, \beta^{\prime} \rightarrow \infty\right)$, to be precise, the light part of the partition function at temperature $\beta^{\prime}$. Now the issue is that there are infinite number of operators in this region $\mathbb{L}$. Unlike the case in [2], we just can not take the Identity operator to prove that this produces the leading behavior and say the rest are suppressed. In particular, the previous argument of term by term exponential suppression fails because there are infinite of them. The take home message is that it is not a priori clear whether just considering the vacuum to calculate $\rho_{*}$ is good enough, because infinite number of other operators might conspire to spoil the "leading contribution", even if each one of them is exponentially suppressed. We reemphasize that [2] did not face this problem, since in their case, the light region was $\Delta^{\prime}<c / 12$ and the region has finite number of operators and everything is under control, so in principle their $\rho_{*}$ was defined having contribution from all those states with $\Delta^{\prime}<c / 12$ and in practice derivable from the vacuum. To circumnavigate this problem, in section 3 , we use the original HKS cut i.e. we define the "light" region to be the one where $h^{\prime}+\bar{h}^{\prime}<c / 12$ and the "heavy" region is the complement of the light region. See figure 6 . The immediate cost for doing this is that we can make comment only when $h$ and $\bar{h}$ is of the same order asymptotically. The details depend on how infinity is approached. But this is expected because intuitively the infinity can be reached several ways on a plane. This issue persists for the analysis sensitive to primary as well. It turns out that one can bypass this restriction of $h$ and $\bar{h}$ being of the same order, if one assumes a twist gap (as defined in 1.1 we require finite number of conserved currents with dimension less than $c / 12$ to be precise). In that scenario, the results in section 3 holds true even if $h$ and $\bar{h}$ are not of the same order asymptotically. The estimation of the "heavy" region becomes really involved in this case and carried out in details in section 3. One has to further separate out the "heavy" zero twist operators and estimate their contribution separately (see the right figure 6).

We further point out that estimating the integrated density of states require us to prove another lemma, which is special to $2 \mathrm{D}$ Tauberian theorem. We achieve this in section 4. The lemma is then fed into the main proof in section 5. The result for the integrated density of states also requires an estimate of number of states within an order one area on $\left(h^{\prime}, \bar{h}^{\prime}\right)$ plane, which is achieved in section 3 . In section 6 , we verify our results using $2 \mathrm{D}$ Ising model. The large spin, finite twist is discussed in section 7 . The large central charge regime is discussed in section 8 . We conclude with a list of open of problems along with a brief discussion. 

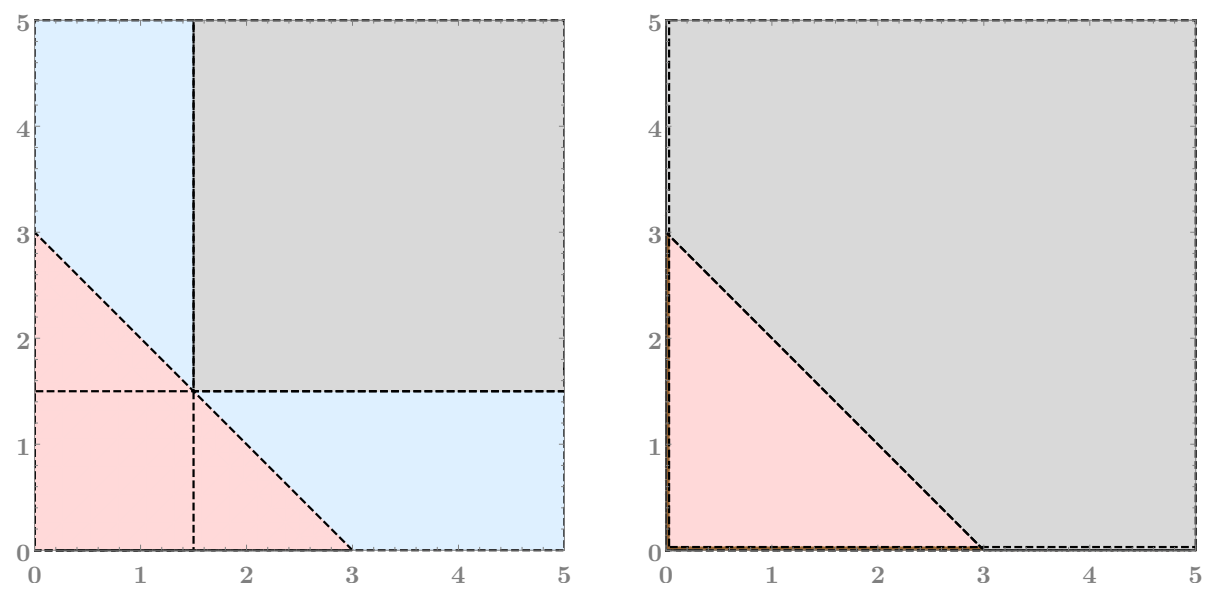

Figure 6. The $\left(h^{\prime}, \bar{h}^{\prime}\right)$ plane: the light red shaded region contributes to $Z_{L}$ and given by the states with $h^{\prime}+\bar{h}^{\prime}<c / 12$. The complement of this contributes to $Z_{H}$. The naive HKS cut makes the union of red and blue shaded region to be the "Light" region. But this brings in the problem of having infinite number of operators in the light region. This cut is applicable to the analysis sensitive to primaries as well with a shifted central charge $c \mapsto c-1$. The picture on the right emphasizes that one needs to treat the zero twist operators separately for some part of the analysis.

\section{$3 \quad O(1)$ rectangular window}

In this section, we study the number of states lying on an order one rectangular/square area of $\left(h^{\prime}, \bar{h}^{\prime}\right)$ plane, centered at some large $(h, \bar{h})$ and having sides of length $2 \delta$ and $2 \bar{\delta}$. We are interested in $h \rightarrow \infty, \bar{h} \rightarrow \infty$ limit with $\delta, \bar{\delta}$ being fixed order one numbers. We do it in two ways, in the first subsection, we do it generically without any assumption on twist gap while in the next subsection, we assume existence of a twist gap. The assumption of the twist gap (as defined in 1.1) facilitates studying the regime where $h$ is not of the same order as $\bar{h}$.

\subsection{Generic analysis: with/without twist gap}

Following [2], let us choose functions $\Phi_{ \pm}(h, \bar{h})$ such that

$$
\Phi_{-}\left(h^{\prime}, \bar{h}^{\prime}\right) \leq \Theta_{h, \bar{h}, \delta, \bar{\delta}}\left(h^{\prime}, \bar{h}^{\prime}\right) \leq \Phi_{+}\left(h^{\prime}, \bar{h}^{\prime}\right),
$$

where $\Theta_{h, \bar{h}, \delta, \delta^{\prime}}\left(h^{\prime}, \bar{h}^{\prime}\right)$ is the indicator function of the rectangle and defined as

$$
\Theta_{h, \bar{h}, \delta, \delta^{\prime}}\left(h^{\prime}, \bar{h}^{\prime}\right)=\theta_{[h-\delta, h+\delta]}\left(h^{\prime}\right) \theta_{[\bar{h}-\bar{\delta}, \bar{h}+\bar{\delta}]}\left(\bar{h}^{\prime}\right),
$$

In principle, one can choose the energy window for the microcanonical ensemble to be of a different shape, for example, a circle. But for now, we consider it to be a rectangle. Now, from eq. (3.1) we obtain

$$
e^{\beta(h-\delta)+\bar{\beta}(\bar{h}-\bar{\delta})} e^{-\beta h^{\prime}-\bar{\beta} \bar{h}^{\prime}} \Phi_{-}\left(h^{\prime}, \bar{h}^{\prime}\right) \leq \Theta_{h, \bar{h}, \delta, \bar{\delta}}\left(h^{\prime}, \bar{h}^{\prime}\right) \leq e^{\beta(h+\delta)+\bar{\beta}(\bar{h}+\bar{\delta})} e^{-\beta h^{\prime}-\bar{\beta} \bar{h}^{\prime}} \Phi_{+}\left(h^{\prime}, \bar{h}^{\prime}\right) .
$$


Multiplying the above by the density of states $\rho\left(h^{\prime}, \bar{h}^{\prime}\right)$ and integrating yields the following inequality:

$$
\begin{aligned}
& e^{\beta(h-\delta)+\bar{\beta}(\bar{h}-\bar{\delta})} \int \mathrm{d} F\left(h^{\prime}, \bar{h}^{\prime}\right) e^{-\beta h^{\prime}-\bar{\beta} \bar{h}^{\prime}} \Phi_{-}\left(h^{\prime}, \bar{h}^{\prime}\right) \\
& \leq \int_{h-\delta}^{h+\delta} \int_{\bar{h}-\bar{\delta}}^{\bar{h}+\bar{\delta}} \mathrm{d} F\left(h, \bar{h}^{\prime}\right) \leq \\
& e^{\beta(h+\delta)+\bar{\beta}(\bar{h}+\bar{\delta})} \int \mathrm{d} F\left(h^{\prime}, \bar{h}^{\prime}\right) e^{-\beta h^{\prime}-\bar{\beta} \bar{h}^{\prime}} \Phi_{+}\left(h^{\prime}, \bar{h}^{\prime}\right) .
\end{aligned}
$$

At this point, we use the Fourier transform $\hat{\Phi}_{ \pm}(t, \bar{t})$, defined as

$$
\Phi_{ \pm}\left(h^{\prime}, \bar{h}^{\prime}\right) \equiv \int_{-\infty}^{\infty} \mathrm{d} t \int_{-\infty}^{\infty} \mathrm{d} \bar{t} \hat{\Phi}_{ \pm}(t, \bar{t}) e^{-i h^{\prime} t-i \bar{h}^{\prime} \bar{t}}
$$

This facilitates us to rewrite the inequality in (3.4) as

$$
\begin{aligned}
& \int \mathrm{d} h^{\prime} \mathrm{d} \bar{h}^{\prime} \mathcal{L}_{\rho}(\beta+i t, \bar{\beta}+i \bar{t}) \hat{\Phi}_{-}(t, \bar{t}) \\
& \leq \int_{h-\delta}^{h+\delta} \int_{\bar{h}-\bar{\delta}}^{\bar{h}+\bar{\delta}} \mathrm{d} F\left(h, \bar{h}^{\prime}\right) \leq \\
& \int \mathrm{d} h^{\prime} \mathrm{d} \bar{h}^{\prime} \mathcal{L}_{\rho}(\beta+i t, \bar{\beta}+i \bar{t}) \hat{\Phi}_{+}(t, \bar{t}),
\end{aligned}
$$

where we have

$$
\mathcal{L}_{\rho}(\beta, \bar{\beta}) \equiv \int_{0}^{\infty} \mathrm{d} h \int_{0}^{\infty} \mathrm{d} \bar{h} \rho(h, \bar{h}) e^{-\beta h-\bar{\beta} \bar{h}}
$$

Next we need to split $Z=Z_{L}+Z_{H}$. In [19], for the mixed temperature analysis, the light sector is chosen to be $\left\{(h, \bar{h}) \mid h<\frac{c}{24}\right.$ or $\left.\bar{h}<\frac{c}{24}\right\}$ and the heavy sector is the complement of that. Here, we choose a different light sector $\left\{(h, \bar{h}) \mid h+\bar{h}<\frac{c}{12}\right\}$ which has finite size (for large central charge, this is not true and one needs to have an extra sparseness condition on the low lying spectra $[2,19])$. So at least, in principle, we can choose $\rho_{*}(h, \bar{h})$ such that $\rho_{*}$ reproduce the contributions from all operators in the light sector. In practice, we take $\rho_{*}$ such that it reproduces only the vacuum state contribution, that is,

$$
\rho_{*}(h, \bar{h})=\left[\pi \sqrt{\frac{c}{6}} \frac{I_{1}\left(2 \pi \sqrt{\frac{c}{6}\left(h-\frac{c}{24}\right)}\right)}{\sqrt{h-\frac{c}{24}}} \theta\left(h-\frac{c}{24}\right)+\delta\left(h-\frac{c}{24}\right)\right] \times(h \rightarrow \bar{h}),
$$

which has the asymptotic behavior

$$
\rho_{*}(h, \bar{h}) \sim \sqrt{\frac{c}{96}}\left(\frac{1}{h^{3} \bar{h}^{3}}\right)^{1 / 4} e^{2 \pi \sqrt{\frac{c h}{6}}+2 \pi \sqrt{\frac{c \bar{h}}{6}}}\left[1+O\left(h^{-1 / 2}\right)+O\left(\bar{h}^{-1 / 2}\right)\right] .
$$


Then we can write $e^{-(\beta+i t+\bar{\beta}+i \bar{t}) c / 24} Z_{L}\left(\frac{4 \pi^{2}}{\beta+i t}, \frac{4 \pi^{2}}{\beta+i \bar{t}}\right)=\mathcal{L}_{\rho_{*}, L}(\beta+i t, \bar{\beta}+i \bar{t})$. So we get

$$
\begin{aligned}
& e^{\beta(h-\delta)+\bar{\beta}(\bar{h}-\bar{\delta})}\left(\int_{-\infty}^{\infty} \mathrm{d} t \mathrm{~d} \bar{t} \hat{\Phi}_{-}(t, \bar{t}) \mathcal{L}_{\rho_{*}, L}(\beta+i t, \bar{\beta}+i \bar{t})\right. \\
& \left.-\left|\int_{-\infty}^{\infty} \mathrm{d} t \mathrm{~d} \bar{t} e^{-(\beta+i t+\bar{\beta}+i \bar{t}) c / 24} \hat{\Phi}_{-}(t, \bar{t}) Z_{H}\left(\frac{4 \pi^{2}}{\beta+i t}, \frac{4 \pi^{2}}{\bar{\beta}+i \bar{t}}\right)\right|\right) \\
& \leq \int_{h-\delta}^{h+\delta} \int_{\bar{h}-\bar{\delta}}^{\bar{h}+\bar{\delta}} \mathrm{d} F\left(h, \bar{h}^{\prime}\right) \leq \\
& e^{\beta(h+\delta)+\bar{\beta}(\bar{h}+\bar{\delta})}\left(\int_{-\infty}^{\infty} \mathrm{d} t \mathrm{~d} \bar{t} \hat{\Phi}_{+}(t, \bar{t}) \mathcal{L}_{\rho_{*}, L}(\beta+i t, \bar{\beta}+i \bar{t})\right. \\
& \left.\quad+\left|\int_{-\infty}^{\infty} \mathrm{d} t \mathrm{~d} \bar{t} e^{-(\beta+i t+\bar{\beta}+i \bar{t}) c / 24} \hat{\Phi}_{+}(t, \bar{t}) Z_{H}\left(\frac{4 \pi^{2}}{\beta+i t}, \frac{4 \pi^{2}}{\bar{\beta}+i \bar{t}}\right)\right|\right),
\end{aligned}
$$

Now we can estimate the contribution from the heavy sector using the HKS bound. We choose $\Phi_{ \pm}$such that $\hat{\Phi}_{ \pm}(t, \bar{t})$ have finite support $\left[-\Lambda_{ \pm}, \Lambda_{ \pm}\right]$for $t$ and $\bar{t}$. A possible choice can be made via modifying the functions appearing in $[2,3]$ a little bit. To be concrete, let us make the following choices:

$$
\begin{aligned}
& \Phi_{+}\left(h^{\prime}, \bar{h}^{\prime}\right)=\frac{f_{+}\left(h-h^{\prime}\right) f_{+}\left(\bar{h}-\bar{h}^{\prime}\right)}{f_{+}(\delta) f_{+}(\bar{\delta})}, \\
& \Phi_{-}\left(h^{\prime}, \bar{h}^{\prime}\right)=f_{-}\left(h-h^{\prime}\right) f_{-}\left(\bar{h}-\bar{h}^{\prime}\right)\left(1-\left(\frac{h-h^{\prime}}{\delta}\right)^{2}-\left(\frac{\bar{h}-\bar{h}^{\prime}}{\bar{\delta}}\right)^{2}\right),
\end{aligned}
$$

where we have

$$
f_{ \pm}(x)=\left[\operatorname{sinc}\left(\frac{\Lambda_{ \pm} x}{4}\right)\right]^{4}
$$

We remark that for $\Phi_{-}$, the locus $1-(a / \delta)^{2}-(b / \bar{\delta})^{2}=0$ is inside the rectangle region, hence it is a valid choice conforming to the inequality (3.1). Here $a=h-h^{\prime}$ and $b=\bar{h}-\bar{h}^{\prime}$. At this point, our aim is to show that

$$
I_{ \pm}=e^{\beta(h \pm \delta)+\bar{\beta}(\bar{h} \pm \bar{\delta})}\left|\int_{-\infty}^{\infty} \mathrm{d} t \mathrm{~d} \bar{t} e^{-(\beta+i t+\bar{\beta}+i \bar{t}) c / 24} \hat{\Phi}_{ \pm}(t, \bar{t}) Z_{H}\left(\frac{4 \pi^{2}}{\beta+i t}, \frac{4 \pi^{2}}{\bar{\beta}+i \bar{t}}\right)\right|
$$

is sub-leading. We will make use of the most basic HKS bound [19] for $\Delta$ in a clever way. We remark that by requiring the saddle of the light sector is located at $(h, \bar{h})$, we find $\beta=\pi \sqrt{\frac{c}{6 h}} \ll 1, \bar{\beta}=\pi \sqrt{\frac{c}{6 h}} \ll 1$, so some terms such as $\beta \delta$ can be dropped from the bounds as it goes to 0 for large $h, \bar{h}$. Then using the fact that $\Phi_{ \pm}$is a bandlimited function, we have

$$
I_{ \pm} \leq e^{\beta h+\bar{\beta} \bar{h}} \int \mathrm{d} t \mathrm{~d} \bar{t} Z_{H}\left(\frac{4 \pi^{2} \beta}{\beta^{2}+t^{2}}, \frac{4 \pi^{2} \bar{\beta}}{\bar{\beta}^{2}+\bar{t}^{2}}\right)\left|\hat{\Phi}_{ \pm}(t, \bar{t})\right| .
$$

Now $Z_{H}$ has contribution from states where either $h^{\prime}$ or $\bar{h}^{\prime}$ is greater than $c / 24$. Since the contributing states have $h^{\prime}+\bar{h}^{\prime}>c / 12$, both can not be less than or equal to $\frac{c}{24}$. We 
illustrate the case for $h^{\prime}>c / 24$.

$$
\begin{aligned}
Z_{H} \ni & \exp \left[-\frac{4 \pi^{2} \beta}{\beta^{2}+t^{2}}\left(h^{\prime}-\frac{c}{24}\right)-\frac{4 \pi^{2} \bar{\beta}}{\bar{\beta}^{2}+\bar{t}^{2}}\left(\bar{h}^{\prime}-\frac{c}{24}\right)\right] \\
& \leq e^{\frac{\pi^{2} c}{6 \beta}} \exp \left[-\frac{4 \pi^{2} \beta}{\beta^{2}+\Lambda_{ \pm}^{2}}\left(h^{\prime}-\frac{c}{24}\right)-\frac{4 \pi^{2} \bar{\beta}}{\bar{\beta}^{2}+\Lambda_{ \pm}^{2}} \bar{h}^{\prime}\right] \\
& \leq e^{\frac{\pi^{2} c}{6 \beta}} e^{-\frac{\pi^{2} \beta_{*} c}{6\left(\beta_{*}^{2}+\Lambda_{ \pm}^{2}\right)}} \exp \left[-\frac{4 \pi^{2} \beta_{*}}{\beta_{*}^{2}+\Lambda_{ \pm}^{2}}\left(h^{\prime}+\bar{h}^{\prime}-\frac{c}{12}\right)\right]
\end{aligned}
$$

where $\beta_{*}$ is defined as $\beta_{*}=\min (\beta, \bar{\beta})$, hence we have (for small enough $\beta$ and $\bar{\beta}$ )

$$
\min \left(\frac{4 \pi^{2} \beta}{\beta^{2}+\Lambda_{ \pm}^{2}}, \frac{4 \pi^{2} \bar{\beta}}{\bar{\beta}^{2}+\Lambda_{ \pm}^{2}}\right)=\frac{4 \pi^{2} \beta_{*}}{\beta_{*}^{2}+\Lambda_{ \pm}^{2}},
$$

Thus we have

$$
I_{ \pm} \leq e^{\beta h+\bar{\beta} \bar{h}}\left(e^{\frac{\pi^{2} c}{6 \bar{\beta}}}+e^{\frac{\pi^{2} c}{6 \beta}}\right) e^{-\frac{\pi^{2} \beta_{*} c}{6\left(\beta_{*}^{2}+\Lambda_{ \pm}^{2}\right)}} Z_{H, \Delta}\left(\frac{4 \pi^{2} \beta_{*}}{\beta_{*}^{2}+\Lambda_{ \pm}^{2}}\right)
$$

where the last $Z_{H, \Delta}=\sum_{\Delta>c / 12} e^{-\beta(\Delta-c / 12)}$ is the heavy contribution from the original HKS bound for $\Delta$. We will be showing that the above term is subleading. There are two pieces, one with $e^{\frac{\pi^{2} c}{6 \beta}}$ and another with $e^{\frac{\pi^{2} c}{6 \beta}}$. Let us illustrate the subleading nature of the term with $e^{\frac{\pi^{2} c}{6 \bar{\beta}}}$. The other term can be treated similarly.

In $\beta, \bar{\beta} \rightarrow 0$ limit, we have

$$
\begin{gathered}
e^{\beta h+\bar{\beta} \bar{h}} e^{\frac{\pi^{2} c}{6 \bar{\beta}}} e^{-\frac{\pi^{2} \beta_{* c}}{6\left(\beta_{*}^{2}+\Lambda_{ \pm}^{2}\right)}} Z_{H, \Delta}\left(\frac{4 \pi^{2} \beta_{*}}{\beta_{*}^{2}+\Lambda_{ \pm}^{2}}\right) \leq e^{\beta h+\bar{\beta} \bar{h}} e^{\frac{\pi^{2} c}{6 \beta}} Z_{H, \Delta}\left(\frac{4 \pi^{2} \beta_{*}}{\beta_{*}^{2}+\Lambda_{ \pm}^{2}}\right) \\
\sim e^{\pi \sqrt{\frac{c h}{6}}+2 \pi \sqrt{\frac{c \bar{h}}{6}}+2 \pi \sqrt{\frac{c h *}{6}}\left(\frac{\Lambda_{ \pm}}{2 \pi}\right)^{2}} \\
\leq\left\{\begin{array}{l}
h \geq \bar{h}: e^{2 \pi \sqrt{\frac{c h}{6}}+2 \pi \sqrt{\frac{c \bar{h}}{6}}+2 \pi \sqrt{\frac{c h}{6}}\left(\left(\frac{\Lambda_{ \pm}}{2 \pi}\right)^{2}-\frac{1}{2}\right)} \\
h<\bar{h}: e^{\pi \sqrt{\frac{c h}{6}}+2 \pi \sqrt{\frac{c \bar{h}}{6}}+2 \pi \sqrt{\frac{c \bar{h}}{6}}\left(\frac{\Lambda_{ \pm}}{2 \pi}\right)^{2}} .
\end{array}\right.
\end{gathered}
$$

where $h^{*}=\max (h, \bar{h})$ and $\tau=2 \min (h, \bar{h})$. To make the contribution from the heavy sector sub-leading, we need

$$
\Lambda_{ \pm}<\min \left(\frac{2 \pi}{\sqrt{2}}, \frac{2 \pi}{\sqrt{2} \gamma}\right)
$$

where $\frac{\tau}{2} \gamma^{4} \simeq h^{*}$ (clearly, $\gamma>1$ ). This can be achieved by choosing

$$
\Lambda_{ \pm}<\frac{\sqrt{2} \pi}{\gamma}
$$

Then at large $h, \bar{h}$, we have the following bound, starting from (3.10) (the second term i.e. the term with the absolute value in (3.10) has already been shown to be subleading 
and we rewrite the first term as an integral over $h^{\prime}, \bar{h}^{\prime}$ below):

$$
\begin{aligned}
& e^{\beta(h-\delta)+\bar{\beta}(\bar{h}-\bar{\delta})} \int \mathrm{d} h^{\prime} \mathrm{d} \bar{h}^{\prime} \rho_{*}\left(h^{\prime}, \bar{h}^{\prime}\right) \Phi_{-}\left(h^{\prime}, \bar{h}^{\prime}\right) e^{-\beta h^{\prime}-\bar{\beta} \bar{h}^{\prime}} \\
& \leq \int_{h-\delta}^{h+\delta} \int_{\bar{h}-\bar{\delta}}^{\bar{h}+\bar{\delta}} \mathrm{d} F\left(h, \bar{h}^{\prime}\right) \leq \\
& e^{\beta(h+\delta)+\bar{\beta}(\bar{h}+\bar{\delta})} \int \mathrm{d} h^{\prime} \mathrm{d} \bar{h}^{\prime} \rho_{*}\left(h^{\prime}, \bar{h}^{\prime}\right) \Phi_{+}\left(h^{\prime}, \bar{h}^{\prime}\right) e^{-\beta h^{\prime}-\bar{\beta} \bar{h}^{\prime}} .
\end{aligned}
$$

We can evaluate this integral by saddle point approximation,

$$
c_{-} \rho_{*}(h, \bar{h}) \leq \frac{1}{4 \delta \bar{\delta}} \int_{h-\delta}^{h+\delta} \int_{\bar{h}-\bar{\delta}}^{\bar{h}+\bar{\delta}} \mathrm{d} F\left(h, \bar{h}^{\prime}\right) \leq c_{+} \rho_{*}(h, \bar{h}),
$$

where $c_{ \pm}$is defined as

$$
c_{ \pm}=\frac{1}{4} \int \mathrm{d} x \mathrm{~d} y \Phi_{ \pm}(h+\delta x, \bar{h}+\bar{\delta} y) .
$$

With the previous choice of $\Phi_{ \pm}$, we have

$$
\begin{aligned}
& c_{+}=\frac{16 \pi^{2}}{9} \frac{1}{\delta \bar{\delta} \Lambda_{+}^{2} \operatorname{sinc}^{4}\left(\delta \Lambda_{+} / 4\right) \operatorname{sinc}^{4}\left(\bar{\delta} \Lambda_{+} / 4\right)}, \\
& c_{-}=\frac{16 \pi^{2}}{9} \frac{\delta^{2} \bar{\delta}^{2} \Lambda_{-}^{2}-12 \delta^{2}-12 \bar{\delta}^{2}}{\delta^{3} \bar{\delta}^{3} \Lambda_{-}^{4}} .
\end{aligned}
$$

where we must optimize over $0<\Lambda_{ \pm}<\frac{\sqrt{2} \pi}{\gamma}$ to get the tightest bound while keeping $\delta, \bar{\delta}$ arbitrary.

The condition for the lower bound to be positive is

$$
\frac{1}{\delta^{2}}+\frac{1}{\bar{\delta}^{2}}<\frac{\Lambda_{-}^{2}}{12}
$$

The allowed region increases as we increase $\Lambda_{-}$. And the minimum area such that there has to be at least one operator is given by $4 \delta \bar{\delta}=\frac{48 \gamma^{2}}{\pi^{2}}=4.86 \gamma^{2}$ at $\delta=\bar{\delta}=\frac{2 \sqrt{3} \gamma}{\pi}$. This analysis can be made sensitive to primaries only, thus gives an asymptotic gap between primaries. We suspect that the above does not give the tightest bound for spectral gap (this intuition is coming from the similar analysis done for the spectral gap in $\Delta$, appearing in $[2,3])$ !

Next we can keep $\delta, \bar{\delta}$ arbitrary and optimize over $0<\Lambda_{ \pm}<\frac{\sqrt{2} \pi}{\gamma}$ to get the tightest bound.

(a) For lower bound:

$$
\begin{aligned}
\frac{1}{4 \delta \bar{\delta}} \int_{h-\delta}^{h+\delta} \int_{\bar{h}-\bar{\delta}}^{\bar{h}+\bar{\delta}} \mathrm{d} F\left(h, \bar{h}^{\prime}\right) \geq \frac{\pi^{2}}{27} \frac{\delta \bar{\delta}}{\delta^{2}+\bar{\delta}^{2}} \rho_{*}(h, \bar{h}), & \frac{\sqrt{\delta^{2}+\bar{\delta}^{2}}}{\delta \bar{\delta}}<\frac{\pi}{2 \sqrt{3} \gamma}, \\
\frac{1}{4 \delta \bar{\delta}} \int_{h-\delta}^{h+\delta} \int_{\bar{h}-\bar{\delta}}^{\bar{h}+\bar{\delta}} \mathrm{d} F\left(h, \bar{h}^{\prime}\right) \geq \frac{8 \pi^{2} \gamma^{2} \delta^{2} \bar{\delta}^{2}-48 \gamma^{4}\left(\delta^{2}+\bar{\delta}^{2}\right)}{9 \pi^{2} \delta^{3} \bar{\delta}^{3}} \rho_{*}(h, \bar{h}), & \frac{\sqrt{\delta^{2}+\bar{\delta}^{2}}}{\delta \bar{\delta}} \geq \frac{\pi}{2 \sqrt{3} \gamma} .
\end{aligned}
$$


(b) For upper bound:

$$
\begin{array}{lll}
\frac{1}{4 \delta \bar{\delta}} \int_{h-\delta}^{h+\delta} \int_{\bar{h}-\bar{\delta}}^{\bar{h}+\bar{\delta}} \mathrm{d} F\left(h, \bar{h}^{\prime}\right) \leq \frac{16 \pi^{2}}{9 \delta \bar{\delta}\left(\Lambda_{+}^{*}\right)^{2}} \frac{\rho_{*}(h, \bar{h})}{\operatorname{sinc}\left(\frac{\delta \Lambda_{+}^{*}}{4}\right)^{4} \operatorname{sinc}\left(\frac{\bar{\delta} \Lambda_{+}^{*}}{4}\right)^{4}}, & \Lambda_{+}^{*} \leq \frac{\sqrt{2} \pi}{\gamma}, \\
\frac{1}{4 \delta \bar{\delta}} \int_{h-\delta}^{h+\delta} \int_{\bar{h}-\bar{\delta}}^{\bar{h}+\bar{\delta}} \mathrm{d} F\left(h, \bar{h}^{\prime}\right) \leq \frac{8 \gamma^{2}}{9 \delta \bar{\delta}} \frac{\rho_{*}(h, \bar{h})}{\operatorname{sinc}\left(\frac{\delta \sqrt{2} \pi}{4 \gamma}\right)^{4} \operatorname{sinc}\left(\frac{\bar{\delta} \sqrt{2} \pi}{4 \gamma}\right)^{4}}, & \Lambda_{+}^{*}>\frac{\sqrt{2} \pi}{\gamma} .
\end{array}
$$

where $\Lambda_{+}^{*}(\delta, \bar{\delta})$ is the non-zero least positive solution of

$$
\delta \Lambda_{+} \cot \left(\frac{\delta \Lambda_{+}}{4}\right)+\bar{\delta} \Lambda_{+} \cot \left(\frac{\bar{\delta} \Lambda_{+}}{4}\right)=6 .
$$

\subsection{Analysis of $O(1)$ window assuming a twist gap}

We have seen that the result proven in the previous subsection holds true when $h$ and $\bar{h}$ are of the same order asymptotically. When we make the analysis sensitive to primary, this feature persists. Nonetheless, we can circumnavigate this issue by assuming an existence of twist gap (as defined in 1.1). One can also do this for the analysis sensitive to all the operators. The only catch is that one has to separately treat the zero twist operators with dimension greater than $c / 12$. We revisit the analysis of suppression of heavy region in the light of the above discussion. At first, we take up the analysis for all the operators.

We go back to the eq. (3.16) and redo the first part of the analysis. We separate out $Z_{H}$ into two pieces, one with zero twist heavy operators, we name it $Z_{H}^{(0)}$, while the other one contains all the heavy operators with non-zero twist, we name it $Z_{H}^{(\tau)}$. We start with analyzing $Z_{H}^{(\tau)}$, assuming a twist gap. We have two scenarios.

Scenario I: if $g \leq \frac{c}{12}$, we have

$$
\begin{aligned}
Z_{H}^{(\tau)} \ni & \exp \left[-\frac{4 \pi^{2} \beta}{\beta^{2}+t^{2}}\left(h^{\prime}-\frac{c}{24}\right)-\frac{4 \pi^{2} \bar{\beta}}{\bar{\beta}^{2}+\bar{t}^{2}}\left(\bar{h}^{\prime}-\frac{c}{24}\right)\right] \\
& \leq e^{\frac{\pi^{2} c\left(1-\frac{12 g}{c}\right)}{6 \bar{\beta}}+\frac{\pi^{2} c\left(1-\frac{12 g}{c}\right)}{6 \beta}} \exp \left[-\frac{4 \pi^{2} \beta}{\beta^{2}+\Lambda_{ \pm}^{2}}\left(h^{\prime}-\frac{g}{2}\right)-\frac{4 \pi^{2} \bar{\beta}}{\bar{\beta}^{2}+\Lambda_{ \pm}^{2}}\left(\bar{h}^{\prime}-\frac{g}{2}\right)\right] \\
& \leq e^{\frac{\pi^{2} c\left(1-\frac{12 g}{c}\right)}{6 \bar{\beta}}+\frac{\pi^{2} c\left(1-\frac{12 g}{c}\right)}{6 \beta}} \exp \left[-\frac{4 \pi^{2} \beta_{*}}{\beta_{*}^{2}+\Lambda_{ \pm}^{2}}\left(h^{\prime}+\bar{h}^{\prime}-g\right)\right] \\
& \leq e^{\frac{\pi^{2} c\left(1-\frac{12 g}{c}\right)\left(\frac{1}{\beta}+\frac{1}{\beta}\right)}{6}} \exp \left[-\frac{4 \pi^{2} \beta_{*}}{\beta_{*}^{2}+\Lambda_{ \pm}^{2}}\left(h^{\prime}+\bar{h}^{\prime}-\frac{c}{12}\right)\right] .
\end{aligned}
$$

Here going from the first inequality to the second one, we used $2 h^{\prime}>g, 2 \bar{h}^{\prime}>g$; going from the second one to the third (last) one, we used $g \leq c / 12$. The rest of the analysis goes in a similar manner, as done subsequently after (3.16) and we deduce:

$$
\Lambda_{ \pm}<\frac{\sqrt{2} \pi}{\sigma}, \quad \sigma^{2} \equiv\left(\frac{c}{12 g}\right)
$$


Scenario II: if $g \geq \frac{c}{12}$, we have $h^{\prime}>c / 24$ and $\bar{h}^{\prime}>c / 24$, leading to

$$
\begin{aligned}
Z_{H}^{(\tau)} & \ni \exp \left[-\frac{4 \pi^{2} \beta}{\beta^{2}+t^{2}}\left(h^{\prime}-\frac{c}{24}\right)-\frac{4 \pi^{2} \bar{\beta}}{\bar{\beta}^{2}+\bar{t}^{2}}\left(\bar{h}^{\prime}-\frac{c}{24}\right)\right] \\
& \leq \exp \left[-\frac{4 \pi^{2} \beta_{*}}{\beta_{*}^{2}+\Lambda_{ \pm}^{2}}\left(h^{\prime}+\bar{h}^{\prime}-\frac{c}{12}\right)\right] .
\end{aligned}
$$

The rest of the analysis goes in a similar manner and we deduce:

$$
\Lambda_{ \pm}<\sqrt{2} \pi \text {. }
$$

Now we come back to analyzing the zero twist heavy sector. For this sector, $h^{*}=$ $\max (h, \bar{h})=\Delta>c / 12>c / 24$, thus we have

$$
\begin{aligned}
& Z_{H}^{(0)} \\
& =\sum_{h^{\prime}} \exp \left[-\frac{4 \pi^{2} \beta}{\beta^{2}+t^{2}}\left(h^{\prime}-\frac{c}{24}\right)+\frac{\pi^{2} \bar{\beta} c}{6\left(\bar{\beta}^{2}+\bar{t}^{2}\right)}\right]+\sum_{\bar{h}^{\prime}} \exp \left[\frac{\pi^{2} \beta c}{6\left(\beta^{2}+t^{2}\right)}-\frac{4 \pi^{2} \bar{\beta}}{\bar{\beta}^{2}+\bar{t}^{2}}\left(\bar{h}^{\prime}-\frac{c}{24}\right)\right] \\
& \leq \sum_{h^{\prime}} \exp \left[-\frac{4 \pi^{2} \beta}{\beta^{2}+\Lambda^{2}}\left(\Delta_{h^{\prime}, 0}-\frac{c}{24}\right)+\frac{\pi^{2} c}{6 \bar{\beta}}\right]+\sum_{\bar{h}^{\prime}} \exp \left[\frac{\pi^{2} c}{6 \beta}-\frac{4 \pi^{2} \bar{\beta}}{\bar{\beta}^{2}+\Lambda^{2}}\left(\Delta_{0, \bar{h}^{\prime}}-\frac{c}{24}\right)\right] \\
& \leq e^{\frac{\pi^{2} c}{6 \bar{\beta}}} \sum_{h^{\prime}} \exp \left[-\frac{4 \pi^{2} \beta}{\beta^{2}+\Lambda^{2}}\left(\Delta_{h^{\prime}, 0}-\frac{c}{24}\right)\right]+e^{\frac{\pi^{2} c}{6 \beta}} \sum_{\bar{h}^{\prime}} \exp \left[-\frac{4 \pi^{2} \bar{\beta}}{\bar{\beta}^{2}+\Lambda_{ \pm}^{2}}\left(\Delta_{0, \bar{h}^{\prime}}-\frac{c}{24}\right)\right] \\
& \leq e^{\frac{\pi^{2} c}{6 \bar{\beta}}} \sum_{h^{\prime}} \exp \left[-\frac{4 \pi^{2} \beta}{\beta^{2}+\Lambda_{ \pm}^{2}}\left(\Delta_{h^{\prime}, 0}-\frac{c}{12}\right)\right]+e^{\frac{\pi^{2} c}{6 \beta}} \sum_{\bar{h}^{\prime}} \exp \left[-\frac{4 \pi^{2} \bar{\beta}}{\bar{\beta}^{2}+\Lambda_{ \pm}^{2}}\left(\Delta_{0, \bar{h}^{\prime}}-\frac{c}{12}\right)\right] \\
& \leq e^{\frac{\pi^{2} c}{6 \bar{\beta}}} \sum_{h^{\prime}} \exp \left[-\frac{4 \pi^{2} \beta}{\beta^{2}+\Lambda_{ \pm}^{2}}\left(\Delta_{h^{\prime}, 0}-\frac{c}{12}\right)\right]+e^{\frac{\pi^{2} c}{6 \beta}} \sum_{\bar{h}^{\prime}} \exp \left[-\frac{4 \pi^{2} \bar{\beta}}{\bar{\beta}^{2}+\Lambda_{ \pm}^{2}}\left(\Delta_{0, \bar{h}^{\prime}}-\frac{c}{12}\right)\right] \\
& \leq e^{\frac{\pi^{2} c}{6 \beta}} \sum_{\Delta} \exp \left[-\frac{4 \pi^{2} \beta}{\beta^{2}+\Lambda_{ \pm}^{2}}\left(\Delta-\frac{c}{12}\right)\right]+\sum_{\Delta} e^{\frac{\pi^{2} c}{6 \beta}} \exp \left[-\frac{4 \pi^{2} \bar{\beta}}{\bar{\beta}^{2}+\Lambda_{ \pm}^{2}}\left(\Delta-\frac{c}{12}\right)\right] \\
& \leq e^{\pi \sqrt{\frac{c \bar{h}}{6}}} Z_{H, \Delta}\left(\frac{4 \pi^{2} \beta}{\beta^{2}+\Lambda_{ \pm}^{2}}\right)+e^{\pi \sqrt{\frac{c h}{6}}} Z_{H, \Delta}\left(\frac{4 \pi^{2} \bar{\beta}}{\bar{\beta}^{2}+\Lambda_{ \pm}^{2}}\right) \cdot
\end{aligned}
$$

The subscript on $\Delta$ in the second line denotes the actual conformal weights of the operator and in the penultimate line, we have extended the sum to all the heavy operators. Now one can see the zero twist heavy sector is suppressed as long as we choose $\Lambda_{ \pm}<\sqrt{2} \pi$. Thus, combining everything, we have

$$
\Lambda_{ \pm}< \begin{cases}\min \left(\sqrt{2} \pi, \frac{\sqrt{2} \pi}{\zeta}\right)=\frac{\sqrt{2} \pi}{\zeta}, & g \leq c / 12 \\ \sqrt{2} \pi, & g \geq c / 12\end{cases}
$$

where $\zeta^{2}=\frac{c}{12 g}$. We can combine the above to write for all $g$,

$$
\Lambda_{ \pm}<\min \left(\sqrt{2} \pi, \frac{\sqrt{2} \pi}{\zeta}\right) .
$$


The above has immediate implication in terms of asymptotic gap for all the operators, in particular, the gap does not depend on the term $\gamma$ anymore. Nonetheless, as we already know existence of descendants asymptotically, the asymptotic gap for all the operators is not so illuminating, so we will not illustrate upon this. Rather we come back to this when we make our analysis sensitive to primaries and in that scenario, the result about asymptotic gap is indeed illuminating.

Analysis for primaries: twist gap complementary to asymptotic spectral gap. The analysis for primaries proceeds in a similar manner. We will be estimating the following object

$$
\exp \left[S_{\delta, \bar{\delta}}^{\mathrm{Vir}}\right]=\int_{\bar{h}-\bar{\delta}}^{\bar{h}+\bar{\delta}} \int_{h-\delta}^{h+\delta} \mathrm{d} h^{\prime} \mathrm{d} \bar{h}^{\prime} \rho^{\mathrm{Vir}}\left(h^{\prime}, \bar{h}^{\prime}\right)
$$

where $\rho^{\operatorname{Vir}}\left(h^{\prime}, \bar{h}^{\prime}\right)$ is the density of primaries. Instead of the partition function we consider the following object (for $c>1$, the expansion of this object is universal)

$$
\begin{aligned}
& Z_{\text {primary }}(\beta, \bar{\beta}) \\
& \equiv \eta(\beta) \eta(\bar{\beta}) Z(\beta)=e^{\beta \frac{c-1}{24}+\bar{\beta} \frac{c-1}{24}}\left[\left(1-e^{-\beta}\right)\left(1-e^{-\bar{\beta}}\right)+\sum_{h^{\prime} \neq 0, \bar{h}^{\prime} \neq 0} d_{h^{\prime}, \bar{h}^{\prime}} e^{-\beta h^{\prime}-\bar{\beta} \bar{h}^{\prime}}\right] .
\end{aligned}
$$

Under modular transformation, we have

$$
Z_{\text {primary }}(\beta, \bar{\beta})=\sqrt{\frac{2 \pi}{\beta}} \sqrt{\frac{2 \pi}{\bar{\beta}}} Z_{\text {primary }}\left(\frac{4 \pi^{2}}{\beta}, \frac{4 \pi^{2}}{\bar{\beta}}\right) .
$$

Then we define the crossing $\rho_{*}^{\operatorname{Vir}}\left(h^{\prime}, \bar{h}^{\prime}\right)=\rho_{*}^{\operatorname{Vir}}\left(h^{\prime}\right) \rho_{*}^{\operatorname{Vir}}\left(\bar{h}^{\prime}\right)$ to reproduce the high temperature behavior of $Z_{\text {primary }}(\beta, \bar{\beta})$ i.e. we have

$$
\begin{aligned}
& \int_{0}^{\infty} \mathrm{d} h^{\prime} e^{-\beta\left(h^{\prime}-\frac{c-1}{24}\right)} \rho_{*}^{\operatorname{Vir}}\left(h^{\prime}\right)=\sqrt{\frac{2 \pi}{\beta}}\left(\exp \left[\frac{\pi^{2}(c-1)}{6 \beta}\right]-\exp \left[\frac{\pi^{2}(c-25)}{6 \beta}\right]\right), \\
& \int_{0}^{\infty} \mathrm{d} \bar{h}^{\prime} e^{-\bar{\beta}\left(\bar{h}^{\prime}-\frac{c-1}{24}\right)} \rho_{*}^{\operatorname{Vir}}\left(\bar{h}^{\prime}\right)=\sqrt{\frac{2 \pi}{\bar{\beta}}}\left(\exp \left[\frac{\pi^{2}(c-1)}{6 \beta}\right]-\exp \left[\frac{\pi^{2}(c-25)}{6 \bar{\beta}}\right]\right) .
\end{aligned}
$$

Explicitly, $\rho_{*}^{\text {Vir }}$ would be given by the following function:

$$
\rho_{*}^{\operatorname{Vir}}\left(h^{\prime}\right)=\left\{\begin{array}{l}
0 \text { if } h^{\prime}<\frac{c-1}{24} \\
\frac{\sqrt{2}}{\sqrt{h-\frac{c-1}{24}}}\left[\cosh \left(4 \pi \sqrt{\frac{(c-1)}{24}\left(h-\frac{c-1}{24}\right)}\right)-\cosh \left(4 \pi \sqrt{\frac{(c-25)}{24}\left(h-\frac{c-1}{24}\right)}\right)\right] .
\end{array}\right.
$$

The analysis pertaining to the estimation of the heavy part presented before for the analysis of all the operators can be used as a stepping stone for a similar analysis for primaries for 
$c>1$ CFTs. We again use bandlimited functions and we deduce that the support $\Lambda_{ \pm}$has to satisfy: ${ }^{7}$

$$
\Lambda_{ \pm}<\min \left(\frac{\sqrt{2} \pi}{\zeta_{p}}, \sqrt{2} \pi\right), \quad \zeta_{p}^{2} \equiv\left(\frac{c-1}{12 g}\right) .
$$

The leading answer comes out to be

$$
\begin{aligned}
& \frac{1}{2} \frac{c_{-}}{\sqrt{h-\frac{c-1}{24}} \sqrt{\bar{h}-\frac{c-1}{24}}} \exp \left[2 \pi\left(\sqrt{\frac{(c-1) h}{6}}+\sqrt{\frac{(c-1) \bar{h}}{6}}\right)\right] \\
& \leq \frac{1}{4 \delta \bar{\delta}} \exp \left[S_{\delta, \bar{\delta}}^{\mathrm{Vir}}\right] \leq \\
& \frac{1}{2} \frac{c_{+}}{\sqrt{h-\frac{c-1}{24}} \sqrt{\bar{h}-\frac{c-1}{24}}} \exp \left[2 \pi\left(\sqrt{\frac{(c-1) h}{6}}+\sqrt{\frac{(c-1) \bar{h}}{6}}\right)\right],
\end{aligned}
$$

where $c_{ \pm}$is defines as in the eq. (3.24).

Asymptotic gap. Now we come back to our discussion of asymptotic gap of primaries. We use the function given in eq. (3.12) but now with constraint as given in eq. (3.42). Thus the asymptotic binding square will have length $\frac{4 \sqrt{3} \sigma}{\pi}$ and the binding circle would have radius $\frac{r \sigma}{\sqrt{2}}+\epsilon_{g}$ with $\epsilon_{g}>0$, and $\sigma, r$ are given by

$$
\sigma=\max \left(1, \frac{c-1}{12 g}\right), \quad r=\frac{4 \sqrt{3}}{\pi} .
$$

If we consider tensoring the chiral Monster CFT with its antichiral avatar, we find $g=4$ and our result predicts that the asymptotic spectral gap involves a circle of radius $\frac{2 \sqrt{6}}{\pi}$ irrespective of how infinity is approached. This is above the suspected optimal value 1 (see figure 4). In a unitary compact CFT without conserved currents, there is a bound on twist gap [20]:

$$
g \leq \frac{c-1}{12}
$$

In that scenario, we have

$$
\Lambda_{ \pm}<\min \left(\frac{\sqrt{2} \pi}{\zeta_{p}}, \sqrt{2} \pi\right)=\frac{\sqrt{2} \pi}{\zeta_{p}}, \quad \zeta_{p}^{2} \equiv\left(\frac{c-1}{12 g}\right) .
$$

As a result, we deduce the universal inequality satisfied by the "areal" spectral gap $A$ and twist gap $g$ :

$$
A g \leq \frac{\pi(c-1) r^{2}}{12}
$$

where we have shown $r=\frac{4 \sqrt{3}}{\pi} \simeq 2.21>1$ and we suspect that it can be made to 1 .

\footnotetext{
${ }^{7}$ Should we not assume twist gap, we would have $\Lambda_{ \pm}<\frac{\sqrt{2} \pi}{\gamma}$, just like the analysis for all the operators without assuming twist gap.
} 


\section{Lemma: density of states on strip of order one width}

In this section, we prove a lemma which is going to play a pivotal role in the next section, where we are going to prove an asymptotic result for the integrated density of states i.e. number of states upto a large $(h, \bar{h})$ threshold. This also helps us to derive the asymptotic spectral gap via strip like regions as defined in 1.4.2. We start by defining the following functions

$$
\begin{aligned}
& Q(h, \bar{\beta}) \equiv \int_{h-\delta}^{h+\delta} \mathrm{d} h^{\prime} \int_{0}^{\infty} \mathrm{d} \bar{h}^{\prime} \rho\left(h^{\prime}, \bar{h}^{\prime}\right) e^{-\bar{\beta} \bar{h}^{\prime}}, \\
& P(\bar{h}, \beta) \equiv \int_{\bar{h}-\bar{\delta}}^{\bar{h}+\bar{\delta}} \mathrm{d} \bar{h}^{\prime} \int_{0}^{\infty} \mathrm{d} h^{\prime} \rho\left(h^{\prime}, \bar{h}^{\prime}\right) e^{-\beta h^{\prime}} .
\end{aligned}
$$

The aim of this section is to prove the following lemma:

$$
\begin{aligned}
& e^{\bar{\beta} \bar{h}} Q(h, \bar{\beta})_{\bar{\beta}=\pi \sqrt{\frac{c}{6 \bar{h}}}}^{=} O\left(h^{-3 / 4} \exp \left[2 \pi\left(\sqrt{\frac{c h}{6}}+\sqrt{\frac{c \bar{h}}{6}}\right)\right]\right), \\
& e^{\beta h} P(\bar{h}, \beta)_{\substack{\beta=\pi \sqrt{\frac{c}{6 h}} \\
=}}^{=} O\left(\bar{h}^{-3 / 4} \exp \left[2 \pi\left(\sqrt{\frac{c h}{6}}+\sqrt{\frac{c \bar{h}}{6}}\right)\right]\right) .
\end{aligned}
$$

Let us focus on the quantity $Q$, the argument for $P$ follows in a similar manner. In order to estimate $Q$, we write down the master inequality:

$$
\begin{aligned}
& e^{\beta(h-\delta)} \int_{0}^{\infty} d h^{\prime} \int_{0}^{\infty} \mathrm{d} \bar{h}^{\prime} \rho\left(h^{\prime}, \bar{h}^{\prime}\right) \phi_{-}\left(h^{\prime}\right) e^{-\beta h^{\prime}-\bar{\beta} \bar{h}^{\prime}} \\
& \leq Q(h, \bar{\beta}) \\
& \leq e^{\beta(h+\delta)} \int_{0}^{\infty} d h^{\prime} \int_{0}^{\infty} \mathrm{d} \bar{h}^{\prime} \rho\left(h^{\prime}, \bar{h}^{\prime}\right) \phi_{+}\left(h^{\prime}\right) e^{-\beta h^{\prime}-\bar{\beta} \bar{h}^{\prime}},
\end{aligned}
$$

where we have used

$$
\phi_{-}\left(h^{\prime}\right) \leq \Theta\left(h^{\prime} \in[h-\delta, h+\delta]\right) \leq \phi_{+}\left(h^{\prime}\right) .
$$

Next we note that

$$
\begin{aligned}
& \int_{0}^{\infty} \mathrm{d} h^{\prime} \int_{0}^{\infty} \mathrm{d} \bar{h}^{\prime} \rho\left(h^{\prime}, \bar{h}^{\prime}\right) \phi_{ \pm}\left(h^{\prime}\right) e^{-\beta h^{\prime}-\bar{\beta} \bar{h}^{\prime}} \\
& =e^{-\beta c / 24-\bar{\beta} c / 24} \int_{-\infty}^{\infty} \mathrm{d} t e^{-\imath t c / 24} Z(\beta+\imath t, \bar{\beta}) \hat{\phi}_{ \pm}(t) \\
& =e^{-\beta c / 24-\bar{\beta} c / 24} \int_{-\infty}^{\infty} \mathrm{d} t e^{-\imath t c / 24} Z\left(\frac{4 \pi^{2}}{\beta+\imath t}, \frac{4 \pi^{2}}{\bar{\beta}}\right) \hat{\phi}_{ \pm}(t) .
\end{aligned}
$$

Now we separate the contribution to $Z\left(\frac{4 \pi^{2}}{\beta+\imath t}, \frac{4 \pi^{2}}{\beta}\right)$ into two pieces $Z_{L}$ (contribution from the "light" sector) and $Z_{H}$ (contribution from the "heavy" sector). The inequality 
in (4.4) can be written as

$$
\begin{aligned}
& e^{\beta(h-c / 24-\delta)-\bar{\beta} c / 24} \int_{-\infty}^{\infty} \mathrm{d} t e^{-\imath t c / 24} Z_{L}\left(\frac{4 \pi^{2}}{\beta+\imath t}, \frac{4 \pi^{2}}{\bar{\beta}}\right) \hat{\phi}_{-}(t) \\
& -e^{\beta(h-c / 24-\delta)-\bar{\beta} c / 24}\left|\int_{-\infty}^{\infty} \mathrm{d} t e^{-\imath t c / 24} Z_{H}\left(\frac{4 \pi^{2}}{\beta+\imath t}, \frac{4 \pi^{2}}{\bar{\beta}}\right) \hat{\phi}_{-}(t)\right| \\
& \leq Q(h, \bar{\beta}) \\
& \leq e^{\beta(h-c / 24+\delta)-\bar{\beta} c / 24} \int_{-\infty}^{\infty} \mathrm{d} t e^{-\imath t c / 24} Z_{L}\left(\frac{4 \pi^{2}}{\beta+\imath t}, \frac{4 \pi^{2}}{\bar{\beta}}\right) \hat{\phi}_{+}(t) \\
& +e^{\beta(h-c / 24+\delta)-\bar{\beta} c / 24}\left|\int_{-\infty}^{\infty} \mathrm{d} t e^{-\imath t c / 24} Z_{H}\left(\frac{4 \pi^{2}}{\beta+\imath t}, \frac{4 \pi^{2}}{\bar{\beta}}\right) \hat{\phi}_{+}(t)\right| .
\end{aligned}
$$

For notational simplicity, let us name the terms

$$
\begin{aligned}
& I_{ \pm}^{1}=e^{\beta(h-c / 24 \pm \delta)-\bar{\beta} c / 24} \int_{-\infty}^{\infty} \mathrm{d} t e^{-\imath t c / 24} Z_{L}\left(\frac{4 \pi^{2}}{\beta+\imath t}, \frac{4 \pi^{2}}{\bar{\beta}}\right) \hat{\phi}_{ \pm}(t) \\
& I_{ \pm}^{2}=e^{\beta(h-c / 24 \pm \delta)-\bar{\beta} c / 24}\left|\int_{-\infty}^{\infty} \mathrm{d} t e^{-\imath t c / 24} Z_{H}\left(\frac{4 \pi^{2}}{\beta+\imath t}, \frac{4 \pi^{2}}{\bar{\beta}}\right) \hat{\phi}_{ \pm}(t)\right|
\end{aligned}
$$

The idea is to show that $I_{ \pm}^{2}$ is subleading with respect to $I_{ \pm}^{1}$. The argument closely follows the argument presented in section 3. Let us concentrate on $I_{ \pm}^{2}$ first. We have

$$
I_{ \pm}^{2} \leq e^{\beta(h-c / 24 \pm \delta)-\bar{\beta} c / 24} \int_{-\infty}^{\infty} \mathrm{d} t Z_{H}\left(\frac{4 \pi^{2} \beta}{\beta^{2}+t^{2}}, \frac{4 \pi^{2}}{\bar{\beta}}\right)\left|\hat{\phi}_{ \pm}(t)\right|
$$

We notice that (for the heavy sector, $h^{\prime}+\bar{h}^{\prime}>c / 12$, thus one of them has to be greater than $c / 24)$

$$
\begin{aligned}
& Z_{H}\left(\frac{4 \pi^{2} \beta}{\beta^{2}+t^{2}}, \frac{4 \pi^{2}}{\bar{\beta}}\right) \ni e^{-\frac{4 \pi^{2} \beta}{\beta^{2}+t^{2}}\left(h^{\prime}-\frac{c}{24}\right)-\frac{4 \pi^{2}}{\beta}\left(\bar{h}^{\prime}-\frac{c}{24}\right)} \\
& \leq \begin{cases}e^{\frac{\pi^{2} c}{6 \beta}} e^{-\frac{4 \pi^{2} \beta}{\beta^{2}+\Lambda_{ \pm}^{2}} h^{\prime}-\frac{4 \pi^{2} \bar{\beta}}{\bar{\beta}^{2}+\Lambda_{ \pm}^{2}}\left(\bar{h}^{\prime}-\frac{c}{24}\right)}, & \bar{h}^{\prime}>c / 24 \\
e^{\frac{\pi^{2} c}{6 \beta}} e^{-\frac{4 \pi^{2} \beta}{\beta^{2}+\Lambda_{ \pm}^{2}}\left(h^{\prime}-\frac{c}{24}\right)-\frac{4 \pi^{2} \bar{\beta}}{\bar{\beta}^{2}+\Lambda_{ \pm}^{2}} \bar{h}^{\prime}}, & h^{\prime}>c / 24\end{cases} \\
& \leq \begin{cases}e^{\frac{\pi^{2} c}{6 \beta}} e^{-\frac{4 \pi^{2} \beta_{*}}{\beta_{*}^{2}+\Lambda_{ \pm}^{2}} h^{\prime}-\frac{4 \pi^{2} \beta_{*}}{\beta_{*}^{2}+\Lambda_{ \pm}^{2}}\left(\bar{h}^{\prime}-\frac{c}{24}\right)}, & \bar{h}^{\prime}>c / 24 \\
e^{\frac{\pi^{2} c}{6 \beta}} e^{-\frac{4 \pi^{2} \beta_{*}}{\beta_{*}^{2}+\Lambda_{ \pm}^{2}} h^{\prime}-\frac{4 \pi^{2} \beta_{*}}{\beta_{*}^{2}+\Lambda_{ \pm}^{2}}\left(\bar{h}^{\prime}-\frac{c}{24}\right)}, & h^{\prime}>c / 24\end{cases} \\
& \leq \begin{cases}e^{\frac{\pi^{2} c}{6 \beta}} e^{\frac{-\pi^{2} c \beta_{*}}{6\left(\beta_{*}^{2}+\Lambda_{ \pm}^{2}\right)}} e^{-\frac{4 \pi^{2} \beta_{*}}{\beta_{*}^{2}+\Lambda_{ \pm}^{2}}\left(h^{\prime}+\bar{h}^{\prime}-\frac{c}{12}\right)}, & \bar{h}^{\prime}>c / 24 \\
e^{\frac{\pi^{2} c}{6 \beta}} e^{-\frac{\pi^{2} c \beta_{*}}{6\left(\beta_{*}^{2}+\Lambda_{ \pm}^{2}\right)}} e^{-\frac{4 \pi^{2} \beta_{*}}{\beta_{*}^{2}+\Lambda_{ \pm}^{2}}\left(h^{\prime}+\bar{h}^{\prime}-\frac{c}{12}\right)}, & h^{\prime}>c / 24 .\end{cases}
\end{aligned}
$$


Thus we have

$$
\begin{aligned}
I_{ \pm}^{2} \leq & e^{\beta(h+c / 24 \pm \delta)-\bar{\beta} c / 24} \int_{-\infty}^{\infty} \mathrm{d} t Z_{H}\left(\frac{4 \pi^{2} \beta}{\beta^{2}+t^{2}}, \frac{4 \pi^{2}}{\bar{\beta}}\right)\left|\hat{\phi}_{ \pm}(t)\right| \\
& \leq \underset{\bar{\beta}, \beta<2 \pi}{\leq} e^{\beta(h-c / 24 \pm \delta)-\bar{\beta} c / 24}\left(e^{\frac{\pi^{2} c}{6 \beta}}+e^{\frac{\pi^{2} c}{6 \beta}}\right) e^{-\frac{\pi^{2} \beta_{*} c}{6\left(\beta_{*}^{2}+\Lambda_{ \pm}^{2}\right)}} Z_{H, \Delta}\left(\frac{4 \pi^{2} \beta_{*}}{\beta_{*}^{2}+\Lambda_{ \pm}^{2}}\right) \int_{-\infty}^{\infty} \mathrm{d} t\left|\hat{\phi}_{ \pm}(t)\right|
\end{aligned}
$$

The above analysis is analogous to the one presented in section 3. Now we choose

$$
\beta=\pi \sqrt{\frac{c}{6 h}}, \bar{\beta}=\pi \sqrt{\frac{c}{6 \bar{h}}},
$$

and use the HKS bound [19] to estimate $Z_{H, \Delta}\left(\frac{4 \pi^{2} \beta_{*}}{\beta_{*}^{2}+\Lambda_{ \pm}^{2}}\right)$. This leads to the following inequality:

$$
I_{ \pm}^{2} \leq e^{\pi \sqrt{\frac{c h}{6}}}\left(e^{\pi \sqrt{\frac{c \bar{h}}{6}}}+e^{\pi \sqrt{\frac{c h}{6}}}\right) \int_{-\infty}^{\infty} \mathrm{d} t\left|\hat{\phi}_{ \pm}(t)\right| \begin{cases}e^{2 \pi \sqrt{\frac{c h}{6}} \frac{\Lambda_{ \pm}^{2}}{4 \pi^{2}}} & h \geq \bar{h} \\ e^{2 \pi \sqrt{\frac{c h}{6}} \frac{\Lambda_{ \pm}^{2}}{4 \pi^{2}}} & h<\bar{h}\end{cases}
$$

To estimate $I_{ \pm}^{1}$, we consider $\rho_{*}(h, \bar{h})$, the crossing kernel, defined as

$$
\exp \left[\frac{\pi^{2} c}{6 \beta}+\frac{\pi^{2} c}{6 \bar{\beta}}\right]=\int_{0}^{\infty} d h \int_{0}^{\infty} d \bar{h} \rho_{*}(h, \bar{h}) e^{-\beta(h-c / 24)-\bar{\beta}(\bar{h}-c / 24)} .
$$

Here $\rho_{*}(h, \bar{h})=\rho_{*}(h) \rho_{*}(\bar{h})$, and $\rho_{*}(h), \rho_{*}(\bar{h})$ are given by

$$
\begin{aligned}
\rho_{*}(x) & =\pi \sqrt{\frac{c}{6}} \frac{I_{1}\left(2 \pi \sqrt{\frac{c}{3}\left(x-\frac{c}{24}\right)}\right)}{\sqrt{x-\frac{c}{24}}} \theta\left(x-\frac{c}{24}\right)+\delta\left(x-\frac{c}{24}\right), \\
& =\underset{x \rightarrow \infty}{=}\left(\frac{c}{96 x^{3}}\right)^{\frac{1}{4}} \exp \left[2 \pi \sqrt{\frac{c x}{6}}\right] .
\end{aligned}
$$

Hence, we have

$$
\begin{aligned}
I_{ \pm}^{1} & =e^{\pi \sqrt{\frac{c h}{6}}} \int_{0}^{\infty} \mathrm{d} h^{\prime} \int_{0}^{\infty} \mathrm{d} \bar{h}^{\prime} \rho_{*}\left(h^{\prime}, \bar{h}^{\prime}\right) e^{-\sqrt{\frac{c}{6 h}} h^{\prime}-\sqrt{\frac{c}{6 h} \bar{h}^{\prime}}} \phi_{ \pm}\left(h^{\prime}\right) \\
& =2 \delta c_{ \pm} e^{\pi \sqrt{\frac{c \bar{h}}{6}}} \rho_{*}(h),
\end{aligned}
$$

where we have used separability of $\rho_{*}$ in the variable $h$ and $\bar{h}$ and have defined

$$
c_{ \pm}=\frac{1}{2} \int_{-\infty}^{\infty} d x \phi_{ \pm}(h+\delta x)
$$

Comparing the inequalities (4.17) and (4.14), we see that by choosing $\gamma \Lambda_{ \pm}<\sqrt{2} \pi$, with $\max (h, \bar{h})=\gamma^{4} \min (h, \bar{h})$; one can make $I_{ \pm}^{2}$ subleading, consequently in the $h, \bar{h} \rightarrow \infty$ limit we have

$$
2 \delta c_{-}\left(\frac{c}{96 \bar{h}^{3}}\right)^{-\frac{1}{4}} \rho_{*}(h, \bar{h}) \leq e^{\pi \sqrt{\frac{c \bar{h}}{6}}} Q(h) \leq 2 \delta c_{+}\left(\frac{c}{96 \bar{h}^{3}}\right)^{-\frac{1}{4}} \rho_{*}(h, \bar{h}) .
$$


By symmetry we obtain

$$
2 \delta c_{-}^{\prime}\left(\frac{c}{96 h^{3}}\right)^{-\frac{1}{4}} \rho_{*}(h, \bar{h}) \leq e^{\pi \sqrt{\frac{c h}{6}}} P(\bar{h}) \leq 2 \delta c_{+}^{\prime}\left(\frac{c}{96 h^{3}}\right)^{-\frac{1}{4}} \rho_{*}(h, \bar{h}) .
$$

The best possible value of $c_{ \pm}\left(c_{ \pm}^{\prime}\right)$ can be obtained from [3]. For the verification purpose, here we choose the function given in [2] for estimating $Q$

$$
\begin{aligned}
& \phi_{+}\left(h^{\prime}\right)=\left(\frac{\sin \left(\frac{\Lambda_{+} \delta}{4}\right)}{\frac{\Lambda_{+} \delta}{4}}\right)^{-4}\left(\frac{\sin \left(\frac{\Lambda_{+}\left(h-h^{\prime}\right)}{4}\right)}{\frac{\Lambda_{+}\left(h-h^{\prime}\right)}{4}}\right)^{4}, \\
& \phi_{-}\left(h^{\prime}\right)=\left(1-\left(\frac{h-h^{\prime}}{\delta}\right)^{2}\right)\left(\frac{\sin \left(\frac{\Lambda_{-}\left(h-h^{\prime}\right)}{4}\right)}{\frac{\Lambda_{-}\left(h-h^{\prime}\right)}{4}}\right)^{4} .
\end{aligned}
$$

The above function yields almost the same bound as found in [2], except for the fact that we have to take care of the constraint $\Lambda<\frac{\sqrt{2} \pi}{\gamma}$. In particular we find the following bounding function $s_{ \pm}(\delta)=\log c_{ \pm}$:

$$
\begin{aligned}
& c_{+}= \begin{cases}\frac{\pi}{3}\left(\frac{\pi \delta}{2 \sqrt{2} \gamma}\right)^{3}\left(\sin \left(\frac{\pi \delta}{2 \sqrt{2} \gamma}\right)\right)^{-4}, & \delta<\frac{\gamma a_{*}}{\sqrt{2} \pi} \\
2.02, & \delta \geq \frac{\gamma a_{*}}{\sqrt{2} \pi}\end{cases} \\
& c_{-}= \begin{cases}\frac{2 \sqrt{2} \gamma}{3 \pi \delta^{3}}\left(\delta^{2}-\frac{6 \gamma^{2}}{\pi^{2}}\right), & \delta<\frac{6 \gamma}{\sqrt{2} \pi} \\
0.46, & \delta \geq \frac{6 \gamma}{\sqrt{2} \pi}\end{cases}
\end{aligned}
$$

where $a_{*}=3.38$ [2]. We verify the eq. (4.19) in the section 6 using the above values of $c_{ \pm}$ (in particular, see the figure 11). Similar verification can be done for the eq. (4.20) as well.

The analysis with the assumption of twist gap $g$ proceeds as in the end of section 3 . We do not repeat the analysis here. We just state the result. In that scenario, one obtains

$$
\Lambda_{ \pm}<\min \left(\frac{\sqrt{2} \pi}{\zeta}, \sqrt{2} \pi\right), \quad \zeta^{2} \equiv\left(\frac{c}{12 g}\right) .
$$

If we make it specific for primaries, $c \mapsto c-1$ and we have

$$
\begin{aligned}
\Lambda_{ \pm} & <\min \left(\frac{\sqrt{2} \pi}{\zeta_{p}}, \sqrt{2} \pi\right) \\
& =\frac{\sqrt{2} \pi}{\zeta_{p}}, \quad \zeta_{p}^{2} \equiv\left(\frac{c-1}{12 g}\right) \geq 1 .
\end{aligned}
$$

where the second equality follows only if there is no conserved currents because of the bound on twist gap.

We can use this lemma to prove the result about asymptotic spectral gap in terms of strips, as mentioned in 1.4.2. For this purpose, we use the magic function introduced in [3]. We can let $\gamma=1$ in the above analysis. We have to keep in mind that now the support of the Fourier transform of $\phi_{-}$satisfies $\Lambda<\frac{\sqrt{2} \pi}{\gamma}$, thus the minimal value of $\delta$ comes out to be $\frac{1}{\sqrt{2}}$ in stead of $1 / 2$ as in [3]. 


\section{The integrated density of states}

\subsection{The main 2D Tauberian theorem}

We prove in this section

$$
\begin{aligned}
F(h, \bar{h}) & \equiv \int_{0}^{h} \mathrm{~d} h^{\prime} \int_{0}^{\bar{h}} \mathrm{~d} \bar{h}^{\prime} \rho\left(h^{\prime}, \bar{h}^{\prime}\right) \\
& =\frac{1}{h, \bar{h} \rightarrow \infty}\left(\frac{36}{4 \pi^{2}}\right)^{1 / 4} \exp \left[2 \pi\left(\sqrt{\frac{c h}{6}}+\sqrt{\frac{c \bar{h}}{6}}\right)\right]\left[1+O\left(\tau^{\frac{r}{4}-1 / 2}\right)\right] .
\end{aligned}
$$

where $\tau$ is the twist of the state with $h, \bar{h}$ and given by $\tau=2 \min \{h, \bar{h}\}$ and $h=\bar{h}^{v}$ with $1 / 2<v<2$ and $\Upsilon=\max (v, 1 / v)$. When $\Upsilon=1$, this reduces to the eq. (1.1). In order to prove this we define

$$
\begin{aligned}
\delta \rho(h, \bar{h}) & =\rho(h, \bar{h})-\rho_{*}(h, \bar{h}), \\
\delta \mathcal{L}(\beta, \bar{\beta}) & =\mathcal{L}_{\rho}(\beta, \bar{\beta})-\mathcal{L}_{\rho_{*}}(\beta, \bar{\beta}) .
\end{aligned}
$$

Since the leading term is already produced by $\rho_{*}(h, \bar{h})$, our job is to show that

$$
\begin{aligned}
& \int_{0}^{h} \mathrm{~d} h^{\prime} \int_{0}^{\bar{h}} \mathrm{~d} \bar{h}^{\prime} \delta \rho\left(h^{\prime}, \bar{h}^{\prime}\right) \\
& =\frac{1}{4 \pi^{2}}\left(\frac{36 c^{2}}{h \bar{h}}\right)^{1 / 4} \exp \left[2 \pi\left(\sqrt{\frac{c h}{6}}+\sqrt{\frac{c \bar{h}}{6}}\right)\right] O\left(\tau^{\Upsilon / 4-1 / 2}\right) .
\end{aligned}
$$

In particular, we will be showing that

$$
\begin{aligned}
& \int_{0}^{h} \mathrm{~d} h^{\prime} \int_{0}^{\bar{h}} \mathrm{~d} \bar{h}^{\prime} \delta \rho\left(h^{\prime}, \bar{h}^{\prime}\right) \\
& =\frac{\sqrt{6 c}}{4 \pi^{2}} \exp \left[2 \pi\left(\sqrt{\frac{c h}{6}}+\sqrt{\frac{c \bar{h}}{6}}\right)\right]\left[O\left(h^{-3 / 4}\right)+O\left(\bar{h}^{-3 / 4}\right)\right] .
\end{aligned}
$$

Thus if $v \in(1 / 2,2)$, the error term is suppressed by maximum of $\frac{h^{1 / 4}}{\sqrt{h}}$ and $\frac{\bar{h}^{1 / 4}}{\sqrt{h}}$, arriving at (5.3).

In order to prove the above, we proceed as in [2] and introduce the following kernel:

$$
\begin{array}{ll}
G(\nu)=\frac{1}{2 \pi \imath} \int_{\beta-\imath \Lambda}^{\beta+\imath \Lambda} \frac{d z}{z} \frac{\Lambda^{2}+(z-\beta)^{2}}{\Lambda^{2}+\beta^{2}} e^{-\nu z}, & \nu=h^{\prime}-h, \\
G(\bar{\nu})=\frac{1}{2 \pi \imath} \int_{\bar{\beta}-\imath \Lambda}^{\bar{\beta}+\imath \Lambda} \frac{d \bar{z}}{\bar{z}} \frac{\Lambda^{2}+(\bar{z}-\bar{\beta})^{2}}{\Lambda^{2}+\bar{\beta}^{2}} e^{-\bar{\nu} \bar{z}}, & \bar{\nu}=\bar{h}^{\prime}-\bar{h} .
\end{array}
$$

Here we have done slight abuse of notation. It is implicitly assumed that the function $G(\bar{\nu})$ depends on $\bar{\beta}$ instead of $\beta$. Now it can be shown that [2]:

$$
G(\nu) G(\bar{\nu})=\left[\theta(-\nu)+G_{+}(\nu) \theta(\nu)+G_{-}(\nu) \theta(-\nu)\right]\left[\theta(-\bar{\nu})+G_{+}(\bar{\nu}) \theta(\bar{\nu})+G_{-}(\bar{\nu}) \theta(-\bar{\nu})\right],
$$


where $G_{ \pm}$is defined exactly like in [2] for both the variable $\nu$ and $\bar{\nu}$. At this point, we use the kernel given in (5.7) and integrate it against $\delta \rho(\Delta)$. This yields us the following equation

$$
\begin{aligned}
& \int_{0}^{h} \mathrm{~d} h^{\prime} \int_{0}^{\bar{h}} \mathrm{~d} \bar{h}^{\prime} \delta \rho\left(h^{\prime}, \bar{h}^{\prime}\right)=\int_{0}^{\infty} \mathrm{d} h^{\prime} \int_{0}^{\infty} \mathrm{d} \bar{h}^{\prime} \delta \rho\left(h^{\prime}, \bar{h}^{\prime}\right) G(\nu) G(\bar{\nu}) \\
& +\int_{0}^{\infty} \mathrm{d} h^{\prime} \int_{0}^{\infty} \mathrm{d} \bar{h}^{\prime} \delta \rho\left(h^{\prime}, \bar{h}^{\prime}\right)\left[-\theta(-\bar{\nu}) \theta(\nu) G_{+}(\nu)-\theta(-\bar{\nu}) \theta(-\nu) G_{-}(\nu)-(\nu \rightarrow \bar{\nu})\right] \\
& +\int_{0}^{\infty} \mathrm{d} h^{\prime} \int_{0}^{\infty} \mathrm{d} \bar{h}^{\prime} \delta \rho\left(h^{\prime}, \bar{h}^{\prime}\right)\left[-\theta(-\bar{\nu}) G_{-}(\bar{\nu}) \theta(\nu) G_{+}(\nu)-\theta(-\bar{\nu}) G_{-}(\bar{\nu}) \theta(-\nu) G_{-}(\nu)\right] \\
& +\int_{0}^{\infty} \mathrm{d} h^{\prime} \int_{0}^{\infty} \mathrm{d} \bar{h}^{\prime} \delta \rho\left(h^{\prime}, \bar{h}^{\prime}\right)\left[-\theta(\bar{\nu}) G_{+}(\bar{\nu}) \theta(\nu) G_{+}(\nu)-\theta(\bar{\nu}) G_{+}(\bar{\nu}) \theta(-\nu) G_{-}(\nu)\right] .
\end{aligned}
$$

Most of the terms can be estimated using techniques from [2]. The new players in the game are the cross terms, for example the term:

$$
Z=\int_{0}^{h} \mathrm{~d} h^{\prime} \int_{0}^{\bar{h}} \mathrm{~d} \bar{h}^{\prime} \delta \rho\left(h^{\prime}, \bar{h}^{\prime}\right)\left[\theta(-\bar{\nu}) \theta(\nu) G_{+}(\nu)+\theta(-\bar{\nu}) \theta(-\nu) G_{-}(\nu)+(\nu \rightarrow \bar{\nu})\right] .
$$

Below we will illustrate how to handle these terms. We remark that this is what requires us to prove the lemma in the previous section. For concreteness, consider the following term $\theta(-\bar{\nu}) \theta(\nu) G_{+}(\nu)$ and analyze it carefully. The analysis for the other terms in $Z$ goes exactly in the same manner. In what follows, we will be using the inequalities for $\beta>0$ :

$$
\begin{aligned}
\theta(-\bar{\nu}) & \leq e^{-\bar{\beta} \bar{\nu}} \\
\left|G_{ \pm}(\nu)\right| & \leq 2 e^{-\beta \nu} \min \left(1,\left(h-h^{\prime}\right)^{-2}\right), \\
\left|G_{ \pm}(\bar{\nu})\right| & \leq 2 e^{-\bar{\beta} \bar{\nu}} \min \left(1,\left(\bar{h}-\bar{h}^{\prime}\right)^{-2}\right) .
\end{aligned}
$$

The inequalities (5.13) and (5.14) have been derived in the appendix of [2].

Consider the term

$$
Z 1=\int_{0}^{\infty} \mathrm{d} h^{\prime} \int_{0}^{\infty} \mathrm{d} \bar{h}^{\prime} \theta(-\bar{\nu}) \Theta(\nu) G(\nu) \delta \rho\left(h^{\prime}, \bar{h}^{\prime}\right) .
$$

Clearly, we have

$$
|Z 1| \leq 2 e^{\beta h+\bar{\beta} \bar{h}} \int_{0}^{\infty} \mathrm{d} h^{\prime} \int_{0}^{\infty} \mathrm{d} \bar{h}^{\prime} e^{-\beta h^{\prime}-\bar{\beta} \bar{h}^{\prime}}\left[\rho\left(h^{\prime}, \bar{h}^{\prime}\right)+\rho_{*}\left(h^{\prime}, \bar{h}^{\prime}\right)\right] \min \left(1,\left(h-h^{\prime}\right)^{-2}\right) .
$$

For the term with $\rho_{*}\left(h^{\prime}, \bar{h}^{\prime}\right)$, the estimation procedure mimics the one presented in the section 5 of [2]. In particular, we have

$$
\begin{aligned}
& e^{\beta h+\bar{\beta} \bar{h}} \int_{0}^{\infty} \mathrm{d} h^{\prime} \int_{0}^{\infty} \mathrm{d} \bar{h}^{\prime} e^{-\beta h^{\prime}-\bar{\beta} \bar{h}^{\prime}} \rho_{*}\left(h^{\prime}, \bar{h}^{\prime}\right) \min \left(1,\left(h-h^{\prime}\right)^{-2}\right) \\
& =O\left(h^{-3 / 4} e^{2 \pi\left(\sqrt{\frac{c h}{6}}+\sqrt{\frac{c \bar{h}}{6}}\right)}\right) .
\end{aligned}
$$


The $h^{\prime}$ integral is done via saddle point method, note it is important to have the factor $\min \left(1,\left(h-h^{\prime}\right)^{-2}\right)$ for the validity of saddle point approximation. This is why, the $\bar{h}^{\prime}$ integral can not be done using saddle, hence does not produce any polynomial suppression in $\bar{h}$. Now consider the term

$$
2 e^{\beta h+\bar{\beta} \bar{h}} \int_{0}^{\infty} \mathrm{d} h^{\prime} \int_{0}^{\infty} \mathrm{d} \bar{h}^{\prime} e^{-\beta h^{\prime}-\bar{\beta} \bar{h}^{\prime}} \rho\left(h^{\prime}, \bar{h}^{\prime}\right) \min \left(1,\left(h-h^{\prime}\right)^{2}\right),
$$

and we divide it into three pieces

$$
\begin{aligned}
& a_{1}=2 e^{\beta h+\bar{\beta} \bar{h}} \int_{0}^{h-h^{3 / 8}} \mathrm{~d} h^{\prime} \int_{0}^{\infty} \mathrm{d} \bar{h}^{\prime} e^{-\beta h^{\prime}-\bar{\beta} \bar{h}^{\prime}} \rho\left(h^{\prime}, \bar{h}^{\prime}\right) \min \left(1,\left(h-h^{\prime}\right)^{-2}\right), \\
& a_{2}=2 e^{\beta h+\bar{\beta} \bar{h}} \int_{h-h^{3 / 8}}^{h+h^{3 / 8}} \mathrm{~d} h^{\prime} \int_{0}^{\infty} \mathrm{d} \bar{h}^{\prime} e^{-\beta h^{\prime}-\bar{\beta} \bar{h}^{\prime}} \rho\left(h^{\prime}, \bar{h}^{\prime}\right) \min \left(1,\left(h-h^{\prime}\right)^{-2}\right) \\
& a_{3}=2 e^{\beta h+\bar{\beta} \bar{h}} \int_{h+h^{3 / 8}}^{\infty} \mathrm{d} h^{\prime} \int_{0}^{\infty} \mathrm{d} \bar{h}^{\prime} e^{-\beta h^{\prime}-\bar{\beta} \bar{h}^{\prime}} \rho\left(h^{\prime}, \bar{h}^{\prime}\right) \min \left(1,\left(h-h^{\prime}\right)^{-2}\right)
\end{aligned}
$$

The estimate for $a_{1}$ and $a_{3}$ again proceeds like in [2] and we obtain

$$
\begin{aligned}
& a_{1}=O\left(h^{-3 / 4} e^{2 \pi\left(\sqrt{\frac{c h}{6}}+\sqrt{\frac{c \bar{h}}{6}}\right)}\right), \\
& a_{3}=O\left(h^{-3 / 4} e^{2 \pi\left(\sqrt{\frac{c h}{6}}+\sqrt{\frac{c \bar{h}}{6}}\right)}\right) .
\end{aligned}
$$

The estimation of the term $a_{2}$ would require the lemma from the previous section 4 . We subdivide $a_{2}$ into three different parts:

$$
\begin{aligned}
& a_{21}=2 e^{\beta h+\bar{\beta} \bar{h}} \int_{h-h^{3 / 8}}^{h-1} \mathrm{~d} h^{\prime} \int_{0}^{\infty} \mathrm{d} \bar{h}^{\prime} e^{-\beta h^{\prime}-\bar{\beta} \bar{h}^{\prime}} \rho\left(h^{\prime}, \bar{h}^{\prime}\right) \min \left(1,\left(h-h^{\prime}\right)^{-2}\right), \\
& a_{22}=2 e^{\beta h+\bar{\beta} \bar{h}} \int_{h-1}^{h+1} \mathrm{~d} h^{\prime} \int_{0}^{\infty} \mathrm{d} \bar{h}^{\prime} e^{-\beta h^{\prime}-\bar{\beta} \bar{h}^{\prime}} \rho\left(h^{\prime}, \bar{h}^{\prime}\right) \min \left(1,\left(h-h^{\prime}\right)^{-2}\right), \\
& a_{23}=2 e^{\beta h+\bar{\beta} \bar{h}} \int_{h+1}^{h+h^{3 / 8}} \mathrm{~d} h^{\prime} \int_{0}^{\infty} \mathrm{d} \bar{h}^{\prime} e^{-\beta h^{\prime}-\bar{\beta} \bar{h}^{\prime}} \rho\left(h^{\prime}, \bar{h}^{\prime}\right) \min \left(1,\left(h-h^{\prime}\right)^{-2}\right) .
\end{aligned}
$$

We have already estimated $a_{22}$ in the previous section 4 . This is basically order one window. Since, the estimation of $a_{21}$ and $a_{23}$ proceeds in similar manner, we would demonstrate the estimation of the term $a_{21}$.

$$
\begin{aligned}
a_{21} & =2 e^{\beta h+\bar{\beta} \bar{h}} \int_{h-h^{3 / 8}}^{h-1} \mathrm{~d} h^{\prime} \int_{0}^{\infty} \mathrm{d} \bar{h}^{\prime} e^{-\beta h^{\prime}-\bar{\beta} \bar{h}^{\prime}} \rho\left(h^{\prime}, \bar{h}^{\prime}\right) \min \left(1,\left(h-h^{\prime}\right)^{-2}\right) \\
& =2 e^{\bar{\beta} \bar{h}} \sum_{k=2}^{h^{3 / 8}} \int_{h-k}^{h-k+1} \mathrm{~d} h^{\prime} \int_{0}^{\infty} \mathrm{d} \bar{h}^{\prime} e^{\beta\left(h-h^{\prime}\right)-\bar{\beta} \bar{h}^{\prime}} \rho\left(h^{\prime}, \bar{h}^{\prime}\right)\left(h-h^{\prime}\right)^{-2} \\
& \leq 2 e^{\bar{\beta} \bar{h}} \sum_{k=2}^{h^{3 / 8}} \frac{e^{\beta k}}{(k-1)^{2}} \int_{h-k}^{h-k+1} \mathrm{~d} h^{\prime} \int_{0}^{\infty} \mathrm{d} \bar{h}^{\prime} e^{-\bar{\beta} \bar{h}^{\prime}} \rho\left(h^{\prime}, \bar{h}^{\prime}\right)
\end{aligned}
$$




$$
\begin{aligned}
& =O\left(h^{-3 / 4} e^{2 \pi\left(\sqrt{\frac{c h}{6}}+\sqrt{\frac{c \bar{h}}{6}}\right)} \sum_{k=2}^{h^{3 / 8}} \frac{e^{\beta k}}{(k-1)^{2}}\right) \\
& =O\left(h^{-3 / 4} e^{2 \pi\left(\sqrt{\frac{c h}{6}}+\sqrt{\frac{c \bar{h}}{6}}\right)}\right),
\end{aligned}
$$

where going from the third line to the fourth line requires use of lemma proven in the previous section 4 .

The estimation of the terms in (5.9) and (5.10) requires us to divide the $(h, \bar{h})$ plane into 9 regions (see figure 7):

$$
\begin{aligned}
& R_{1}=\left\{\left(h^{\prime}, \bar{h}^{\prime}\right): h^{\prime} \in\left[0, h-h^{3 / 8}\right], \bar{h}^{\prime} \in\left[0, \bar{h}-\bar{h}^{3 / 8}\right]\right\}, \\
& R_{2}=\left\{\left(h^{\prime}, \bar{h}^{\prime}\right): h^{\prime} \in\left[0, h-h^{3 / 8}\right], \bar{h}^{\prime} \in\left[\bar{h}-\bar{h}^{3 / 8}, \bar{h}+\bar{h}^{3 / 8}\right]\right\}, \\
& R_{3}=\left\{\left(h^{\prime}, \bar{h}^{\prime}\right): h^{\prime} \in\left[0, h-h^{3 / 8}\right], \bar{h}^{\prime} \in\left[\bar{h}+\bar{h}^{3 / 8}, \infty\right]\right\}, \\
& R_{4}=\left\{\left(h^{\prime}, \bar{h}^{\prime}\right): h^{\prime} \in\left[h-h^{3 / 8}, h+h^{3 / 8}\right], \bar{h}^{\prime} \in\left[0, \bar{h}-\bar{h}^{3 / 8}\right]\right\}, \\
& R_{5}=\left\{\left(h^{\prime}, \bar{h}^{\prime}\right): h^{\prime} \in\left[h-h^{3 / 8}, h+h^{3 / 8}\right], \bar{h}^{\prime} \in\left[\bar{h}-\bar{h}^{3 / 8}, \bar{h}+\bar{h}^{3 / 8}\right]\right\}, \\
& R_{6}=\left\{\left(h^{\prime}, \bar{h}^{\prime}\right): h^{\prime} \in\left[h-h^{3 / 8}, h+h^{3 / 8}\right], \bar{h}^{\prime} \in\left[\bar{h}+\bar{h}^{3 / 8}, \infty\right]\right\}, \\
& R_{7}=\left\{\left(h^{\prime}, \bar{h}^{\prime}\right): h^{\prime} \in\left[h+h^{3 / 8}, \infty\right], \bar{h}^{\prime} \in\left[0, \bar{h}-\bar{h}^{3 / 8}\right]\right\}, \\
& R_{8}=\left\{\left(h^{\prime}, \bar{h}^{\prime}\right): h^{\prime} \in\left[h+h^{3 / 8}, \infty\right], \bar{h}^{\prime} \in\left[\bar{h}-\bar{h}^{3 / 8}, \bar{h}+\bar{h}^{3 / 8}\right]\right\}, \\
& R_{9}=\left\{\left(h^{\prime}, \bar{h}^{\prime}\right): h^{\prime} \in\left[h+h^{3 / 8}, \infty\right], \bar{h}^{\prime} \in\left[\bar{h}+\bar{h}^{3 / 8}, \infty\right]\right\} .
\end{aligned}
$$

Basically, we have to estimate $\sum_{i} S_{i}$ where $S_{i}$ is given by

$$
\begin{aligned}
S_{i} & =\int_{R_{i}} \mathrm{~d} h^{\prime} \mathrm{d} \bar{h}^{\prime}\left|\delta \rho\left(h^{\prime}, \bar{h}^{\prime}\right)\right|\left[\left|G_{+}(\nu) G_{+}(\bar{\nu})\right|+\left|G_{-}(\bar{\nu}) G_{+}(\nu)\right|+(+\leftrightarrow-)\right] \\
& \leq 4\left(S_{i}^{(1)}+S_{i}^{(2)}\right)
\end{aligned}
$$

where we have used $|\delta \rho| \leq \rho+\rho_{*}, \mid G_{ \pm}(\nu) \leq 2 e^{-\beta \nu} \min \left(1,\left(h-h^{\prime}\right)^{-2}\right)$, and $\mid G_{ \pm}(\bar{\nu}) \leq$ $2 e^{-\bar{\beta} \bar{\nu}} \min \left(1,\left(\bar{h}-\bar{h}^{\prime}\right)^{-2}\right)$. The appearance of 4 is due to the fact that there are 4 terms in the integrand defining $S_{i}$ and each is suppressed in a same manner. Here $S_{i}^{(1)}$ and $S_{i}^{(2)}$ are defined as

$$
\begin{aligned}
& S_{i}^{(1)}=4 \int_{R_{i}} \mathrm{~d} h^{\prime} \mathrm{d} \bar{h}^{\prime} \rho\left(h^{\prime}, \bar{h}^{\prime}\right) e^{-\beta \nu-\bar{\beta} \bar{\nu}} \min \left(1,\left(h-h^{\prime}\right)^{-2}\right) \min \left(1,\left(\bar{h}-\bar{h}^{\prime}\right)^{-2}\right), \\
& S_{i}^{(2)}=4 \int_{R_{i}} \mathrm{~d} h^{\prime} \mathrm{d} \bar{h}^{\prime} \rho_{*}\left(h^{\prime}, \bar{h}^{\prime}\right) e^{-\beta \nu-\bar{\beta} \bar{\nu}} \min \left(1,\left(h-h^{\prime}\right)^{-2}\right) \min \left(1,\left(\bar{h}-\bar{h}^{\prime}\right)^{-2}\right) .
\end{aligned}
$$




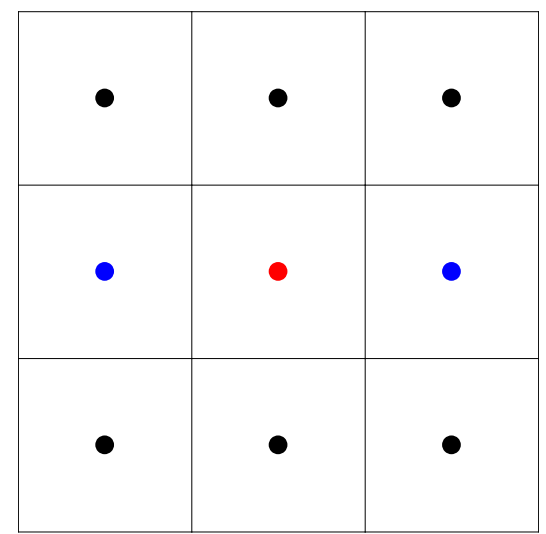

Figure 7. The estimation of the terms in (5.9) and (5.10) requires us to divide the $(h, \bar{h})$ plane into 9 regions. In the figure, the horizontal lines are $h^{\prime}=0, h-h^{3 / 8}, h+h^{3 / 8}, \infty$ while the vertical lines are $\bar{h}^{\prime}=0, \bar{h}-\bar{h}^{3 / 8}, \bar{h}+\bar{h}^{3 / 8}, \infty$. The different colors denote the different methods of treating them. As we will see, the region with red dot requires us to further subdivide it. We remark that the corner black ones can be colored blue as well, nonetheless in the main text, we have done the estimation in the "blue" way.

Now for $R_{i}$ with $i \neq 4,5,6$ we have

$$
\begin{aligned}
\frac{1}{4} S_{i \neq 4,5,6}^{(1)} & =e^{\beta h+\bar{\beta} \bar{h}} \int_{R_{i \neq 4,5,6}} \mathrm{~d} h^{\prime} \mathrm{d} \bar{h}^{\prime} \rho\left(h^{\prime}, \bar{h}^{\prime}\right) e^{-\beta h^{\prime}-\bar{\beta} \bar{h}^{\prime}} \min \left(1,\left(h-h^{\prime}\right)^{-2}\right) \min \left(1,\left(\bar{h}-\bar{h}^{\prime}\right)^{-2}\right) \\
& \leq e^{\beta h+\bar{\beta} \bar{h}} \int_{R_{i \neq 4,5,6}} \mathrm{~d} h^{\prime} \mathrm{d} \bar{h}^{\prime} \rho\left(h^{\prime}, \bar{h}^{\prime}\right) e^{-\beta h^{\prime}-\bar{\beta} \bar{h}^{\prime}} \min \left(1,\left(\bar{h}-\bar{h}^{\prime}\right)^{-2}\right) \\
& =O\left(\bar{h}^{-3 / 4} \exp \left[2 \pi\left(\sqrt{\frac{c h}{6}}+\sqrt{\frac{c \bar{h}}{6}}\right)\right]\right)
\end{aligned}
$$

For $R_{4}$ and $R_{6}$ we observe the following

$$
\begin{aligned}
\frac{1}{4} S_{i=4,6}^{(1)} & =e^{\beta h+\bar{\beta} \bar{h}} \int_{R_{i=4,6}} \mathrm{~d} h^{\prime} \mathrm{d} \bar{h}^{\prime} \rho\left(h^{\prime}, \bar{h}^{\prime}\right) e^{-\beta h^{\prime}-\bar{\beta} \bar{h}^{\prime}} \min \left(1,\left(h-h^{\prime}\right)^{-2}\right) \min \left(1,\left(\bar{h}-\bar{h}^{\prime}\right)^{-2}\right) \\
& \leq e^{\beta h+\bar{\beta} \bar{h}} \int_{R_{i=4,6}} \mathrm{~d} h^{\prime} \mathrm{d} \bar{h}^{\prime} \rho\left(h^{\prime}, \bar{h}^{\prime}\right) e^{-\beta h^{\prime}-\bar{\beta} \bar{h}^{\prime}} \min \left(1,\left(h-h^{\prime}\right)^{-2}\right) \\
& \leq e^{\beta h+\bar{\beta} \bar{h}} \bar{h}^{-3 / 4} \int_{R_{i=4,6}} \mathrm{~d} h^{\prime} \mathrm{d} \bar{h}^{\prime} \rho\left(h^{\prime}, \bar{h}^{\prime}\right) e^{-\beta h^{\prime}-\bar{\beta} \bar{h}^{\prime}} \\
& =O\left(h^{-3 / 4} \exp \left[2 \pi\left(\sqrt{\frac{c h}{6}}+\sqrt{\frac{c \bar{h}}{6}}\right)\right]\right) .
\end{aligned}
$$

For analyzing the region $R_{5}$, we are required to subdivide it into 9 regions again where each of the region is Cartesian product of order one interval in $h^{\prime}$ and $\bar{h}^{\prime}$. Now one can use 
the lemma proven in the section 3 to show that

$$
\begin{aligned}
\frac{1}{4} S_{5}^{(1)} & =e^{\beta h+\bar{\beta} \bar{h}} \int_{R_{5}} \mathrm{~d} h^{\prime} \mathrm{d} \bar{h}^{\prime} \rho\left(h^{\prime}, \bar{h}^{\prime}\right) e^{-\beta h^{\prime}-\bar{\beta} \bar{h}^{\prime}} \min \left(1,\left(h-h^{\prime}\right)^{-2}\right) \min \left(1,\left(\bar{h}-\bar{h}^{\prime}\right)^{-2}\right) \\
& =O\left(h^{-3 / 4} \bar{h}^{-3 / 4} \exp \left[2 \pi\left(\sqrt{\frac{c h}{6}}+\sqrt{\frac{c \bar{h}}{6}}\right)\right]\right) .
\end{aligned}
$$

The estimation for $S^{(2)}=\sum_{i} S_{i}^{(2)}$ can be done by saddle point method:

$$
\begin{aligned}
\frac{1}{4} S^{(2)} & =e^{\beta h+\bar{\beta} \bar{h}} \int_{0}^{\infty} \int_{0}^{\infty} \mathrm{d} h^{\prime} \mathrm{d} \bar{h}^{\prime} \rho_{*}\left(h^{\prime}, \bar{h}^{\prime}\right) e^{-\beta h^{\prime}-\bar{\beta} \bar{h}^{\prime}} \min \left(1, \frac{1}{\left(h-h^{\prime}\right)^{2}}\right) \min \left(1, \frac{1}{\left(\bar{h}-\bar{h}^{\prime}\right)^{2}}\right) \\
& =O\left(h^{-3 / 4} \bar{h}^{-3 / 4} \exp \left[2 \pi\left(\sqrt{\frac{c h}{6}}+\sqrt{\frac{c \bar{h}}{6}}\right)\right]\right) .
\end{aligned}
$$

We are yet to estimate the following term

$$
m \equiv \int_{0}^{\infty} \mathrm{d} h^{\prime} \int_{0}^{\infty} \mathrm{d} \bar{h}^{\prime} \delta \rho\left(h^{\prime}, \bar{h}^{\prime}\right) G(\nu) G(\bar{\nu}) .
$$

which appears in the expression for $\int_{0}^{h} \mathrm{~d} h^{\prime} \int_{0}^{\bar{h}} \mathrm{~d} \bar{h}^{\prime} \delta \rho\left(h^{\prime}, \bar{h}^{\prime}\right)$. Using the definition of $G(\nu)$ and $G(\bar{\nu})$, we arrive at

$$
m=-\frac{1}{4 \pi^{2}} \int_{\beta-\imath \Lambda}^{\beta+\imath \Lambda} \int_{\bar{\beta}-\imath \Lambda}^{\bar{\beta}+\imath \Lambda} \frac{d z d \bar{z}}{z \bar{z}} \frac{\Lambda^{2}+(z-\beta)^{2}}{\Lambda^{2}+\beta^{2}} \frac{\Lambda^{2}+(\bar{z}-\bar{\beta})^{2}}{\Lambda^{2}+\bar{\beta}^{2}} e^{z h+\bar{z} \bar{h}} \delta \mathcal{L}(z, \bar{z}) .
$$

Thus we have

$$
|m| \leq \frac{1}{4 \pi^{2}} \int_{-\Lambda}^{\Lambda} \int_{-\Lambda}^{\Lambda} \frac{\mathrm{d} t d \bar{t}}{|\beta+\imath t||\bar{\beta}+\imath \bar{t}|}\left(\frac{\Lambda^{2}-t^{2}}{\Lambda^{2}+\beta^{2}}\right)\left(\frac{\Lambda^{2}-\bar{t}^{2}}{\Lambda^{2}+\bar{\beta}^{2}}\right) e^{\beta h+\bar{\beta} \bar{h}}|\delta \mathcal{L}(z, \bar{z})| .
$$

Now we use the inequality

$$
|\delta \mathcal{L}(z, \bar{z})| \leq e^{-(\operatorname{Re}[z]+\operatorname{Re}[\bar{z}]) c / 24} Z_{H}\left(\frac{4 \pi^{2} \operatorname{Re}[z]}{|z|^{2}}, \frac{4 \pi^{2} \operatorname{Re}[\bar{z}]}{|\bar{z}|^{2}}\right),
$$

and subsequently the method utilized in section 3 to put a bound by $Z_{H, \Delta}$ :

$$
|m| \leq \frac{4 \Lambda^{6}}{9 \beta \bar{\beta} \pi^{2}} \frac{e^{\beta(h-c / 24)} e^{\bar{\beta}(\bar{h}-c / 24)} e^{\frac{\pi^{2} c}{6 y}}}{\left(\Lambda^{2}+\beta^{2}\right)\left(\Lambda^{2}+\bar{\beta}^{2}\right)} Z_{H, \Delta}\left(\frac{4 \pi^{2} \beta_{*}}{\beta_{*}^{2}+\Lambda^{2}}\right),
$$

where $y=\beta$ or $\bar{\beta}$. Essentially this is exactly the same argument as in section 3. Then by choosing $\Lambda<\sqrt{2} \pi$ (when $h \simeq \bar{h}$, otherwise we need to choose $\gamma \Lambda<\sqrt{2} \pi$, see the discussion in section 3) and using the HKS [19] argument, one can show that the above term is exponentially suppressed compared to the leading answer coming from $\rho_{*}(h, \bar{h})$. When $h$ is not of the order of $\bar{h}$, we need to assume existence of twist gap (as defined in 1.1) and proceed like we did in section 3. This concludes our analysis and hence the proof of the main theorem. 


\subsection{Sensitivity of asymptotics towards spin $J$}

The asymptotic formula given in eq. (1.1) and derived above can be rewritten in terms of dimension $\Delta=h+\bar{h}$ and $\operatorname{spin} J=|h-\bar{h}|$ :

$$
\begin{aligned}
& F(h \rightarrow \infty, \bar{h} \rightarrow \infty)=\int_{0}^{h} \mathrm{~d} h^{\prime} \int_{0}^{\bar{h}} \mathrm{~d} \bar{h}^{\prime} \rho\left(h^{\prime}, \bar{h}^{\prime}\right) \\
& =\frac{1}{4 \pi^{2}}\left(\frac{36}{c^{2}\left(\Delta^{2}-J^{2}\right)}\right)^{1 / 4} \exp \left[2 \pi\left(\sqrt{\frac{c(\Delta+J)}{12}}+\sqrt{\frac{c(\Delta-J)}{12}}\right)\right]\left[1+O\left(\Delta^{-1 / 4}\right)\right],
\end{aligned}
$$

which is true when $1<\frac{\Delta}{J}=O(1)$. It turns out that even when $\Delta$ and $J$ is not of the same order, we can do order by order correction to this integrated density of states by spin.

First of all, when $J$ is of order one, we should just ignore $J$ dependence of the eq. (5.40). In fact, $J=\Delta^{1 / n}$ with $n>4 / 3$, one can ignore $J$ dependence. Thus in this regime, we have

$$
\begin{aligned}
F\left(\Delta \rightarrow \infty, J=\Delta^{1 / n} \rightarrow \infty\right) & =\int_{0}^{h} \mathrm{~d} h^{\prime} \int_{0}^{\bar{h}} \mathrm{~d} \bar{h}^{\prime} \rho\left(h^{\prime}, \bar{h}^{\prime}\right), \quad 4 / 3<n \leq \infty \\
& =\frac{1}{4 \pi^{2}}\left(\frac{6}{c \Delta}\right)^{1 / 2} e^{2 \pi \sqrt{\frac{c \Delta}{3}}}\left[1+O\left(\Delta^{-1 / 4}\right)\right] .
\end{aligned}
$$

When $8 / 7 \leq n \leq 4 / 3$, the $J$ dependence is meaningful only within the exponential i.e. we have

$$
\begin{aligned}
F\left(\Delta \rightarrow \infty, J=\Delta^{1 / n} \rightarrow \infty\right) & =\int_{0}^{h} \mathrm{~d} h^{\prime} \int_{0}^{\bar{h}} \mathrm{~d} \bar{h}^{\prime} \rho\left(h^{\prime}, \bar{h}^{\prime}\right), \quad 8 / 7 \leq n \leq 4 / 3 \\
& =\frac{1}{4 \pi^{2}}\left(\frac{6}{c \Delta}\right)^{1 / 2} e^{2 \pi \sqrt{\frac{c \Delta}{3}}\left(1-\frac{J^{2}}{8 \Delta^{2}}\right)}\left[1+O\left(\Delta^{-1 / 4}\right)\right] .
\end{aligned}
$$

When $16 / 15<n<8 / 7$ ( $n$ can not less than one because of unitarity bound), we have

$$
\begin{aligned}
& F\left(\Delta \rightarrow \infty, J=\Delta^{1 / n} \rightarrow \infty\right)=\int_{0}^{h} \mathrm{~d} h^{\prime} \int_{0}^{\bar{h}} \mathrm{~d} \bar{h}^{\prime} \rho\left(h^{\prime}, \bar{h}^{\prime}\right), \quad 1<n<8 / 7 \\
& =\frac{1}{4 \pi^{2}}\left(\frac{6}{c \Delta}\right)^{1 / 2}\left(1+\frac{J^{2}}{4 \Delta^{2}}\right) \exp \left[2 \pi\left(\sqrt{\frac{c(\Delta+J)}{12}}+\sqrt{\frac{c(\Delta-J)}{12}}\right)\right]\left[1+O\left(\Delta^{-1 / 4}\right)\right] .
\end{aligned}
$$

We remark that not all the term in the exponential are meaningful, we have to do an $J / \Delta$ expansion and the only meaningful terms are of the form $\exp \left(\Delta^{-\ell}\right)$ with $\ell<1 / 4$ (since $\exp \left(\Delta^{-\ell}\right) \simeq 1+\Delta^{-\ell}$ becomes comparable to error term for $\left.\ell<1 / 4\right)$. For example, when $n=\frac{15}{14}$, it is meaningful to keep the following terms only:

$$
e^{2 \pi\left(\sqrt{\frac{c(\Delta+J)}{12}}+\sqrt{\frac{c(\Delta-J)}{12}}\right)}=e^{2 \pi \sqrt{\frac{c \Delta}{3}}\left(1-\frac{J^{2}}{8 \Delta^{2}}-\frac{5 J^{4}}{128 \Delta^{4}}-\frac{21 J^{6}}{1024 \Delta^{6}}-\frac{429 J^{8}}{32768 \Delta^{8}}-\frac{2431 J^{10}}{262144 \Delta^{10}}\right)}\left[1+O\left(\Delta^{-3 / 10}\right)\right] .
$$




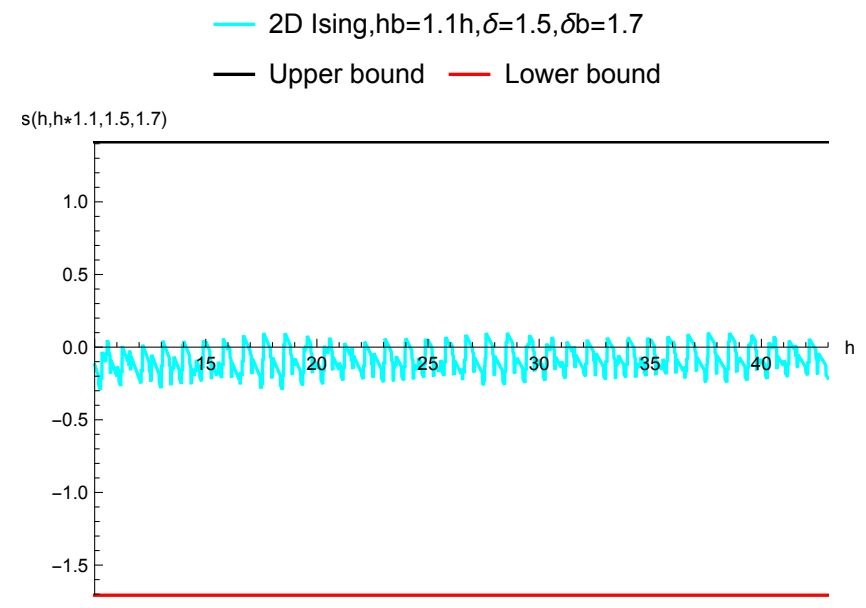

Figure 8. Verifying the bounds on $O(1)$ correction to entropy $(s(\delta, \bar{\delta}, h, \bar{h}))$ associated with order one window centered at some large $h$ and $\bar{h}$, the black line is the upper bound while the red line below is the lower bound. The curvy cyan line is the difference between the actual number of states lying within the window and leading answer coming from the Cardy formula. Here $h$ and $\bar{h}$ are of the same order.

One can generalize the equation (5.43) for $J=\Delta^{1 / n}$ with $\frac{2^{m+3}}{2^{m+3}-1}<n<\frac{2^{m+2}}{2^{m+2}-1} \leq 8 / 7$ where $m \geq 1$ is a fixed integer. We define $f(m)=\frac{2^{m}}{2^{m}-1}$ and we have:

$$
\begin{aligned}
& F\left(\Delta \rightarrow \infty, J=\Delta^{1 / n} \rightarrow \infty\right)=\int_{0}^{h} \mathrm{~d} h^{\prime} \int_{0}^{\bar{h}} \mathrm{~d} \bar{h}^{\prime} \rho\left(h^{\prime}, \bar{h}^{\prime}\right), \quad f(m+3)<n<f(m+2) \\
& =\frac{1}{4 \pi^{2}}\left(\frac{6}{c \Delta}\right)^{1 / 2}\left(\sum_{k=0}^{m} a_{k}\left(\frac{J^{2}}{\Delta^{2}}\right)^{k}\right) \exp \left[2 \pi\left(\sqrt{\frac{c(\Delta+J)}{12}}+\sqrt{\frac{c(\Delta-J)}{12}}\right)\right]\left[1+O\left(\Delta^{-1 / 4}\right)\right] .
\end{aligned}
$$

where $a_{k}$ 's are defined as

$$
\left(1-\frac{J^{2}}{\Delta^{2}}\right)^{1 / 4}=\sum_{k=0}^{\infty} a_{k}\left(\frac{J^{2}}{\Delta^{2}}\right)^{k}, \Delta>J
$$

\section{Verification: 2D Ising model}

We verify the bounds on $O(1)$ correction to entropy associated with order one window centered at some large $h$ and $\bar{h}$, proven in section 3 as shown in the figure 8, 9 and 10 . In section 3, we have obtained two kinds of bounds, one without assuming any twist gap while the other one assumes existence of a twist gap. For 2D Ising model, it turns out that the bound coming from assuming a twist gap (indeed 2D Ising model has a twist gap) is stronger than the one without using the information about twist gap. Thus in the figures below, we verify the bounds that uses the information about twist gap. In fact, as we have mentioned in section 3, the use of twist gap actually enables us to probe regions where $h$ and $\bar{h}$ are not of the same order. The figure 10 elucidates such a scenario. 


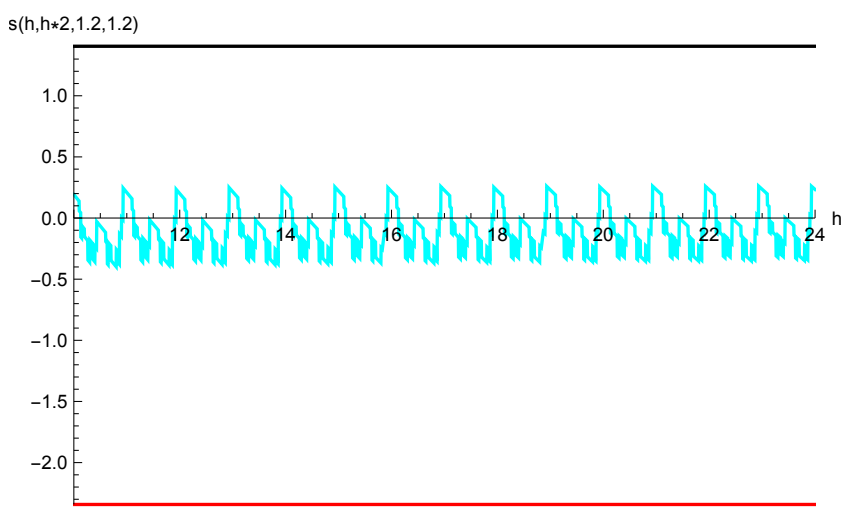

Figure 9. Verifying the bounds on $O(1)$ correction to entropy $(s(\delta, \bar{\delta}, h, \bar{h}))$ associated with order one window centered at some large $h$ and $\bar{h}$, the black line is the upper bound while the red line below is the lower bound. The curvy cyan line is the difference between the actual number of states lying within the window and leading answer coming from the Cardy formula. Here $h$ and $\bar{h}$ are of the same order.

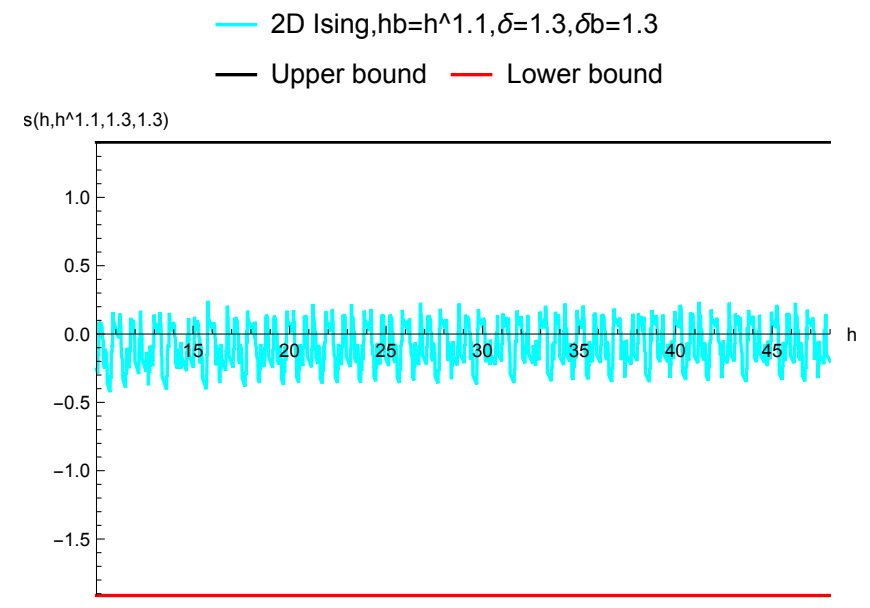

Figure 10. Verifying the bounds on $O(1)$ correction to entropy $(s(\delta, \bar{\delta}, h, \bar{h}))$ associated with order one window centered at some large $h$ and $\bar{h}$, the black line is the upper bound while the red line below is the lower bound. The curvy cyan line is the difference between the actual number of states lying within the window and leading answer coming from the Cardy formula. Here $h$ and $\bar{h}$ are not of the same order.

We verify the lemma proven in section 4 as shown in the figure 11 and figure 12 using 2 dimensional Ising CFT. The partition function for 2 dimensional Ising CFT is given by

$$
Z_{\text {Ising }}(\beta, \bar{\beta})=\frac{1}{2}\left(\sqrt{\frac{\theta_{2}(\beta)}{\eta(\beta)}} \sqrt{\frac{\theta_{2}(\bar{\beta})}{\eta(\bar{\beta})}}+\sqrt{\frac{\theta_{3}(\beta)}{\eta(\beta)}} \sqrt{\frac{\theta_{3}(\bar{\beta})}{\eta(\bar{\beta})}}+\sqrt{\frac{\theta_{4}(\beta)}{\eta(\beta)}} \sqrt{\frac{\theta_{4}(\bar{\beta})}{\eta(\bar{\beta})}}\right) .
$$

We verify the main theorem proven in section 5 in figure 13 and 14 . We plot the total number of states upto some $(h, \bar{h})$ as a function of $h$. We have considered two different 


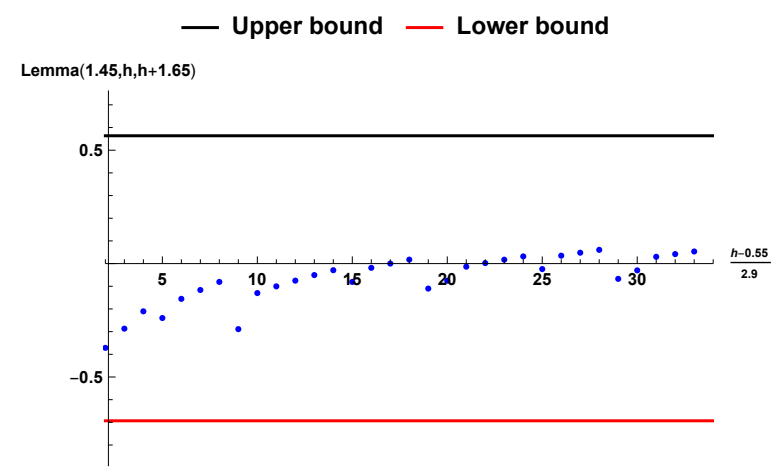

Figure 11. The blue dots denotes the values of $\operatorname{Lemma}(\delta, h, \bar{h}) \equiv \log \left[\frac{1}{2 \delta} Q\left(h, \bar{\beta}=\pi \sqrt{\frac{c \bar{h}}{6}}\right)\right]-$ $2 \pi \sqrt{\frac{c h}{6}}-\pi \sqrt{\frac{c \bar{h}}{6}}$, here we have $\delta=1.45$ and $\bar{h}=h+1.65$, for different values of $h$. The blue dots are bounded by an order one i.e. $h, \bar{h}$ independent number, denoted by the red and the black curve. The values of $c_{ \pm}$found in section 4 are used as the bounds.

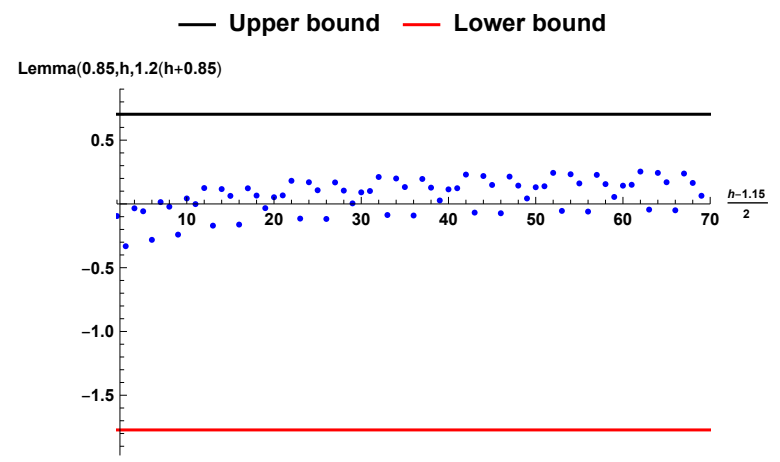

Figure 12. The blue dots denotes the values of $\operatorname{Lemma}(\delta, h, \bar{h}) \equiv \log \left[\frac{1}{2 \delta} Q\left(h, \bar{\beta}=\pi \sqrt{\frac{c \bar{h}}{6}}\right)\right]-$ $2 \pi \sqrt{\frac{c h}{6}}-\pi \sqrt{\frac{c \bar{h}}{6}}$, where $\delta=1.7$ and $\bar{h}=1.2(h+0.85)$, for different values of $h$. The blue dots are bounded by an order one i.e. $h, \bar{h}$ independent number, denoted by the red and the black curve. The $c_{ \pm}$found in the section 4 are used as the bounds.

cases where the asymptote is approached along different curves. We compare it against the asymptotic formula we derive in section 5 .

\section{$7 \quad$ Finite twist-large spin}

In this section, our aim is to estimate the finite twist and large spin sector of the density of states. Without loss of generality, we will assume $h$ is finite and $\bar{h} \rightarrow \infty$. We will probe the following quantity $U_{h}(\bar{h})$ in the limit $\bar{h} \rightarrow \infty$ :

$$
U_{h}(\bar{h})=\int_{0}^{h} \mathrm{~d} h^{\prime} \int_{\bar{h}-\bar{\delta}}^{\bar{h}+\bar{\delta}} \mathrm{d} \bar{h}^{\prime} \rho\left(h^{\prime}, \bar{h}^{\prime}\right) .
$$




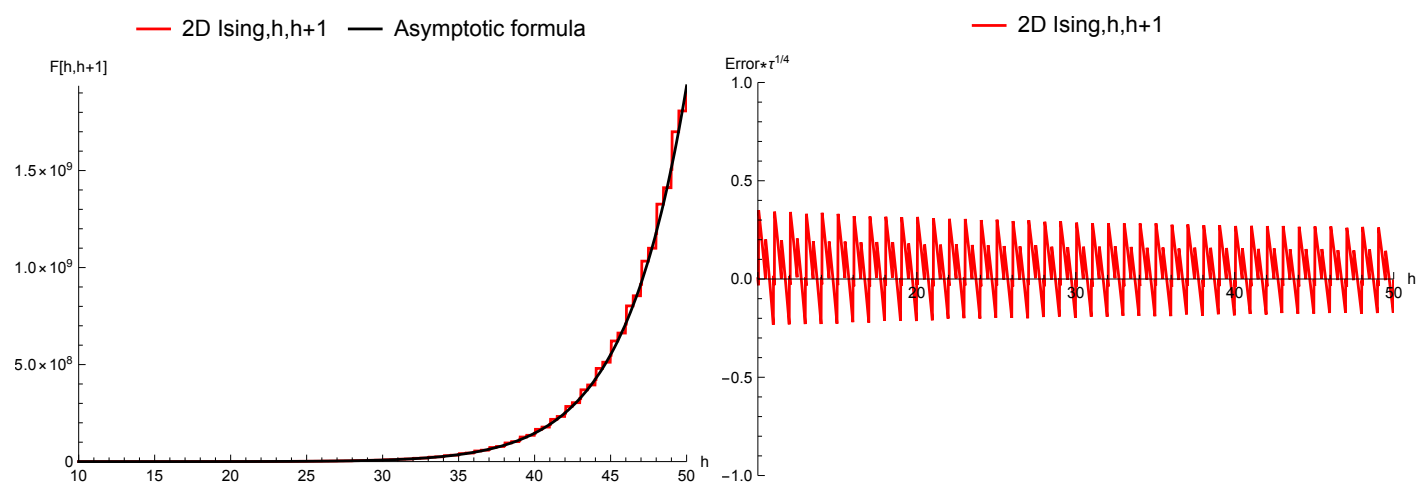

Figure 13. In the left figure, the black curve is the asymptotic Cardy formula while the red curve is the actual number of operators till $(h, \bar{h})$ present in the Ising model. Here we have taken $h=\bar{h}-1$. The picture on the right hand side plot the relative error times $h^{1 / 4}$. The error times $h^{1 / 4}$ is bounded above and below by an order one number.

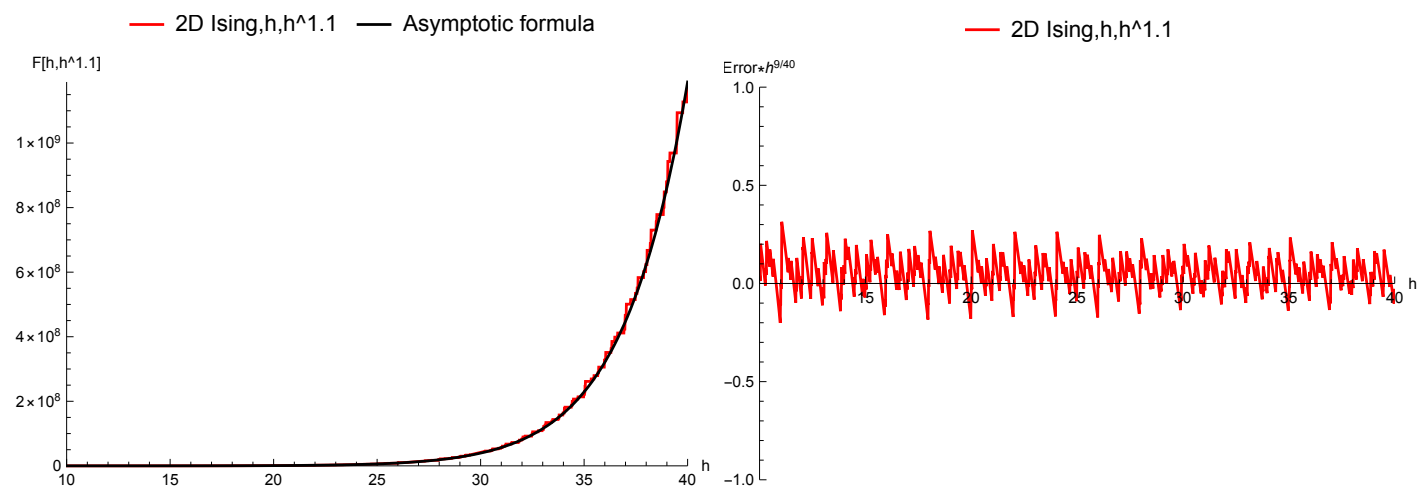

Figure 14. In the left figure, the black curve is the asymptotic Cardy formula while the red curve is the actual number of operators till $(h, \bar{h})$ present in the Ising model. Here we have taken $h^{1.1}=\bar{h}$. The picture on the right hand side plot the relative error times $h^{9 / 40}$. The error times $h^{9 / 40}$ is bounded above and below by an order one number.

This is because we have

$$
\int_{h-\delta}^{h+\delta} \mathrm{d} h^{\prime} \int_{\bar{h}-\bar{\delta}}^{\bar{h}+\bar{\delta}} \mathrm{d} \bar{h}^{\prime} \rho\left(h^{\prime}, \bar{h}^{\prime}\right) \leq U_{h+\delta}(\bar{h})
$$

Intuitively, it is clear that one can not make both $\beta$ and $\bar{\beta}$ to approach zero while looking at the partition function, as this would probe the regime $h, \bar{h} \rightarrow \infty$. This suggests that we should let $\bar{\beta} \rightarrow 0$ and keep $\beta$ fixed.

We can prove an upper bound on density of states in this sector. Let us write down the following inequality:

$$
U_{h}(\bar{h}) \leq e^{\beta(h-c / 24)} \int_{0}^{\infty} \mathrm{d} h^{\prime} \int_{\bar{h}-\bar{\delta}}^{\bar{h}+\bar{\delta}} \mathrm{d} \bar{h}^{\prime} \rho\left(h^{\prime}, \bar{h}^{\prime}\right) e^{-\beta\left(h^{\prime}-c / 24\right)}
$$


from which we can write

$$
U_{h}(\bar{h}) \leq e^{\beta\left(h-\frac{c}{24}\right)+\bar{\beta} \bar{h}+\bar{\beta} \bar{\delta}} \int_{0}^{\infty} \mathrm{d} h^{\prime} \int_{\bar{h}-\bar{\delta}}^{\bar{h}+\bar{\delta}} \mathrm{d} \bar{h}^{\prime} \rho\left(h^{\prime}, \bar{h}^{\prime}\right) \phi_{+}\left(\bar{h}^{\prime}\right) e^{-\beta\left(h^{\prime}-\frac{c}{24}\right)-\bar{\beta} \bar{h}^{\prime}} .
$$

At this point we choose

$$
\beta=2 \pi, \quad \bar{\beta}=\pi \sqrt{\frac{c}{6 \bar{h}}}, \bar{h} \rightarrow \infty .
$$

Again we separate the partition function into two pieces; the light sector, where the contribution comes from two kinds of states: a) all the states with conformal weight $\left(h^{\prime}, 0\right)$ with $h^{\prime} \geq 0$ and b) the states such that $h^{\prime}+\bar{h}^{\prime}<\frac{c}{12}$, and the heavy sector, which is defined to be the complement of this. We remark the heavy sector does not contain any operator with conformal weight $\left(h^{\prime}, 0\right)$. In the usual asymptotic analysis as done previously, the operators with $\left(h^{\prime}, 0\right)$ such that $h^{\prime}>\frac{c}{12}$ is put into the heavy sector, but here we can not do that since $\beta$ is finite, there is no separation between light or heavy in the sense of HKS [19] i.e. the operators with $\left(h^{\prime}, 0\right)$ contribute on equal footing as the operator $(0,0)$. The upshot of the above discussion is that one can define a kernel $\rho_{*}^{\mathrm{ft}}$ ("ft" stands for finite twist) such that

$$
\int_{0}^{\infty} \mathrm{d} h^{\prime} \int_{0}^{\infty} \mathrm{d} \bar{h}^{\prime} \rho_{*}^{\mathrm{ft}}\left(h^{\prime}, \bar{h}^{\prime}\right) e^{-\beta\left(h^{\prime}-\frac{c}{24}\right)-\bar{\beta} \bar{h}^{\prime}}=e^{\frac{\pi^{2} c}{6 \bar{\beta}}} \sum_{h_{i}} e^{-\beta\left(h_{i}-\frac{c}{24}\right)},
$$

where the sum on the right hand side is over all the states with weight $\left(h_{i}, 0\right)$ and we have used $\beta=\frac{4 \pi^{2}}{\beta}=2 \pi$. As a result $\rho_{*}^{\mathrm{ft}}$ is given by

$$
\rho_{*}^{\mathrm{ft}}\left(h^{\prime}, \bar{h}^{\prime}\right)=\rho_{*}\left(\bar{h}^{\prime}\right) \sum_{h_{i}} \delta\left(h^{\prime}-h_{i}\right)
$$

Once we have defined $\rho_{*}$, we follow the usual method and our next aim is to show that the heavy part has a subleading contribution. The estimation of the heavy part is done depending on whether both $h^{\prime}$ and $\bar{h}^{\prime}$ is greater than $c / 24$ or one of them is less than $c / 24$. In the later case, we have to assume existence of twist gap. Last but not the least, we have to estimate the contribution from the states with $\left(0, \bar{h}^{\prime}\right)$ with $\bar{h}^{\prime}>c / 12$. Following the methods in section 3 , we can show that they are indeed subleading. The leading answer is then given by

$$
U_{h}(\bar{h}) \leq e^{2 \pi\left(h-\frac{c}{24}\right)+\pi \sqrt{\frac{c \bar{h}}{6}}} \int \mathrm{d} h^{\prime} \int \mathrm{d} \bar{h}^{\prime} \rho_{*}^{\mathrm{ft}}\left(h^{\prime}, \bar{h}^{\prime}\right) e^{-2 \pi\left(h^{\prime}-\frac{c}{24}\right)-\pi \sqrt{\frac{c}{6 h} \bar{h}^{\prime}}} \Phi_{+}\left(\bar{h}^{\prime}\right) .
$$

The $\bar{h}^{\prime}$ integral can be done using saddle point approximation and we obtain

$$
\begin{aligned}
& \frac{1}{2 \bar{\delta}} U_{h}(\bar{h}) \leq e^{-\frac{\pi c}{12}} e^{2 \pi h} c_{+} \rho_{*}\left(\bar{h}^{\prime}\right) \sum_{h_{i}} e^{-2 \pi\left(h_{i}-\frac{c}{24}\right)}, \\
& \frac{1}{2 \bar{\delta}} U_{h}(\bar{h}) \leq e^{-\frac{\pi c}{12}} e^{2 \pi h} c_{+} \rho_{*}\left(\bar{h}^{\prime}\right) \chi_{0}\left(e^{-2 \pi}\right) .
\end{aligned}
$$


where $\chi_{0}(q)$ is the vacuum character and $q=e^{-\beta}$ where we have assumed that there is no nontrivial conserved current. Here $c_{+}$is defined as

$$
c_{+}=\int_{-\infty}^{\infty} d x \Phi_{+}\left(\bar{h}^{\prime}+\bar{\delta} x\right)
$$

One can extend this argument to a scenario where we have nontrivial conserved currents, then we would have a sum over characters for all the conserved currents

$$
\frac{1}{2 \bar{\delta}} U_{h}(\bar{h}) \leq c_{+} \rho_{*}\left(\bar{h}^{\prime}\right) e^{2 \pi\left(h-\frac{c}{24}\right)} \sum_{\tilde{h}} \chi_{\tilde{h}}\left(e^{-2 \pi}\right) .
$$

This sum over $\tilde{h}$ is convergent as the absolute value of the sum is bounded above by partition function evaluated at $\beta=\bar{\beta}=2 \pi$. Similar result applies to $h, \bar{h}$ getting swapped. Thus for finite $h$ and $\bar{h} \rightarrow \infty$ limit we have

$$
S_{\delta, \bar{\delta}} \leq \mathbb{S}_{h, \delta, \bar{\delta}} \leq 2 \pi \sqrt{\frac{c \bar{h}}{6}}-\frac{3}{4} \log (\bar{h})+2 \pi\left(h+\delta-\frac{c}{24}\right)+\log \left[2 c_{+} \bar{\delta} \sum_{\tilde{h}} \chi_{\tilde{h}}\left(e^{-2 \pi}\right)\right]
$$

where $\chi_{\tilde{h}}$ is the character for the conserved current with weight $(\tilde{h}, 0)$.

The similar result specific for the primaries with finite $h$ and $\bar{h} \rightarrow \infty$ limit, would read:

$$
S_{\delta, \bar{\delta}}^{\mathrm{Vir}} \leq \mathbb{S}_{h, \delta, \bar{\delta}}^{\mathrm{Vir}} \leq 2 \pi \sqrt{\frac{(c-1) \bar{h}}{6}}+2 \pi\left(h+\delta-\frac{c-1}{24}\right)+\log \left[c_{+} \frac{2 \bar{\delta}}{\sqrt{\bar{h}}} \sum_{\tilde{h}} e^{-2 \pi\left(\tilde{h}-\frac{c-1}{24}\right)}\right]
$$

where the zero twist primaries have weight $(\tilde{h}, 0)$. Here again, the sum over $\tilde{h}$ is convergent as the absolute value of the sum is bounded above by partition function evaluated at $\beta=\bar{\beta}=2 \pi$.

CFT without nontrivial zero twist primary. In case the CFT does not have any conserved current, we do not need to worry about $\left(h^{\prime}, 0\right)$ operators anymore and we can do much better as it is possible to choose $\beta \neq 2 \pi$ and still define $\rho_{*}^{\mathrm{ft}-\mathrm{Vir}}\left(h^{\prime}, \bar{h}^{\prime}\right)=$ $\rho_{*}^{\mathrm{ft}-\operatorname{Vir}}\left(h^{\prime}\right) \rho_{*}^{\mathrm{ft}-\mathrm{Vir}}\left(\bar{h}^{\prime}\right)$ as a solution to the following equality:

$$
\int_{0}^{\infty} \mathrm{d} \bar{h}^{\prime} \rho_{*}^{\mathrm{ft}-\operatorname{Vir}}\left(\bar{h}^{\prime}\right) e^{-\bar{\beta}\left(\bar{h}^{\prime}-\frac{c-1}{24}\right)}=\sqrt{\frac{2 \pi}{\bar{\beta}}}\left(1-e^{-\frac{4 \pi^{2}}{\beta}}\right) .
$$

This parallels the analysis for Virasoro primary in section 3 (see (3.41)).

In the present case, using the kernel $\rho_{*}^{\mathrm{ft}-\operatorname{Vir}}\left(h^{\prime}, \bar{h}^{\prime}\right)$ the leading answer turns out to be

$$
\frac{1}{2 \bar{\delta}} U_{h}^{\operatorname{Vir}}(\bar{h}) \leq c_{+} \rho_{*}(\bar{h}) e^{\beta\left(h-\frac{c-1}{24}\right)} e^{\frac{\pi^{2}(c-1)}{6 \beta}} \sqrt{\frac{2 \pi}{\beta}}\left(1-e^{-\frac{4 \pi^{2}}{\beta}}\right) .
$$

Now by appropriately choosing $\beta$ we can recover the "square-root" edge present in the analysis in [7]. The square root edge in $h$ dependence of the density of states should 
produce a factor of $(h-(c-1) / 24)^{3 / 2}$. In particular, we choose $\beta=\frac{1}{\left(h-\frac{c-1}{24}\right)}$ and let $h-\frac{c}{24}$ to be very small ${ }^{8}$

$$
\begin{gathered}
\frac{1}{2 \bar{\delta}} U_{h}^{\operatorname{Vir}}(\bar{h}) \leq c_{+} e \rho_{*}(\bar{h}) e^{\frac{\pi^{2}(c-1)\left(h-\frac{c-1}{24}\right)}{6}} \sqrt{2 \pi\left(h-\frac{c-1}{24}\right)}\left(1-e^{-4 \pi^{2}\left(h-\frac{c-1}{24}\right)}\right) \\
\underset{h-c / 24 \ll \frac{1}{c-1}}{\simeq} c_{+}\left(4 \sqrt{2} \pi^{5 / 2} e\right) \rho_{*}(\bar{h})\left(h-\frac{c-1}{24}\right)^{3 / 2},
\end{gathered}
$$

The above result is consistent with the leading result as reported in $[4,5,7,8]$.

\section{Holographic CFTs}

Holographic CFTs are the ones characterized by a sparse low lying spectrum and large central charge. The sparseness condition is first derived in [19], then rederived in [2], where it emerges naturally out of the Tauberian formalism. In the context of asymptotic behavior of OPE coefficients in large $c$ CFTs, a stronger sparseness condition appears as elucidated in $[29,30]$. In this section we will be exploring such CFTs with large central charge and a low lying sparse spectra. In particular, we derive an expression for the density of states in the limit $h, \bar{h} \sim c \rightarrow \infty$. Following [2], we parameterize $h, \bar{h}$ as

$$
h=c\left(\epsilon+\frac{1}{24}\right), \bar{h}=c\left(\bar{\epsilon}+\frac{1}{24}\right), c \rightarrow \infty, \epsilon-\text { fixed, } \bar{\epsilon}-\text { fixed } .
$$

In this limit the asymptotic of the vacuum crossing kernel is given by

$$
\rho_{*}(h, \bar{h})=\frac{\sqrt{6}}{24} c^{-1}\left(\frac{1}{\epsilon \bar{\epsilon}}\right)^{\frac{3}{4}} e^{2 \pi c\left(\sqrt{\frac{\epsilon}{6}}+\sqrt{\frac{\epsilon}{6}}\right)} \theta(\epsilon) \theta(\bar{\epsilon})+\cdots .
$$

As done in section 3 we separate out the contribution to the partition function into two pieces: the light $Z_{L}$, and the heavy $Z_{H}$. Here we choose

$$
\beta=\frac{\pi}{\sqrt{6 \epsilon}}, \bar{\beta}=\frac{\pi}{\sqrt{6 \bar{\epsilon}}} .
$$

We are required to show that $Z_{H}$ term is sub-leading. $Z_{H}$ term gets contribution whenever $h^{\prime}$ or $\bar{h}^{\prime}$ is greater than $c / 24$ and $\Delta^{\prime}>c / 12$. It can be shown that $Z_{H}$ contribution is sub-leading (the method is exactly similar as in section 3 , one has to be careful about $e^{-\beta c / 24-\bar{\beta} c / 24}$ factor, since $c$ is not finite in the analysis.). The result of the analysis is summarized below:

$$
\Lambda_{ \pm}^{2}<\left(\frac{\sqrt{2} \pi}{\gamma}\right)^{2}\left(1-\frac{\gamma^{2}}{12 \epsilon^{*}}\right)
$$

where $\gamma^{4}=\frac{\epsilon^{*}}{\epsilon_{*}} \geq 1, \epsilon^{*}=\max (\epsilon, \bar{\epsilon}), \epsilon_{*}=\min (\epsilon, \bar{\epsilon})$. The above requires that $1-\frac{\gamma^{2}}{12 \epsilon^{*}}>0$, that is, $\epsilon^{*} \epsilon_{*}=\epsilon \bar{\epsilon}>\frac{1}{12^{2}}$.

\footnotetext{
${ }^{8}$ This is analogous to the condition written down in [7] as $0<\bar{h}-\frac{c-1}{24} \ll 1 / c$, there $\bar{h}$ is finite and $h$ is let to infinity.
} 
In fact, it turns out that we will be requiring much more stronger condition on $\epsilon, \bar{\epsilon}$ :

$$
\begin{aligned}
\epsilon_{*} & >\frac{1}{6}, \epsilon^{*}>\max \left(\frac{\gamma^{4}}{6}, \frac{\gamma^{2}}{12}\right)=\frac{\gamma^{4}}{6}>\frac{1}{6}, \\
\tau & >\frac{5 c}{12} .
\end{aligned}
$$

This condition justifies the assumption that the first term is dominated by the vacuum. We compare this with the result in [19], where the Cardy formula is reported to be applicable for $\epsilon_{*} \epsilon^{*}=\epsilon \bar{\epsilon}>\frac{1}{24^{2}}$. This implies that there is further scope to improve our result and reach the HKS threshold rigorously. If we assume existence of a twist gap $g$, we can push the regime of validity to following:

$$
\epsilon_{*}>\frac{1}{6}\left[\max \left\{\frac{1}{2},\left(1-\frac{6 g}{c}\right)^{2}\right\}\right] .
$$

We will come back to the derivation of the bound on $\epsilon, \bar{\epsilon}$ at the end of this section. For now, with this assumption of vacuum dominance, we find:

$$
e^{-2 \pi c\left(\sqrt{\frac{\epsilon}{6}} \delta+\sqrt{\frac{\epsilon}{6}} \bar{\delta}\right)} \rho_{*}(\epsilon, \bar{\epsilon}) c_{-} \leq \frac{1}{4 \delta \bar{\delta}} \int_{h-\delta}^{h+\delta} \int_{\bar{h}-\bar{\delta}}^{\bar{h}+\bar{\delta}} \mathrm{d} F\left(h, \bar{h}^{\prime}\right) \leq e^{2 \pi c\left(\sqrt{\frac{\epsilon}{6}} \delta+\sqrt{\frac{\epsilon}{6}} \bar{\delta}\right)} \rho_{*}(\epsilon, \bar{\epsilon}) \tilde{c}_{+} .
$$

As a consequence, we find that for fixed $\delta, \bar{\delta}>\delta_{\text {gap }}$,

$$
S_{h, \bar{h}}(\delta, \bar{\delta})=2 \pi \sqrt{\frac{c}{6}\left(h-\frac{c}{24}\right)}+2 \pi \sqrt{\frac{c}{6}\left(\bar{h}-\frac{c}{24}\right)}-\log c+O(1), c \rightarrow \infty .
$$

We can extend the above result to the case where $\delta, \bar{\delta} \sim c^{\alpha}$ where $0<\alpha<1$ by splitting the integral domain into squares of unit area:

$$
\int_{h-\delta}^{h+\delta} \int_{\bar{h}-\bar{\delta}}^{\bar{h}+\bar{\delta}} \mathrm{d} h^{\prime} \mathrm{d} \bar{h}^{\prime} \rho\left(h^{\prime}, \bar{h}^{\prime}\right)=\sum_{m=1}^{2 \delta} \sum_{n=1}^{2 \bar{\delta}} \int_{h-\delta+m-1}^{h-\delta+m} \int_{\bar{h}-\bar{\delta}+n-1}^{\bar{h}-\bar{\delta}+n} \mathrm{~d} h^{\prime} \mathrm{d} \bar{h}^{\prime} \rho\left(h^{\prime}, \bar{h}^{\prime}\right),
$$

and then, using the previous bound, we find

$$
S_{h, \bar{h}}(\delta, \bar{\delta})=2 \pi \sqrt{\frac{c}{6}\left(h+\delta-\frac{c}{24}\right)}+2 \pi \sqrt{\frac{c}{6}\left(\bar{h}+\bar{\delta}-\frac{c}{24}\right)}-\log c+O(1), \quad c \rightarrow \infty .
$$

Now we show that the vacuum contribution dominants the contribution from the light sector. This is straightforward for the sector where $h_{L}<c / 24$ and $\bar{h}_{L}<c / 24$. For this region, we consider the sum

$$
A=e^{\beta h+\bar{\beta} \bar{h}} \sum_{h_{L}, \bar{h}_{L} \leq c / 24} \int \mathrm{d} h^{\prime} \mathrm{d} \bar{h}^{\prime} \rho_{h_{L}, \bar{h}_{L}}\left(h^{\prime}, \bar{h}^{\prime}\right) e^{-\beta h^{\prime}-\bar{\beta} \bar{h}^{\prime}} \Phi_{+}\left(h^{\prime}, \bar{h}^{\prime}\right),
$$

where the crossing kernel of the operator with conformal dimension $\left(h_{L}, \bar{h}_{L}\right)$ is given by

$$
\begin{aligned}
\rho_{h_{L}}(h) & =2 \pi \sqrt{\frac{\frac{c}{24}-h_{L}}{h-\frac{c}{24}}} I_{1}\left(4 \pi \sqrt{\left(\frac{c}{24}-h_{L}\right)\left(h-\frac{c}{24}\right)}\right) \theta\left(h-\frac{c}{24}\right)+\delta\left(h-\frac{c}{24}\right), \\
\rho_{h_{L}, \bar{h}_{L}}(h, \bar{h}) & =\rho_{h_{L}}(h) \rho_{\bar{h}_{L}}(\bar{h}),
\end{aligned}
$$


and the above reproduces the contribution of this operator in the dual channel i.e. at high temperature:

$$
\int d h d \bar{h} \rho_{h_{L}, \bar{h}_{L}}(h, \bar{h}) e^{-\beta h-\bar{\beta} \bar{h}}=e^{-\frac{4 \pi^{2}}{\beta}\left(h_{L}-c / 24\right)-\frac{4 \pi^{2}}{\beta}\left(\bar{h}_{L}-c / 24\right)} .
$$

The asymptotic of the function $\rho_{h_{L}}(h)$ is given by

$$
\rho_{h_{L}}(\epsilon) \sim \frac{1}{2} \frac{1}{6^{1 / 4}} c^{-1 / 2} \epsilon^{-3 / 4}\left(1-\frac{24 h_{L}}{c}\right)^{1 / 4} e^{2 \pi c \sqrt{\frac{\epsilon}{6}\left(1-\frac{24 h_{L}}{c}\right)}} .
$$

Evaluating the each integral by saddle point approximation, we find

$$
A=O\left(c^{-1} e^{2 \pi c \sqrt{\frac{\epsilon}{6}}+2 \pi c \sqrt{\frac{\epsilon}{6}}} \sum_{h_{L}+\bar{h}_{L} \leq \Delta_{H}} e^{-4 \pi \sqrt{6 \epsilon} h_{L}-4 \pi \sqrt{6 \bar{\epsilon}} \bar{h}_{L}}\right) .
$$

Subsequently, using the following sparseness condition

$$
\sum_{h_{L}+\bar{h}_{L} \leq \Delta_{H}} e^{-\beta h_{L}-\bar{\beta} \bar{h}_{L}}=O(1), \beta, \bar{\beta}>2 \pi, c \rightarrow \infty,
$$

we find that

$$
A \sim O\left(\rho_{*}(h, \bar{h})\right)
$$

where the condition $\beta, \bar{\beta}>2 \pi$ translates to $\epsilon, \bar{\epsilon}>1 / 24$. Below we will see that we need to have a more stronger condition i.e. $\epsilon, \bar{\epsilon}>1 / 6$.

We are left to investigate the region where either of the $h_{L}$ or $\bar{h}_{L}$ is greater than $c / 24$. This is basically given by the brown region in figure 15 . Without loss of generality, let us look at the region where $h_{L}>c / 24$. Now the crossing would be given by

$$
\begin{aligned}
\rho_{h_{L}}(h) & =2 \pi \sqrt{-\left(\frac{h_{L}-\frac{c}{24}}{h-\frac{c}{24}}\right)} I_{1}\left(4 \pi \sqrt{-\left(h_{L}-\frac{c}{24}\right)\left(h-\frac{c}{24}\right)}\right) \theta\left(h-\frac{c}{24}\right)+\delta\left(h-\frac{c}{24}\right), \\
\rho_{h_{L}, \bar{h}_{L}}(h, \bar{h}) & =\rho_{h_{L}}(h) \rho_{\bar{h}_{L}}(\bar{h}),
\end{aligned}
$$

We are going to estimate the following:

$$
\begin{aligned}
B & =e^{\beta h+\bar{\beta} \bar{h}} \int \mathrm{d} h^{\prime} \mathrm{d} \bar{h}^{\prime} \rho_{h_{L}, \bar{h}_{L}} e^{-\beta h^{\prime}-\bar{\beta}_{\bar{h}^{\prime}}} \Phi_{+}\left(h^{\prime}, \bar{h}^{\prime}\right) \\
& =e^{\beta(h-c / 24)+\bar{\beta}(\bar{h}-c / 24)} \int \mathrm{d} h^{\prime} \mathrm{d} \bar{h}^{\prime} \rho_{h_{L}, \bar{h}_{L}} e^{-\beta\left(h^{\prime}-c / 24\right)-\bar{\beta}\left(\bar{h}^{\prime}-c / 24\right)} \Phi_{+}\left(h^{\prime}, \bar{h}^{\prime}\right) .
\end{aligned}
$$

The integral over $\bar{h}^{\prime}$ proceeds in usual manner since $\bar{h}_{L}<c / 24$. The integral over $\bar{h}^{\prime}$ proceeds in usual manner since $\bar{h}_{L}<c / 24$. The integral over $h^{\prime}$ requires bit of care. Let 


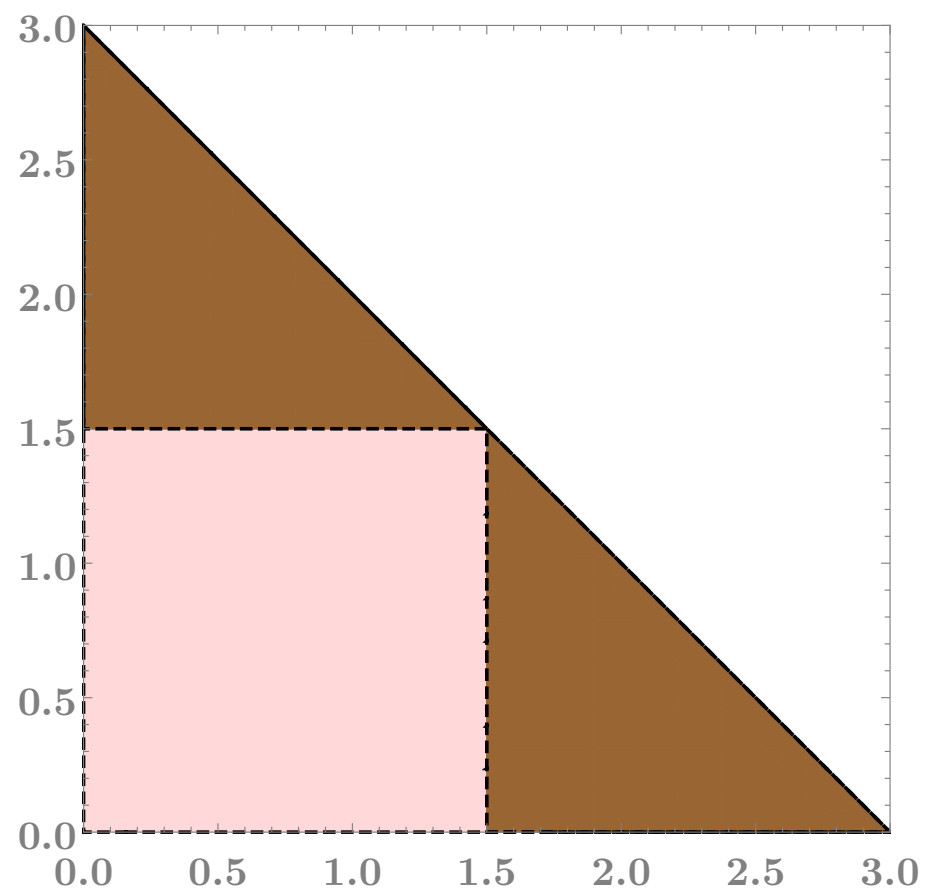

Figure 15. The light region is the union of the region colored with pink and the region colored with brown. The brown regions requires a more careful treatment.

us focus on

$$
\begin{aligned}
& \left|e^{\beta(h-c / 24)} \int \mathrm{d} h^{\prime} \rho_{h_{L}}\left(h^{\prime}\right) e^{-\beta\left(h^{\prime}-c / 24\right)} \Phi_{+}\left(h^{\prime}, \bar{h}^{\prime}\right)\right| \\
& =\left|e^{\beta(h-c / 24)} \int_{c / 24}^{\infty} \mathrm{d} h^{\prime} \rho_{h_{L}}\left(h^{\prime}\right) e^{-\beta\left(h^{\prime}-c / 24\right)} \Phi_{+}\left(h^{\prime}, \bar{h}^{\prime}\right)\right| \\
& =e^{\beta(h-c / 24)} \int_{0}^{\infty} d E^{\prime}\left|\rho_{h_{L}}\left(h^{\prime}\right) e^{-\beta E^{\prime}} \Phi_{+}\left(h^{\prime}, \bar{h}^{\prime}\right)\right| \\
& \leq M e^{\pi c \sqrt{\frac{\epsilon}{6}}} \int d E^{\prime} \frac{1}{\sqrt{E^{\prime}}} e^{-\beta E^{\prime}} \\
& \leq M \sqrt{\left(h_{L}-\frac{c}{24}\right)} \exp \left[\pi c \sqrt{\frac{\epsilon}{6}}\right] \\
& \leq M \sqrt{\frac{1}{c}} \exp \left[2 \pi c \sqrt{\frac{\epsilon}{6}}\right] \exp \left[-2 \pi \sqrt{6 \epsilon} h_{L}\right],
\end{aligned}
$$

where in the second line we have used the $\Theta$ function present in the expression for $\rho_{h_{L}}$. In the penultimate line, we have used $h_{L}<c / 12$. The strict inequality is important to make sure replacing $\sqrt{\left(h_{L}-\frac{c}{24}\right)}$ with $\frac{1}{\sqrt{c}}$ does not spoil the inequality because of the presence of exponential term. Now for the rest of the argument, we will require that

$$
\min (4 \epsilon, \bar{\epsilon})>\frac{1}{6} \& \min (\epsilon, 4 \bar{\epsilon})>\frac{1}{6} \Rightarrow \epsilon, \bar{\epsilon}>\frac{1}{6} .
$$


The above leads to (8.5). Now we sum over all the light states in the brown region to obtain

$$
\begin{aligned}
B & =O\left(\frac{e^{2 \pi c \sqrt{\frac{\epsilon}{6}}+2 \pi c \sqrt{\frac{\epsilon}{6}}}}{c}\left[\sum_{h_{L}>\bar{h}_{L}} e^{-2 \pi \sqrt{6 \epsilon} h_{L}-4 \pi \sqrt{6 \bar{\epsilon}} \bar{h}_{L}}+\sum_{h_{L} \leq \bar{h}_{L}} e^{-4 \pi \sqrt{6 \epsilon} h_{L}-2 \pi \sqrt{6 \bar{\epsilon}} \bar{h}_{L}}\right]\right) \\
& =O\left(\rho_{*}(h, \bar{h})\right) .
\end{aligned}
$$

Twistgap. If we assume a finite twist gap $g$, the (8.21) can be revisited in the light of the twist gap:

$$
\begin{aligned}
& \left|e^{\beta(h-c / 24)} \int \mathrm{d} h^{\prime} \rho_{h_{L}}\left(h^{\prime}\right) e^{-\beta\left(h^{\prime}-c / 24\right)} \Phi_{+}\left(h^{\prime}, \bar{h}^{\prime}\right)\right| \\
& =\left|e^{\beta(h-c / 24)} \int_{c / 24}^{\infty} \mathrm{d} h^{\prime} \rho_{h_{L}}\left(h^{\prime}\right) e^{-\beta\left(h^{\prime}-c / 24\right)} \Phi_{+}\left(h^{\prime}, \bar{h}^{\prime}\right)\right| \\
& =e^{\beta(h-c / 24)} \int_{0}^{\infty} d E^{\prime}\left|\rho_{h_{L}}\left(h^{\prime}\right) e^{-\beta E^{\prime}} \Phi_{+}\left(h^{\prime}, \bar{h}^{\prime}\right)\right| \\
& \leq M e^{\pi c \sqrt{\frac{\epsilon}{6}}} \int d E^{\prime} \frac{1}{\sqrt{E^{\prime}}} e^{-\beta E^{\prime}} \\
& \leq M \sqrt{\left(h_{L}-\frac{c}{24}\right)} \exp \left[\pi c \sqrt{\frac{\epsilon}{6}}\right] \\
& \leq M \sqrt{\frac{1}{c}} \exp \left[2 \pi c \sqrt{\frac{\epsilon}{6}}\right] \exp \left[-2\left(1-\frac{6 g}{c}\right)^{-1} \pi \sqrt{6 \epsilon} h_{L}\right]
\end{aligned}
$$

where now in the penultimate step we use $h_{L} \leq c / 12-g / 2$. And this leads to the modified validity regime as given in (8.7).

\section{Open problems}

We end with a list of open problems which would be nice to figure out:

1. One can hope to use these techniques to investigate the asymptotic OPE coefficients $[6,24,28,30-37]$ and make it spin sensitive. A richer structure in such scenarios is expected as well.

2. For the large spin, finite twist, we have derived an upper bound on the windowed entropy. It would be nice to put a lower bound as well and match up to the results of $[4-8]$. On a conservative note, we remark that even if one can prove a lower bound, it seems hard to distinguish the order one error coming from considering a bin of width $2 \delta$ in spin and the dependence of the extended Cardy formula on finite twist. At present, all our attempts to prove a lower bound provided us with a Cardy like growth multiplied by a negative number, which is trivially true, hence we omitted the details of the trivial lower bound in the text. Furthermore, it would be nice to find out the asymptotic formula for the integrated version (integrated from spin 0 upto some large spin) of the density of states. 
3. It would be nice to have an expression for integrated density of states upto some particular $\Delta$ within a specified range of spin. To be specific, we want to have an estimate of the following quantity:

$$
\int_{0}^{\Delta} \mathrm{d} \Delta^{\prime} \int_{J_{1}}^{J_{2}} \mathrm{~d} J^{\prime} \rho\left(\Delta^{\prime}, J^{\prime}\right), \quad \text { where } J_{1}, J_{2} \in\left[-\Delta^{\prime}, \Delta^{\prime}\right]
$$

4. It would be nice to improve on the value of $r$ and possibly prove that $r=1$ (the parameter appearing in the asymptotic "areal" spectral gap) either by some suitable choice of magic functions or by better estimate of the heavy sector of the partition function. The naive generalization from [3] would not suffice. So one needs to be more creative. And this might shed light on the twist gap and provide a way to expound on the proposed gap in [8].

Our work should be thought of a part of modular bootstrap program [20, 24, 28, 33$35,38-44]$. On a more general ground, it would be interesting to see whether Tauberian theorems and/or Modular bootstrap program can say anything about the chaotic, irrational CFTs. An approach borrowing ideas from Tauberian techniques and that of extremal functionals appearing in [45-51] might be useful in this regard. Furthermore, for holographic CFTs, we can only achieve a reduced regime of validity of Cardy formula compared to what is reported in [19]. It might be possible to improve our result. Albeit, we remark that if the twist gap is greater than $c / 12$, it is possible to achieve the regime of validity of Cardy formula as predicted in [19]. We hope to come back to these problems in future.

\section{Acknowledgments}

The authors acknowledge Ken Intriligator and John McGreevy for fruitful discussions and encouragement. SP thanks Nathan Benjamin, Diptarka Das, Baur Mukhametzhanov, ShuHeng Shao for several illuminating discussions. SP thanks Raghu Mahajan, Ben Michael, Baur Mukhametzhanov for several suggestions regarding improving readability of the draft. SP thanks Simons Center for Geometry and Physics, where a part of this work was presented, for hospitality, and Simeon Hellerman, Zohar Komargodski, Dalimil Mazac for discussions that followed the talk. This work was in part supported by the U.S. Department of Energy (DOE) under cooperative research agreement DE-SC0009919 and Simons Foundation award \#568420. SP also acknowledges the support from Inamori Fellowship, Ambrose Monell Foundation and DOE grant DE-SC0009988.

Open Access. This article is distributed under the terms of the Creative Commons Attribution License (CC-BY 4.0), which permits any use, distribution and reproduction in any medium, provided the original author(s) and source are credited.

\section{References}

[1] J.L. Cardy, Operator Content of Two-Dimensional Conformally Invariant Theories, Nucl. Phys. B 270 (1986) 186 [inSPIRE].

[2] B. Mukhametzhanov and A. Zhiboedov, Modular invariance, tauberian theorems and microcanonical entropy, JHEP 10 (2019) 261 [arXiv: 1904.06359] [INSPIRE]. 
[3] S. Ganguly and S. Pal, Bounds on density of states and spectral gap in $C F T_{2}$, arXiv:1905.12636 [INSPIRE].

[4] Y. Kusuki, Light Cone Bootstrap in General 2D CFTs and Entanglement from Light Cone Singularity, JHEP 01 (2019) 025 [arXiv:1810.01335] [INSPIRE].

[5] Y. Kusuki and M. Miyaji, Entanglement Entropy, OTOC and Bootstrap in 2D CFTs from Regge and Light Cone Limits of Multi-point Conformal Block, JHEP 08 (2019) 063 [arXiv: 1905.02191] [INSPIRE].

[6] S. Collier, Y. Gobeil, H. Maxfield and E. Perlmutter, Quantum Regge Trajectories and the Virasoro Analytic Bootstrap, JHEP 05 (2019) 212 [arXiv:1811.05710] [INSPIRE].

[7] H. Maxfield, Quantum corrections to the BTZ black hole extremality bound from the conformal bootstrap, JHEP 12 (2019) 003 [arXiv: 1906. 04416] [INSPIRE].

[8] N. Benjamin, H. Ooguri, S.-H. Shao and Y. Wang, Light-cone modular bootstrap and pure gravity, Phys. Rev. D 100 (2019) 066029 [arXiv:1906.04184] [INSPIRE].

[9] S. Hellerman, D. Orlando, S. Reffert and M. Watanabe, On the CFT Operator Spectrum at Large Global Charge, JHEP 12 (2015) 071 [arXiv:1505.01537] [INSPIRE].

[10] S. Hellerman, N. Kobayashi, S. Maeda and M. Watanabe, A Note on Inhomogeneous Ground States at Large Global Charge, JHEP 10 (2019) 038 [arXiv:1705.05825] [INSPIRE].

[11] A. Monin, D. Pirtskhalava, R. Rattazzi and F.K. Seibold, Semiclassics, Goldstone Bosons and CFT data, JHEP 06 (2017) 011 [arXiv:1611.02912] [INSPIRE].

[12] G. Cuomo, A. de la Fuente, A. Monin, D. Pirtskhalava and R. Rattazzi, Rotating superfluids and spinning charged operators in conformal field theory, Phys. Rev. D 97 (2018) 045012 [arXiv: 1711.02108] [INSPIRE].

[13] G. Cuomo, Superfluids, vortices and spinning charged operators in $4 d$ CFT, arXiv: 1906.07283 [INSPIRE].

[14] D. Banerjee, S. Chandrasekharan, D. Orlando and S. Reffert, Conformal dimensions in the large charge sectors at the O(4) Wilson-Fisher fixed point, Phys. Rev. Lett. 123 (2019) 051603 [arXiv: 1902.09542] [INSPIRE].

[15] D. Orlando, S. Reffert and F. Sannino, A safe CFT at large charge, JHEP 08 (2019) 164 [arXiv: 1905.00026] [INSPIRE].

[16] S. Favrod, D. Orlando and S. Reffert, The large-charge expansion for Schrödinger systems, JHEP 12 (2018) 052 [arXiv:1809.06371] [INSPIRE].

[17] S.M. Kravec and S. Pal, Nonrelativistic Conformal Field Theories in the Large Charge Sector, JHEP 02 (2019) 008 [arXiv: 1809.08188] [INSPIRE].

[18] S.M. Kravec and S. Pal, The Spinful Large Charge Sector of Non-Relativistic CFTs: From Phonons to Vortex Crystals, JHEP 05 (2019) 194 [arXiv: 1904.05462] [INSPIRE].

[19] T. Hartman, C.A. Keller and B. Stoica, Universal Spectrum of $2 d$ Conformal Field Theory in the Large c Limit, JHEP 09 (2014) 118 [arXiv:1405.5137] [INSPIRE].

[20] S. Collier, Y.-H. Lin and X. Yin, Modular Bootstrap Revisited, JHEP 09 (2018) 061 [arXiv: 1608.06241] [INSPIRE].

[21] L.F. Alday and J.M. Maldacena, Comments on operators with large spin, JHEP 11 (2007) 019 [arXiv:0708.0672] [INSPIRE]. 
[22] D. Pappadopulo, S. Rychkov, J. Espin and R. Rattazzi, OPE Convergence in Conformal Field Theory, Phys. Rev. D 86 (2012) 105043 [arXiv: 1208.6449] [InSPIRE].

[23] J. Qiao and S. Rychkov, A tauberian theorem for the conformal bootstrap, JHEP 12 (2017) 119 [arXiv: 1709.00008] [INSPIRE].

[24] D. Das, S. Datta and S. Pal, Charged structure constants from modularity, JHEP 11 (2017) 183 [arXiv: 1706. 04612] [INSPIRE].

[25] A. Ingham, A tauberian theorem for partitions, Ann. Math. 42 (1941) 1075.

[26] M.A. Subhankulov, Tauberian theorems with remainder term, American Mathematical Society Translations: Series 2, volume 26, Providence RI U.S.A. (1963), pp. 311-338.

[27] B. Mukhametzhanov and A. Zhiboedov, Analytic Euclidean Bootstrap, JHEP 10 (2019) 270 [arXiv: 1808.03212] [INSPIRE].

[28] P. Kraus and A. Maloney, A cardy formula for three-point coefficients or how the black hole got its spots, JHEP 05 (2017) 160 [arXiv:1608.03284] [INSPIRE].

[29] S. Pal, Bound on asymptotics of magnitude of three point coefficients in $2 D$ CFT, arXiv: 1906.11223 [INSPIRE].

[30] B. Michel, Universality in the OPE Coefficients of Holographic 2d CFTs, arXiv:1908.02873 [INSPIRE].

[31] E. Dyer, A.L. Fitzpatrick and Y. Xin, Constraints on Flavored 2d CFT Partition Functions, JHEP 02 (2018) 148 [arXiv: 1709.01533] [INSPIRE].

[32] S. Collier, A. Maloney, H. Maxfield and I. Tsiares, Universal Dynamics of Heavy Operators in $C F T_{2}$, arXiv: 1912.00222 [INSPIRE].

[33] J. Cardy, A. Maloney and H. Maxfield, A new handle on three-point coefficients: OPE asymptotics from genus two modular invariance, JHEP 10 (2017) 136 [arXiv:1705.05855] [INSPIRE].

[34] D. Das, S. Datta and S. Pal, Universal asymptotics of three-point coefficients from elliptic representation of Virasoro blocks, Phys. Rev. D 98 (2018) 101901 [arXiv:1712.01842] [INSPIRE].

[35] E.M. Brehm, D. Das and S. Datta, Probing thermality beyond the diagonal, Phys. Rev. D 98 (2018) 126015 [arXiv:1804.07924] [INSPIRE].

[36] Y. Hikida, Y. Kusuki and T. Takayanagi, Eigenstate thermalization hypothesis and modular invariance of two-dimensional conformal field theories, Phys. Rev. D 98 (2018) 026003 [arXiv: 1804.09658] [INSPIRE].

[37] A. Romero-Bermúdez, P. Sabella-Garnier and K. Schalm, A Cardy formula for off-diagonal three-point coefficients; or, how the geometry behind the horizon gets disentangled, JHEP 09 (2018) 005 [arXiv : 1804.08899] [INSPIRE].

[38] S. Hellerman, A Universal Inequality for CFT and Quantum Gravity, JHEP 08 (2011) 130 [arXiv: 0902.2790] [INSPIRE].

[39] D. Friedan and C.A. Keller, Constraints on 2d CFT partition functions, JHEP 10 (2013) 180 [arXiv: 1307.6562] [INSPIRE].

[40] M. Cho, S. Collier and X. Yin, Genus Two Modular Bootstrap, JHEP 04 (2019) 022 [arXiv: 1705.05865] [INSPIRE]. 
[41] T. Anous, R. Mahajan and E. Shaghoulian, Parity and the modular bootstrap, SciPost Phys. 5 (2018) 022 [arXiv: 1803.04938] [inSPIRE].

[42] N. Afkhami-Jeddi, T. Hartman and A. Tajdini, Fast Conformal Bootstrap and Constraints on 3d Gravity, JHEP 05 (2019) 087 [arXiv: 1903.06272] [INSPIRE].

[43] N. Afkhami-Jeddi, K. Colville, T. Hartman, A. Maloney and E. Perlmutter, Constraints on higher spin $C F T_{2}$, JHEP 05 (2018) 092 [arXiv: 1707.07717] [INSPIRE].

[44] P. Kraus and A. Sivaramakrishnan, Light-state Dominance from the Conformal Bootstrap, JHEP 08 (2019) 013 [arXiv: 1812.02226] [INSPIRE].

[45] D. Mazáč, Analytic bounds and emergence of $A d S_{2}$ physics from the conformal bootstrap, JHEP 04 (2017) 146 [arXiv: 1611.10060] [INSPIRE].

[46] D. Mazáč and M.F. Paulos, The analytic functional bootstrap. Part II. Natural bases for the crossing equation, JHEP 02 (2019) 163 [arXiv:1811.10646] [INSPIRE].

[47] D. Mazáč and M.F. Paulos, The analytic functional bootstrap. Part I. $1 D$ CFTs and $2 D$ S-matrices, JHEP 02 (2019) 162 [arXiv:1803.10233] [INSPIRE].

[48] T. Hartman, D. Mazáč and L. Rastelli, Sphere Packing and Quantum Gravity, JHEP 12 (2019) 048 [arXiv: 1905.01319] [INSPIRE].

[49] D. Mazáč, L. Rastelli and X. Zhou, A Basis of Analytic Functionals for CFTs in General Dimension, arXiv:1910.12855 [INSPIRE].

[50] M.F. Paulos, Analytic Functional Bootstrap for CFTs in $d>1$, arXiv:1910.08563 [INSPIRE].

[51] D. Carmi and S. Caron-Huot, A Conformal Dispersion Relation: Correlations from Absorption, arXiv: 1910.12123 [INSPIRE]. 